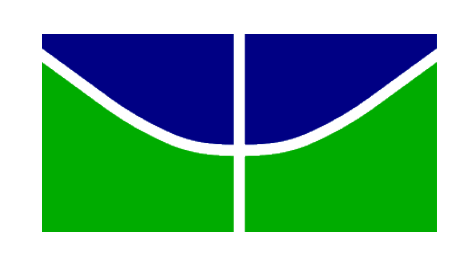

Universidade de Brasília

Instituto de Ciências Exatas

Departamento de Matemática

\title{
Os ideais de uma álgebra associativa gerados por comutadores e tópicos relacionados
}

\author{
por \\ Claud Wagner Gonçalves Dias Júnior \\ Orientador: Prof. Dr. Alexei Krassilnikov
}

Brasília-DF

2016 


\section{Os ideais de uma álgebra associativa gerados por comutadores e tópicos relacionados.} por

\section{Claud Wagner Gonçalves Dias Junior}

Tese apresentada ao Corpo Docente do Programa de Pós-Graduação em Matemática-UnB, como requisito parcial para obtenção do grau de

\section{DOUTOR EM MATEMÁTICA}

Brasília, 25 de novembro de 2016.

\section{Comissão Examinadora:}

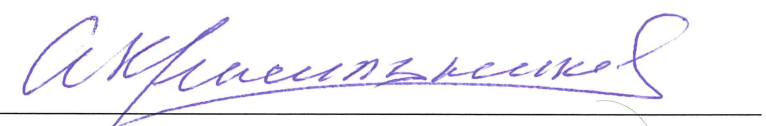

Prof. Dr. Alexei Krassilnikov - Orientador (MAT-UnB)

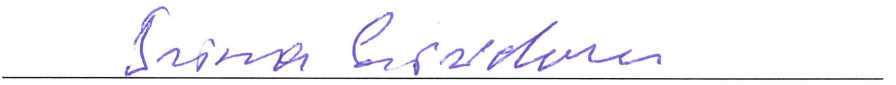

Profa. Dra. Irina Sviridova (MAT-UnB)

$$
\text { Dilla }
$$

Prof. Dr. Victor Petrogradskiy (MAT-UnB)

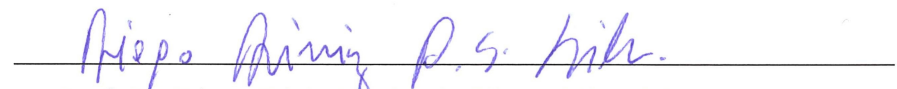

Prof. Dr. Diogo Diniz Pereira da Silva e Silva (UFCG)

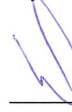

Prof. Dr. Dimas José Gonçalves (UFSCAR) 
Ficha catalográfica elaborada automaticamente, com os dados fornecidos pelo(a) autor(a)

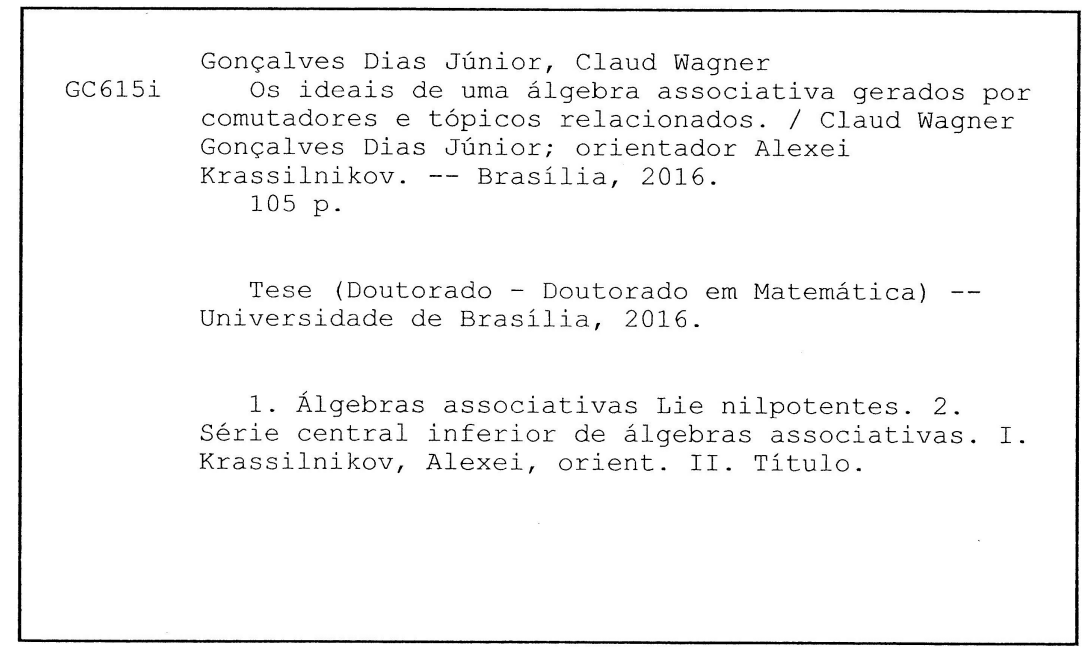




\section{Timoneiro}

(Paulinho da Viola)

Não sou eu quem me navega

Quem me navega é o mar

É ele quem me carrega

Como nem fosse levar.

E quanto mais remo mais rezo

Pra nunca mais se acabar

Essa viagem que faz

0 mar em torno do mar

Meu velho um dia falou

Com seu jeito de avisar:

- Olha, o mar não tem cabelos

Que a gente possa agarrar.

Timoneiro nunca fui

Que eu não sou de velejar

0 leme da minha vida

Deus é quem faz governar

E quando alguém me pergunta

Como se faz pra nadar

Explico que eu não navego

Quem me navega é o mar.

A rede do meu destino

Parece a de um pescador

Quando retorna vazia

Vem carregada de dor

Vivo num redemoinho

Deus bem sabe o que ele faz

A onda que me carrega

Ela mesma é quem me traz 
À minha esposa Aline Ogliari. 


\section{Agradecimentos}

- Ao Grande Arquiteto Universal. Que alegria eu sinto pela bondade de Deus!

- Ao meu guia espiritual, Mestre Gabriel. Que bom poder fazer parte da religião que com tanto sacrifício criaste.

- A minha esposa, Aline Ogliari. Que bom que nos encontramos nessa vida! Lembrese, foi pela sua insistência que eu voltei para a UnB e foi com o seu auxílio que consegui permanecer. Seu amor, sua dedicação, seu carinho e zelo estão nos fundamentos desta conquista.

- A minha mãe, Eva de Fátima. Eu por ti vim ao mundo, abençoado pelo seu amor e contigo aprendi as primeiras lições.

- A todos os meus parentes e familiares e, em especial, minha querida avó Benedita. As melhores lembranças de minha infância.

- A minha filha Araci. Que bom que você chegou na nossa vida! Quando estiver maior e aprender a ler, saiba que enquanto escrevo essas palavras me lembro do seu sorriso e das suas coisas de criança.

- Aos professores da UnB que contribuíram para minha formação e, em especial, os professores Célius Magalhães, Noraí Rocco, Cátia Gonçalves e Mauro Rabelo.

- Aos colegas Silvio Sandro, Raimundo Bastos, Bruno Trindade, Ilana Zuila, Keidna e Jhoel: auxílios valiosos na minha caminhada.

- Ao professor Dimas Gonçalves, que foi meu orientador no mestrado e que me deu as primeiras lições de PI-álgebras. Um dia bati na porta de sua sala (quando ainda era professor na UnB) e perguntei se podia ser meu orientador. Ele disse que trabalhava com PI-álgebras e me perguntou se eu conhecia alguma coisa do assunto. Nunca tinha ouvido falar. Com paciência, me explicou alguma coisa no quadro-negro e, principalmente, me aceitou como aluno. 
- Ao professor Alexei Krassilnikov que aceitou me orientar no doutorado. Esse período de aproximadamente cinco anos foi para mim de uma enorme riqueza. Não só pelo que aprendi do conteúdo, mas principalmente pelo que aprendi vendo a forma com que trabalha a matemática. Ficará registrado em minha memória a imagem de um homem espirituoso, de agradável convivência e de idéias originais. Lembrome de um momento em que eu estava enfrentando uma dificuldade na tese, havia encontrado um resultado e depois descobrimos que ele já existia. Estava perto de completar quatro anos de doutorado e imaginava que a defesa estava próxima. Foi um momento de frustação. Então, o professor Alexei me chamou em sua sala e ligou o computador. Achei que ia me mostrar algum artigo para que eu pudesse me inspirar e resolver o problema. No entanto, ele abriu um vídeo com o Papa Francisco. Perplexo, eu vi uma entrevista em que uma pessoa perguntava ao Papa: o que fazer quando se enfrenta uma dificuldade? O papa respondeu: Primeiro não deseperar nunca, ficar tranquilo e, em seguida, buscar uma maneira de superá-la. E se não for possivel superar, aguardar até que surja a oportunidade de superá-la. Nunca se deve assustar com as dificuldades. Nunca se deve entrar em pânico. Nós somos capazes de superá-las, todas. Precisamos apenas de tempo para compreender, inteligência para buscar o caminho e coragem para seguir em frente. Mas nunca entrar em pânico. Foi melhor do que qualquer artigo. 


\section{Resumo}

Seja $A$ uma álgebra associativa unitária sobre um anel associativo, comutativo e unitário $\mathbb{K}$. Defina o comutador normado à esquerda $\left[a_{1}, a_{2}, \ldots, a_{n}\right]\left(a_{i} \in A\right)$ indutivamente por

$$
\left[a_{1}, a_{2}\right]=a_{1} a_{2}-a_{2} a_{1} ; \quad\left[a_{1}, \ldots, a_{n-1}, a_{n}\right]=\left[\left[a_{1}, \ldots, a_{n-1}\right], a_{n}\right] \quad(n \geq 3)
$$

Para $n \geq 2$, seja $T^{(n)}(A)$ o ideal bilateral de $A$ gerado pelos comutadores $\left[a_{1}, a_{2}, \ldots, a_{n}\right]$ $\left(a_{i} \in A\right)$. Seja $\mathcal{E}=\left\{e_{1}, e_{2}, \ldots\right\}$ um conjunto gerador da álgebra $A$. A primeira parte desta tese diz respeito aos elementos que geram $T^{(n)}(A)$ como um ideal bilateral em $A$. O objetivo principal dessa parte consiste em mostrar que

1. Se $\frac{1}{6} \in \mathbb{K}$, então $T^{(n)}(A)$ é gerado como ideal bilateral pelos comutadores $\left[u_{1}, \ldots, u_{n}\right]$ em que $u_{i} \in \mathcal{E} \cup \mathcal{E}^{2}$

2. Se $\frac{1}{3} \in \mathbb{K}$, então $T^{(n)}(A)$ é gerado como ideal bilateral pelos comutadores $\left[u_{1}, \ldots, u_{n}\right]$ em que $u_{i} \in \mathcal{E} \cup \mathcal{E}^{2} \cup \mathcal{E}^{3}$.

Aqui $\mathcal{E}^{k}(k \geq 1)$ denota o conjunto dos elementos de $A$ da forma $e_{i_{1}} e_{i_{2}} \ldots e_{i_{k}}, e_{i_{j}} \in \mathcal{E}$.

Para isso, em um primeiro momento, será descrito um método recursivo que permite obter um conjunto de geradores para o ideal $T^{(n)}(A)(n \geq 3)$, como ideal bilateral em $A$, a partir dos geradores de $T^{(n-2)}(A)$. A demonstração dos itens 1 e 2 acima é feita com base nesse resultado.

Seja $\mathbb{Z}\langle X\rangle$ a álgebra unitária associativa livre sobre $\mathbb{Z}$ no conjunto $X=\left\{x_{1}, x_{2}, \ldots\right\}$. Considere sua série central inferior como álgebra de Lie, isto é, a série dos ideais de Lie $L^{(i)} \subset \mathbb{Z}\langle X\rangle$ definido recursivamente por $L^{(1)}=\mathbb{Z}\langle X\rangle, L^{(i+1)}=\left[L^{(i)}, \mathbb{Z}\langle X\rangle\right]$, e a correspondente álgebra de Lie graduada associada $B:=\bigoplus_{i \geq 1} B_{i}$, em que $B_{i}=L^{(i)} / L^{(i+1)}$. A segunda parte desta tese diz respeito a série central inferior de $\mathbb{Z}\langle X\rangle$. É bem conhecido que a imagem $\mathcal{J}$ de $T^{(3)}(\mathbb{Z}\langle X\rangle)$ em $B_{1}$ é central na álgebra de Lie $B$. Além disso, sabe-se que o isolador de $\mathcal{J}$ é maior que $\mathcal{J}$. O objetivo principal da segunda parte desta tese é mostrar que o isolador de $\mathcal{J}$ está contido no centro de $B$. 


\section{Abstract}

Let $A$ be an associative unitary algebra over a commutative, associative and unitary ring $\mathbb{K}$. Define a left-normed commutator $\left[a_{1}, a_{2}, \ldots, a_{n}\right]\left(a_{i} \in A\right)$ inductively by

$$
\left[a_{1}, a_{2}\right]=a_{1} a_{2}-a_{2} a_{1} ; \quad\left[a_{1}, \ldots, a_{n-1}, a_{n}\right]=\left[\left[a_{1}, \ldots, a_{n-1}\right], a_{n}\right] \quad(n \geq 3)
$$

For $n \geq 2$, let $T^{(n)}$ be the two-sided ideal in $A$ generated by all commutators $\left[a_{1}, a_{2}, \ldots, a_{n}\right]$ $\left(a_{i} \in A\right)$. Let $\mathcal{E}=\left\{e_{1}, e_{2}, \ldots\right\}$ be a generating set of algebra A. The first part of this thesis concerns with the elements that generate $T^{(n)}(A)$ as two-sided ideal in $A$. The main purpose of the first part of this thesis is to show that

1. If $\frac{1}{6} \in \mathbb{K}$, then $T^{(n)}(A)$ is generated as two-sided ideal by the commutators $\left[u_{1}, \ldots, u_{n}\right]$ where $u_{i} \in \mathcal{E} \cup \mathcal{E}^{2}$

2. If $\frac{1}{3} \in \mathbb{K}$, then $T^{(n)}(A)$ is generated as two-sided ideal by the commutators $\left[u_{1}, \ldots, u_{n}\right]$ where $u_{i} \in \mathcal{E} \cup \mathcal{E}^{2} \cup \mathcal{E}^{3}$.

Here $\mathcal{E}^{k}(k \geq 1)$ denotes the set of elements of the form $e_{i_{1}} e_{i_{2}} \ldots e_{i_{k}}, e_{i_{j}} \in \mathcal{E}$.

For this, at first, we describe a recursive method which allows us to obtain a set of generators for the ideal $T^{(n)}(A)(n \geq 3)$ as a two-sided ideal in $A$ from generators of the ideal $T^{(n-2)}(A)$. The proof of the items 1 and 2 above is based on this result.

Let $\mathbb{Z}\langle X\rangle$ be the free unitary associative algebra over a $\mathbb{Z}$ on the set $X=\left\{x_{1}, x_{2}, \ldots\right\}$. Consider its lower central series as a Lie algebra, i.e., the series of the Lie ideal $L^{(i)} \subset \mathbb{Z}\langle X\rangle$ defined recursively by $L^{(1)}=\mathbb{Z}\langle X\rangle, L^{(i+1)}=\left[L^{(i)}, \mathbb{Z}\langle X\rangle\right]$, and the corresponding associated graded Lie algebra $B:=\bigoplus_{i \geq 1} B_{i}$, where $B_{i}=L^{(i)} / L^{(i+1)}$. The second part of this thesis concerns with the lower central series of $\mathbb{Z}\langle X\rangle$. It is well-known that the image $\mathcal{J}$ of $T^{(3)}(\mathbb{Z}\langle X\rangle)$ in $B_{1}$ is central in the Lie algebra $B$. Furthermore, it is known that the isolator of $\mathcal{J}$ is greater than $\mathcal{I}$. The main purpose of the second part of this thesis is to show that the isolator of $\mathcal{J}$ is contained in the center of $B$. 


\section{Sumário}

1 Introdução 1

1.1 O ideal de uma álgebra associativa gerado por comutadores . . . . . . . . 3

1.2 O centro da álgebra de Lie associada a série central inferior de $\mathbb{Z}\langle X\rangle$. . . 10

2 Conceitos Preliminares $\quad 14$

2.1 Módulos . . . . . . . . . . . . . . . . . . . . . . . . . . . . . 14

2.2 Álgebras . . . . . . . . . . . . . . . . . . . . 24

2.3 Álgebra livre . . . . . . . . . . . . . . . . . . 27

2.4 A base de Specht para $P_{n}$ em $\mathbb{Z}\langle X\rangle \ldots \ldots \ldots \ldots$. . . . . . . . 31

2.5 Fatos relacionados a uma álgebra livre sobre um corpo . . . . . . . . . 36

2.6 A série central inferior de uma álgebra de Lie . . . . . . . . . . . . . . 40

3 Os ideais de uma álgebra associativa gerados por comutadores $\quad 44$

3.1 Resultados auxiliares . . . . . . . . . . . . . . . . . . 45

3.2 Método recursivo para obter os geradores de $T^{(n)} \ldots \ldots \ldots$. . . . . 57

3.3 Os comutadores que geram $T^{(n)}$ como um ideal bilateral . . . . . . . . . . 62

3.4 Resultado principal . . . . . . . . . . . . . . . . . . . . 74

4 O centro da álgebra de Lie associada a série central inferior de $\mathbb{Z}\langle X\rangle \quad 77$

4.1 Uma base para o $\mathbb{Z}$-módulo $T_{n}^{(3)} \ldots \ldots \ldots \ldots \ldots$. . . . . . . . . 79

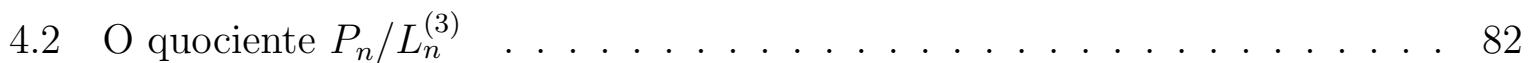

4.3 O quociente $H_{m_{1}, m_{2}, \ldots, m_{k-1}, 1} / H_{m_{1}, m_{2}, \ldots, m_{k-1}, 1} \cap L^{(3)} \ldots \ldots \ldots \ldots$. . . . . 94

4.4 Os elementos do centro de $B$ contidos em $B_{1} \ldots \ldots \ldots$. . . . . . . . . . 99

Referências Bibliográficas 


\section{Capítulo 1}

\section{Introdução}

Neste capítulo, salvo menção contrária, $\mathbb{K}$ é um anel associativo, comutativo e unitário.

Seja $G$ uma $\mathbb{K}$-álgebra de Lie com produto [,] (denominado comutador). O comutador normado à esquerda dos elementos $g_{1}, \ldots, g_{n} \in G(n \geq 3)$ é definido indutivamente como $\left[g_{1}, \ldots, g_{n}\right]=\left[\left[g_{1}, \ldots, g_{n-1}\right], g_{n}\right]$. Para todos $F, H \subset G$, denote por $[F, H]$ o $\mathbb{K}$-submódulo de $G$ gerado pelos elementos $[f, g](f, g \in G)$. Se $F$ e $G$ são ideais de Lie em $G$, então $[F, H]$ é um ideal de Lie em $G$. Defina $L^{(i)}(G), i=1,2, \ldots$, indutivamente por $L^{(1)}(G)=G$ e $L^{(i+1)}(G)=\left[L^{(i)}(G), G\right]$. Assim, obtemos uma série descendente de ideais em $G$ da forma

$$
G=L^{(1)}(G) \supset L^{(2)}(G) \supset L^{(3)}(G) \supset \ldots,
$$

denominada série central inferior da álgebra de Lie $G$. Note que $L^{(i)}(G)$ é o $\mathbb{K}$-submódulo de $G$ gerado por todos os comutadores da forma $\left[g_{1}, \ldots, g_{i}\right]\left(g_{j} \in G\right)$. A álgebra $G$ é dita nilpotente de classe $\leq c$ se $L^{(c+1)}(G)=\{0\}$. Note que se $G$ é nilpotente de classe $\leq c$, então $\left[g_{1}, \ldots, g_{c+1}\right]=0$ para todos $g_{1}, \ldots, g_{c+1} \in G$. A proposição abaixo é bem conhecida e dá uma condição suficiente para que uma álgebra de Lie seja nilpotente.

Proposição 1.1. [28] Seja $G$ uma $\mathbb{K}$-álgebra de Lie gerada por um conjunto $M$. Então $G$ é nilpotente de classe $\leq$ c se, e somente se, $\left[m_{1}, \ldots, m_{c+1}\right]=0$ para todos $m_{1}, \ldots, m_{c} \in M$.

Portanto, para que uma álgebra de Lie seja nilpotente é suficiente que os geradores dela satisfaçam a condição de nilpotência.

Seja $A$ uma $\mathbb{K}$-álgebra associativa e unitária. A álgebra $A$ pode ser vista como uma álgebra de Lie, com a multiplicação de Lie definida por $[a, b]=a b-b a \operatorname{com} a, b \in A$. 
Essa álgebra é denotada por $A^{(-)}$e denominada álgebra de Lie associada a álgebra $A$. Denote o $i$-ésimo elemento da série central inferior da álgebra de Lie $A^{(-)}$por $L^{(i)}(A)$. Temos que $L^{(i)}(A)$ é o submódulo de $A$ gerado por todos os comutadores $\left[a_{1}, \ldots, a_{i}\right]$ $\left(a_{j} \in A\right)$. A álgebra associativa $A$ é dita Lie-nilpotente se $A^{(-)}$é nilpotente, isto é, existe um inteiro $c \geq 1$ tal que $L^{(c+1)}(A)=\{0\}$ ou, equivalentemente, $\left[a_{1}, \ldots, a_{c+1}\right]=0$ para todos $a_{1}, \ldots, a_{c+1} \in A$.

Observando o que foi dito acima (proposição 1.1) para álgebras de Lie nilpotentes, é natural que se coloque a seguinte questão: se $\mathcal{E}$ é um conjunto gerador para a álgebra associativa unitária $A$, então para que $A$ seja Lie-nilpotente de classe $\leq c$ é suficiente que $\left[u_{1}, \ldots, u_{c+1}\right]=0$ para todos $u_{1}, \ldots, u_{c+1} \in \mathcal{E} ?$ Em outras palavras, existe um análogo da proposição 1.1 para álgebras associativas unitárias Lie-nilpotentes? De um modo geral a resposta é negativa. Por exemplo, seja $G$ uma $\mathbb{K}$-álgebra de Lie não abeliana nilpotente ( $\mathbb{K}$ um corpo de característica zero). Existe uma álgebra associativa, denotada por $U(G)$ e denominada álgebra envelopante universal de $G$, tal que $G$ é isomorfa a uma subálgebra de Lie de $U(G)^{(-)}$. Pode-se mostrar que $U(G)$ não é Lie-nilpotente (veja [5], página 201). Nesta tese demonstramos que uma condição suficiente para que a álgebra associativa $A$ seja Lie-nilpotente de classe $\leq c$ é que $\left[u_{1}, \ldots, u_{c+1}\right]=0$ para todo $u_{i} \in A(i=1, \ldots, c+1)$ tal que $u_{i}$ é o produto de, no máximo, 2 elementos de $\mathcal{E}$ quando $\frac{1}{6} \in \mathbb{K}$ ou, no máximo, 3 elementos de $\mathcal{E}$ quando $\frac{1}{3} \in \mathbb{K}$.

Seja $T^{(n)}(A)$ o ideal em $A$ gerado, como ideal bilateral, por todos os comutadores $\left[a_{1}, \ldots, a_{n}\right](n \geq 2)$ em que $a_{i} \in A$. Decorre disso que $T^{(n)}(A)$ é o ideal em $A$ gerado por $L^{(n)}(A)$. Portanto, a álgebra $A$ é Lie-nilpotente de classe $\leq c$ se, e somente, se $T^{(c+1)}(A)=0$. Então, resultados a respeito de $T^{(n)}(A)$ podem ser úteis para saber se a álgebra $A$ é Lie-nilpotente, em especial aqueles que reduzem a lista de geradores dados na definição de $T^{(n)}(A)$. Essa é uma linha que será trabalhada nesta tese.

Um dos primeiros estudos sobre $\mathbb{K}$-álgebras Lie Nilpotentes foi publicado por Jennings (1947, [26]) para $\mathbb{K}=\mathbb{Z}$. Em particular, ele mostrou que se $A$ é uma $\mathbb{Z}$-álgebra associativa Lie-nilpotente então $T^{(2)}(A)$ é um ideal nil enquanto que $T^{(3)}(A)$ é nilpotente. Além disso, se $A$ é finitamente gerado e $A$ é Lie-nilpotente, então $T^{2}(A)$ é nilpotente. Desde então, álgebras associativas Lie-nilpotente tem sido investigadas em vários trabalhos sob diversos 
pontos de vista; veja, por exemplo, [2], [21], [22], [24], [29], [31], [33] e [36].

O recente interesse em álgebras associativas Lie-nilpotentes tem sido motivado pelo estudo dos quocientes $B_{i}(A):=L^{(i)}(A) / L^{(i+1)}(A)(i \geq 1)$ da série central inferior da álgebra de Lie associada a álgebra associativa $A$. O estudo desses quocientes foi iniciado em 2007 no artigo pioneiro de Fiegin e Shoikhet ([17]) para $A=\mathbb{C}\left\langle x_{1}, \ldots, x_{n}\right\rangle$ (a álgebra associativa livre em $n$ geradores sobre o corpo dos complexos $\mathbb{C}$ ). Posteriormente, Dobrovolska et al. ([13], 2008) continuaram o estudo da estrutura de $B_{i}\left(\mathbb{C}\left\langle x_{1}, \ldots, x_{n}\right\rangle\right)$ e, mais geralmente, de $B_{i}(A)$ para uma álgebra associativa $A$ sobre $\mathbb{C}$. Por outro lado, S. Bhupatiraju, P.I. Etingof et al. ([8], 2012) estudaram o caso em que $A=\mathbb{K}\left\langle x_{1}, \ldots, x_{n}\right\rangle$ para $\mathbb{K}=\mathbb{Z}$ e $\mathbb{K}=\mathbb{F}_{p}$. Resultados adicionais sobre este assunto podem ser encontrados, por exemplo, em [1], [3], [4], [6], [10], [14], [16], [25] e [27].

Nesta tese trabalhamos em duas frentes. Uma delas diz respeito ao ideal de uma álgebra associativa gerado por comutadores $\left(T^{(n)}(A)\right)$ e a outra ao centro da álgebra de Lie associada a série central inferior de $\mathbb{Z}\langle X\rangle$ (a álgebra unitária associativa livre sobre $\mathbb{Z}$ no conjunto $\left.X=\left\{x_{1}, x_{2}, \ldots\right\}\right)$. A seguir apresentamos os resultados que foram obtidos em cada uma dessas frentes, bem como um panorama maior do que já se conhece do assunto.

\subsection{O ideal de uma álgebra associativa gerado por comutadores}

Esta seção diz respeito aos resultados que aparecem no capítulo 3 desta tese. Considere como acima que $A$ é uma $\mathbb{K}$-álgebra associativa unitária. Além disso, lembramos que $T^{(n)}(A)$ é o ideal bilateral em $A$ gerado por todos os comutadores $\left[a_{1}, a_{2}, \ldots, a_{n}\right]\left(a_{i} \in A\right)$. Seja $\mathcal{E}=\left\{e_{1}, e_{2}, \ldots\right\}$ um conjunto gerador da álgebra $A$ e denote por $\mathcal{E}^{k}, k \geq 1$, os elementos de $A$ da forma $e_{i_{1}} \ldots e_{i_{k}}\left(e_{i_{j}} \in \mathcal{E}\right)$. Dizemos que um elemento de $\mathcal{E}^{k}$ tem comprimento $k$. Nessas condições, é fácil ver que $T^{(n)}(A)$ é gerado como ideal bilateral em $A$ pelos elementos da forma $\left[a_{1}, \ldots, a_{n}\right]$ em que $a_{i} \in \mathcal{E}^{k_{i}}\left(k_{i}=1,2, \ldots\right)$. O resultado principal do capítulo 3 afirma que, dependendo de $\mathbb{K}$, podemos supor que esses $a_{i}$ 's têm um comprimento máximo. Especificamente, o resultado consiste no seguinte: 


\section{Teorema 1.2 .}

1) Se $\frac{1}{6} \in \mathbb{K}$, então $T^{(n)}(A)$ é gerado como ideal bilateral pelo conjunto

$$
\left\{\left[u_{1}, \ldots, u_{n}\right] \mid u_{i} \in \mathcal{E} \cup \mathcal{E}^{2}\right\}
$$

2) Se $\frac{1}{3} \in \mathbb{K}$, então $T^{(n)}(A)$ é gerado como ideal bilateral pelo conjunto

$$
\left\{\left[u_{1}, \ldots, u_{n}\right] \mid u_{i} \in \mathcal{E} \cup \mathcal{E}^{2} \cup \mathcal{E}^{3}\right\}
$$

Três consequências imediatas desse resultado são dadas abaixo.

Corolário 1.3. Para que a $\mathbb{K}$-álgebra A seja Lie-nilpotente é suficiente que exista um inteiro $c \geq 1$ tal que

- $\left[u_{1}, \ldots, u_{c+1}\right]=0$ para todos $u_{1}, \ldots, u_{c+1} \in \mathcal{E} \cup \mathcal{E}^{2}$ quando $\frac{1}{6} \in \mathbb{K} ;$ ou

- $\left[u_{1}, \ldots, u_{c+1}\right]=0$ para todos $u_{1}, \ldots, u_{c+1} \in \mathcal{E} \cup \mathcal{E}^{2} \cup \mathcal{E}^{3}$ quando $\frac{1}{3} \in \mathbb{K}$

Lembramos que, de acordo com a proposição 1.1, para que uma álgebra de Lie seja nilpotente é suficiente que os geradores dela satisfaçam a condição de nilpotência. Em certo sentido o corolário acima é um análogo dessa proposição para álgebras associativas Lie-nilpotentes.

Corolário 1.4. Seja $\mathbb{K}$ um anel associativo, comutativo e unitário tal que $\frac{1}{3} \in \mathbb{K}$ e seja $B$ uma $\mathbb{K}$-álgebra associativa unitária finitamente gerada. Então $T^{(n)}(B)$ é finitamente gerado como ideal bilateral.

Corolário 1.5. Seja $\mathbb{K}$ um anel associativo, comutativo e unitário tal que $\frac{1}{3} \in \mathbb{K}$ e seja B uma $\mathbb{K}$-álgebra associativa unitária finitamente gerada. Suponha que cada subálgebra de Lie de $B^{(-)}$finitamente gerada seja nilpotente. Então B é Lie-nilpotente.

Ya. P. Sysak ([35]; 2010) propôs a seguinte questão:

Questão 1.6. Seja $R$ um anel associativo. Se $R^{(-)}$é localmente nilpotente, então $R$ é localmente Lie-nilpotente? 
Em outras palavras, a questão pode ser colocada nos seguintes termos: suponha que $R$ é uma $\mathbb{Z}$-álgebra associativa finitamente gerada e que cada subálgebra de Lie de $R^{(-)}$ finitamente gerada seja nilpotente. É verdade que $R$ é Lie-nilpotente?. De acordo com o corolário 1.5 dado acima, no caso de uma $\mathbb{K}$-álgebra associativa unitária em que $\frac{1}{3} \in \mathbb{K}$, a resposta para essa pergunta é afirmativa.

Antes de comentar a linha de raciocínio que permitiu a demonstração do teorema 1.2, vamos examinar o que já se conhece a respeito de geradores para $T^{(n)}(A)$, como ideal bilateral de $A$. O histórico abaixo diz respeito a $T^{(n)}(\mathbb{K}\langle X\rangle)$ em que $\mathbb{K}\langle X\rangle$ é a $\mathbb{K}$-álgebra associativa unitária livre no conjunto não vazio $X$ de geradores livres. Claramente, os resultados apresentados neste histórico são válidos para $T^{(n)}(A)$. Faremos dessa forma, pois é assim que aparecem nos artigos citados. Defina $T^{(n)}(\mathbb{K}\langle X\rangle)=T^{(n)}$. É fácil ver que o ideal $T^{(2)}$ é gerado (como ideal bilateral em $\mathbb{K}\langle X\rangle$ ) pelos polinômios

$$
\left[x_{i_{1}}, x_{i_{2}}\right] \quad\left(x_{j} \in X\right) .
$$

Desta forma, temos um conjunto gerador para $T^{(2)}$ que envolve apenas elementos do conjunto $X$ de geradores livres. Conjuntos geradores similares para $T^{(3)}$ e $T^{(4)}$ (como ideais bilaterais em $\mathbb{K}\langle X\rangle)$ já foram apresentados em alguns trabalhos. Por exemplo, Latyshev ([30], 1963) mostrou que se $\mathbb{K}$ é um corpo de característica zero, então os polinômios

- $\left[x_{i_{1}}, x_{i_{2}}, x_{i_{3}}\right] \quad\left(x_{j} \in X\right)$

- $\left[x_{i_{1}}, x_{i_{2}}\right]\left[x_{i_{3}}, x_{i_{4}}\right]+\left[x_{i_{1}}, x_{i_{3}}\right]\left[x_{i_{2}}, x_{i_{4}}\right] \quad\left(x_{j} \in X\right)$

geram $T^{(3)}$ como ideal bilateral em $\mathbb{K}\langle X\rangle$. Popov ([32], 1979) mostrou que esses polinômios também geram $T^{(3)}$ quando $\mathbb{K}$ é um anel associativo, comutativo e unitário. Esse resultado também pode ser visto no trabalho de Gupta e Krasilnikov ([23], 1999) (que faz referência ao trabalho de Popov).

Etingof, Kim e Ma ([16], 2009) mostraram que, para o corpo dos complexos (característica zero), os polinômios

(i) $\left[x_{i_{1}}, x_{i_{2}}, x_{i_{3}}, x_{i_{4}}\right]$

(ii) $\left[x_{i_{1}}, x_{i_{2}}\right]\left[x_{i_{3}}, x_{i_{4}}, x_{i_{5}}\right]$ 
(iii) $\left[x_{i_{1}}, x_{i_{2}}\right]\left(\left[x_{i_{3}}, x_{i_{4}}\right]\left[x_{i_{5}}, x_{i_{6}}\right]+\left[x_{i_{3}}, x_{i_{5}}\right]\left[x_{i_{4}}, x_{i_{6}}\right]\right) \quad\left(x_{j} \in X\right)$

geram $T^{(4)}$ como ideal bilateral em $\mathbb{K}\langle X\rangle$. No entanto, examinando as demonstrações apresentadas nesse trabalho, nota-se que é suficiente que $\mathbb{K}$ seja um anel associativo, comutativo e unitário tal que $\frac{1}{3} \in \mathbb{K}$. Vale lembrar ainda que esse resultado pode ser deduzido do trabalho de Volichenko ([36], 1978).

Para o caso em que $\mathbb{K}$ é um anel associativo, comutativo e unitário, Deryabina e Krasilnikov ([12], 2013) mostraram que um conjunto de geradores para $T^{(4)}$ consiste dos polinômios dos tipos (i), (iii) e dos polinômios dos tipos

- $\left[x_{i_{1}}, x_{i_{2}}, x_{i_{3}}\right]\left[x_{i_{4}}, x_{i_{5}}, x_{i_{6}}\right] \quad\left(x_{j} \in X\right)$

- $\left[x_{i_{1}}, x_{i_{2}}, x_{i_{3}}\right]\left[x_{i_{4}}, x_{i_{5}}\right]+\left[x_{i_{1}}, x_{i_{2}}, x_{i_{4}}\right]\left[x_{i_{3}}, x_{i_{5}}\right] \quad\left(x_{j} \in X\right)$

- $\left[x_{i_{1}}, x_{i_{2}}, x_{i_{3}}\right]\left[x_{i_{4}}, x_{i_{5}}\right]+\left[x_{i_{1}}, x_{i_{4}}, x_{i_{3}}\right]\left[x_{i_{2}}, x_{i_{5}}\right] \quad\left(x_{j} \in X\right)$.

Recentemente, Costa e Krasilnikov ([11], 2015) mostraram que $T^{(5)}$ é gerado como ideal bilateral de $\mathbb{K}\langle X\rangle$ ( $\mathbb{K}$ associativo, comutativo e unitário) pelos polinômios

- $\left[x_{i_{1}}, x_{i_{2}}, x_{i_{3}}, x_{i_{4}}, x_{i_{5}}\right] \quad\left(x_{j} \in X\right)$,

- $\left[x_{i_{1}}, x_{i_{2}}, x_{i_{3}}\right]\left[x_{i_{4}}, x_{i_{5}}, x_{i_{6}}\right] \quad\left(x_{j} \in X\right)$,

- $\left[x_{i_{1}}, x_{i_{2}}, x_{i_{3}}, x_{i_{4}}\right]\left[x_{i_{5}}, x_{i_{6}}, x_{i_{7}}\right] \quad\left(x_{j} \in X\right)$,

- $\left[x_{i_{1}}, x_{i_{2}}, x_{i_{3}}, x_{i_{4}}\right]\left[x_{i_{5}}, x_{i_{6}}\right]+\left[x_{i_{1}}, x_{i_{2}}, x_{i_{3}}, x_{i_{5}}\right]\left[x_{i_{4}}, x_{i_{6}}\right] \quad\left(x_{j} \in X\right)$,

- $\left[x_{i_{1}}, x_{i_{2}}, x_{i_{3}}\right]\left(\left[x_{i_{4}}, x_{i_{5}}\right]\left[x_{i_{6}}, x_{i_{7}}\right]+\left[x_{i_{4}}, x_{i_{6}}\right]\left[x_{i_{5}}, x_{i_{7}}\right]\right) \quad\left(x_{j} \in X\right)$,

- $\left[\left(\left[x_{i_{1}}, x_{i_{2}}\right]\left[x_{i_{3}}, x_{i_{4}}\right]+\left[x_{i_{1}}, x_{i_{3}}\right]\left[x_{i_{2}}, x_{i_{4}}\right]\right), x_{i_{5}}, x_{i_{6}}\right] \quad\left(x_{j} \in X\right)$,

- $\left[\left(\left[x_{i_{1}}, x_{i_{2}}\right]\left[x_{i_{3}}, x_{i_{4}}\right]+\left[x_{i_{1}}, x_{i_{3}}\right]\left[x_{i_{2}}, x_{i_{4}}\right]\right), x_{i_{5}}\right]\left[x_{i_{6}}, x_{i_{7}}\right]$

$+\left[\left(\left[x_{i_{1}}, x_{i_{2}}\right]\left[x_{i_{3}}, x_{i_{4}}\right]+\left[x_{i_{1}}, x_{i_{3}}\right]\left[x_{i_{2}}, x_{i_{4}}\right]\right), x_{i_{6}}\right]\left[x_{i_{5}}, x_{i_{7}}\right] \quad\left(x_{j} \in X\right)$,

- $\left(\left[x_{i_{1}}, x_{i_{2}}\right]\left[x_{i_{3}}, x_{i_{4}}\right]+\left[x_{i_{1}}, x_{i_{3}}\right]\left[x_{i_{2}}, x_{i_{4}}\right]\right)\left(\left[x_{i_{5}}, x_{i_{6}}\right]\left[x_{i_{7}}, x_{i_{8}}\right]+\left[x_{i_{5}}, x_{i_{7}}\right]\left[x_{i_{6}}, x_{i_{8}}\right]\right) \quad\left(x_{j} \in X\right)$.

Resumindo, conjuntos de geradores para $T^{(n)}(2 \leq n \leq 5)$, como ideal bilateral de $\mathbb{K}\langle X\rangle$, envolvendo apenas elementos do conjunto $X$ de geradores livres já são conhecidos. No capítulo 3 desta tese demonstramos o seguinte resultado: 
Teorema 1.7. Seja $\mathbb{K}$ um anel associativo, comutativo e unitário tal que $\frac{1}{3} \in \mathbb{K}$. Seja $F_{n-2}(n \geq 3)$ um conjunto de polinômios de $\mathbb{K}\langle X\rangle$ que gera, como ideal bilateral, $T^{(n-2)}$. Então $T^{(n)}$ é gerado, como ideal bilateral, pelos polinômios

(1) $\left[f, x_{i_{1}}, x_{i_{2}}\right]$;

(2) $f\left[x_{i_{1}}, x_{i_{2}}, x_{i_{3}}\right]$;

(3) $\left[f, x_{i_{1}}\right]\left[x_{i_{2}}, x_{i_{3}}, x_{i_{4}}\right]$;

(4) $\left[f, x_{i_{1}}\right]\left[x_{i_{2}}, x_{i_{3}}\right]+\left[f, x_{i_{2}}\right]\left[x_{i_{1}}, x_{i_{3}}\right]$;

(5) $f\left(\left[x_{i_{1}}, x_{i_{2}}\right]\left[x_{i_{3}}, x_{i_{4}}\right]+\left[x_{i_{1}}, x_{i_{3}}\right]\left[x_{i_{2}}, x_{i_{4}}\right]\right)$,

em que $f \in F_{n-2}$ e $x_{i_{j}} \in X$.

Note que, por esse teorema, temos um método recursivo que permite obter um conjunto de geradores para o ideal $T^{(n)}(n \geq 3)$, como ideal bilateral de $\mathbb{K}\langle X\rangle$, a partir dos geradores de $T^{(n-2)}$ quando $\frac{1}{3} \in \mathbb{K}$. Por exemplo, vimos que $T^{(2)}$ é gerado como ideal bilateral de $\mathbb{K}\langle X\rangle$ pelos polinômios da forma

$$
\left[x_{i_{1}}, x_{i_{2}}\right] \quad\left(x_{j} \in X\right) .
$$

Assim, pelo teorema 1.7, $T^{(4)}$ é gerado, como ideal bilateral de $\mathbb{K}\langle X\rangle$, pelos polinômios

(iv) $\left[x_{i_{1}}, x_{i_{2}}, x_{i_{3}}, x_{i_{4}}\right]$;

(v) $\left[x_{i_{1}}, x_{i_{2}}\right]\left[x_{i_{3}}, x_{i_{4}}, x_{i_{5}}\right]$;

(vi) $\left[x_{i_{1}}, x_{i_{2}}, x_{i_{3}}\right]\left[x_{i_{4}}, x_{i_{5}}, x_{i_{6}}\right]$;

(vii) $\left[x_{i_{1}}, x_{i_{2}}, x_{i_{3}}\right]\left[x_{i_{4}}, x_{i_{5}}\right]+\left[x_{i_{1}}, x_{i_{2}}, x_{i_{4}}\right]\left[x_{i_{3}}, x_{i_{5}}\right]$;

(viii) $\left[x_{i_{1}}, x_{i_{2}}\right]\left(\left[x_{i_{3}}, x_{i_{4}}\right]\left[x_{i_{5}}, x_{i_{6}}\right]+\left[x_{i_{3}}, x_{i_{5}}\right]\left[x_{i_{4}}, x_{i_{6}}\right]\right)$,

em que $x_{i_{j}} \in X$. Uma vez que os polinômios dos tipos (vi) e (vii) estão no ideal bilateral gerado pelos polinômios (iv), (v) e (viii) (veja o exemplo 3.10 do capítulo 3), então os polinômios dos tipos (iv), (v) e (viii) geram $T^{(4)}$ como ideal bilateral de $\mathbb{K}\langle X\rangle$. Note que 
esse conjunto gerador coincide com o que já é conhecido para $T^{(4)}$ quando $\mathbb{K}$ é um anel associativo, comutativo e unitário tal que $\frac{1}{3} \in \mathbb{K}$, como já foi mencionado.

Usando como conjunto gerador de $T^{(4)}$, como ideal bilateral de $\mathbb{K}\langle X\rangle$, o conjunto formado pelos polinômios dos tipos (iv), (v) e (viii) temos, pelo teorema 1.7, que $T^{(6)}$ é gerado, como ideal bilateral de $\mathbb{K}\langle X\rangle$, pelos polinômios

- $\left[x_{i_{1}}, x_{i_{2}}, x_{i_{3}}, x_{i_{4}}, x_{i_{5}}, x_{i_{6}}\right]$;

- $\left[x_{i_{1}}, x_{i_{2}}, x_{i_{3}}, x_{i_{4}}\right]\left[x_{i_{5}}, x_{i_{6}}, x_{i_{7}}\right]$;

- $\left[x_{i_{1}}, x_{i_{2}}, x_{i_{3}}, x_{i_{4}}, x_{i_{5}}\right]\left[x_{i_{6}}, x_{i_{7}}, x_{i_{8}}\right]$;

- $\left[x_{i_{1}}, x_{i_{2}}, x_{i_{3}}, x_{i_{4}}, x_{i_{5}}\right]\left[x_{i_{6}}, x_{i_{7}}\right]+\left[x_{i_{1}}, x_{i_{2}}, x_{i_{3}}, x_{i_{4}}, x_{i_{6}}\right]\left[x_{i_{5}}, x_{i_{7}}\right]$;

- $\left[x_{i_{1}}, x_{i_{2}}, x_{i_{3}}, x_{i_{4}}\right]\left(\left[x_{i_{5}}, x_{i_{6}}\right]\left[x_{i_{7}}, x_{i_{8}}\right]+\left[x_{i_{5}}, x_{i_{7}}\right]\left[x_{i_{6}}, x_{i_{8}}\right]\right)$;

- $\left[\left[x_{i_{1}}, x_{i_{2}}\right]\left[x_{i_{3}}, x_{i_{4}}, x_{i_{5}}\right], x_{i_{6}}, x_{i_{7}}\right]$;

- $\left[x_{i_{1}}, x_{i_{2}}\right]\left[x_{i_{3}}, x_{i_{4}}, x_{i_{5}}\right]\left[x_{i_{6}}, x_{i_{7}}, x_{i_{8}}\right]$;

- $\left[\left[x_{i_{1}}, x_{i_{2}}\right]\left[x_{i_{3}}, x_{i_{4}}, x_{i_{5}}\right], x_{i_{6}}\right]\left[x_{i_{7}}, x_{i_{8}}, x_{i_{9}}\right]$;

- $\left[\left[x_{i_{1}}, x_{i_{2}}\right]\left[x_{i_{3}}, x_{i_{4}}, x_{i_{5}}\right], x_{i_{6}}\right]\left[x_{i_{7}}, x_{i_{8}}\right]+\left[\left[x_{i_{1}}, x_{i_{2}}\right]\left[x_{i_{3}}, x_{i_{4}}, x_{i_{5}}\right], x_{i_{7}}\right]\left[x_{i_{6}}, x_{i_{8}}\right]$;

- $\left[x_{i_{1}}, x_{i_{2}}\right]\left[x_{i_{3}}, x_{i_{4}}, x_{i_{5}}\right]\left(\left[x_{i_{6}}, x_{i_{7}}\right]\left[x_{i_{8}}, x_{i_{9}}\right]+\left[x_{i_{6}}, x_{i_{8}}\right]\left[x_{i_{7}}, x_{i_{9}}\right]\right)$;

- $\left[\left[x_{i_{1}}, x_{i_{2}}\right]\left(\left[x_{i_{3}}, x_{i_{4}}\right]\left[x_{i_{5}}, x_{i_{6}}\right]+\left[x_{i_{3}}, x_{i_{5}}\right]\left[x_{i_{4}}, x_{i_{6}}\right]\right), x_{i_{7}}, x_{i_{8}}\right]$;

- $\left[x_{i_{1}}, x_{i_{2}}\right]\left(\left[x_{i_{3}}, x_{i_{4}}\right]\left[x_{i_{5}}, x_{i_{6}}\right]+\left[x_{i_{3}}, x_{i_{5}}\right]\left[x_{i_{4}}, x_{i_{6}}\right]\right)\left[x_{i_{7}}, x_{i_{8}}, x_{i_{9}}\right]$;

- $\left[\left[x_{i_{1}}, x_{i_{2}}\right]\left(\left[x_{i_{3}}, x_{i_{4}}\right]\left[x_{i_{5}}, x_{i_{6}}\right]+\left[x_{i_{3}}, x_{i_{5}}\right]\left[x_{i_{4}}, x_{i_{6}}\right]\right), x_{i_{7}}\right]\left[x_{i_{8}}, x_{i_{9}}, x_{i_{10}}\right]$;

- $\left[\left[x_{i_{1}}, x_{i_{2}}\right]\left(\left[x_{i_{3}}, x_{i_{4}}\right]\left[x_{i_{5}}, x_{i_{6}}\right]+\left[x_{i_{3}}, x_{i_{5}}\right]\left[x_{i_{4}}, x_{i_{6}}\right]\right), x_{i_{7}}\right]\left[x_{i_{8}}, x_{i_{9}}\right]$

$$
+\left[\left[x_{i_{1}}, x_{i_{2}}\right]\left(\left[x_{i_{3}}, x_{i_{4}}\right]\left[x_{i_{5}}, x_{i_{6}}\right]+\left[x_{i_{3}}, x_{i_{5}}\right]\left[x_{i_{4}}, x_{i_{6}}\right]\right), x_{i_{8}}\right]\left[x_{i_{7}}, x_{i_{9}}\right]
$$

- $\left[x_{i_{1}}, x_{i_{2}}\right]\left(\left[x_{i_{3}}, x_{i_{4}}\right]\left[x_{i_{5}}, x_{i_{6}}\right]+\left[x_{i_{3}}, x_{i_{5}}\right]\left[x_{i_{4}}, x_{i_{6}}\right]\right)\left(\left[x_{i_{7}}, x_{i_{8}}\right]\left[x_{i_{9}}, x_{i_{10}}\right]+\left[x_{i_{7}}, x_{i_{9}}\right]\left[x_{i_{8}}, x_{i_{10}}\right]\right)$, 
em que $x_{i_{j}} \in X$.

Os exemplos dados acima mostram como o teorema 1.7 pode ser utilizado para obter recursivamente geradores para os ideais $T^{(n)}$ quando $n$ é par. No capítulo 3 serão dados exemplos para $n$ ímpar. No exemplo 3.12 veremos que usando o teorema 1.7 e eliminando os polinômios que são consequência dos demais conseguimos um conjunto gerador para $T^{(3)}$, como ideal bilateral de $\mathbb{K}\langle X\rangle$, que coincide com o que já é conhecido para esse ideal.

O capítulo 3 está dividido em quatro seções. A primeira delas tem como objetivo a demonstração do seguinte lema:

Lema 1.8. Sejam $S$ um subconjunto (não vazio) de $\mathbb{K}\langle X\rangle$, U o ideal bilateral de $\mathbb{K}\langle X\rangle$ gerado, como ideal bilateral, por $S$ e $W$ o ideal bilateral de $\mathbb{K}\langle X\rangle$ gerado, como ideal bilateral, pelos elementos da forma $\left[u, a_{1}, a_{2}\right]$ em que $u \in U$ e $a_{1}, a_{2} \in \mathbb{K}\langle X\rangle$. Então $W$ coincide com o ideal bilateral de $\mathbb{K}\langle X\rangle$ gerado, como ideal bilateral, pelos polinômios

(1) $\left[s, x_{i_{1}}, x_{i_{2}}\right]$;

(2) $s\left[x_{i_{1}}, x_{i_{2}}, x_{i_{3}}\right]$;

(3) $\left[s, x_{i_{1}}\right]\left[x_{i_{2}}, x_{i_{3}}, x_{i_{4}}\right]$;

(4) $\left[s, x_{i_{1}}\right]\left[x_{i_{2}}, x_{i_{3}}\right]+\left[s, x_{i_{2}}\right]\left[x_{i_{1}}, x_{i_{3}}\right]$;

(5) $s\left(\left[x_{i_{1}}, x_{i_{2}}\right]\left[x_{i_{3}}, x_{i_{4}}\right]+\left[x_{i_{1}}, x_{i_{3}}\right]\left[x_{i_{2}}, x_{i_{4}}\right]\right)$, em que $x_{i_{j}} \in X$ e $s \in S$.

Cabe observar que esse lema é um dos resultados desse capítulo. Na segunda seção é feita a demonstração do teorema 1.7 ("o método recursivo"). Ela está fundamentada em dois resultados: o lema 1.8 mencionado acima e um resultado conhecido que é dado abaixo.

Lema 1.9. [22] Se $\frac{1}{3} \in \mathbb{K}$, então $T^{(n)}=\mathbb{K}\langle X\rangle\left[T^{(n-2)}, \mathbb{K}\langle X\rangle, \mathbb{K}\langle X\rangle\right]$ para todo $n \geq 3$. Também são apresentados nessa seção casos particulares do teorema 1.7, conforme foi comentado anteriormente. Na terceira seção é feita a demonstração do teorema 1.2 ("do comprimento máximo") para $\mathbb{K}\langle X\rangle$. Ela é feita por indução sendo fundamental o uso do teorema 1.7 ("o método recursivo") na etapa indutiva. Por fim, na quarta seção é feita a demonstração do resultado principal (teorema 1.2) bem como de suas conseqûencias. 


\subsection{O centro da álgebra de Lie associada a série cen- tral inferior de $\mathbb{Z}\langle X\rangle$}

Esta seção diz respeito aos resultados que aparecem no capítulo 4 desta tese. Como antes, $A$ é uma $\mathbb{K}$-álgebra associativa unitária. A série central inferior de $A^{(-)}$(a álgebra $A$ vista como álgebra de Lie) é definida recursivamente por $L^{(1)}(A)=A, L^{(i+1)}(A)=\left[L^{(i)}(A), A\right]$ $(i \geq 1)$.

Considere os quocientes

$$
B_{i}(A):=L^{(i)}(A) / L^{(i+1)}(A), \quad i \geq 1
$$

e a soma direta deles, isto é, $B(A):=\bigoplus_{i \geq 1} B_{i}(A)$. Dados $a \in L^{(i)}(A)$ e $b \in L^{(j)}(A)$, definimos em $B(A)$ o produto [, ] tal que $\left[a+L^{(i+1)}(A), b+L^{(j+1)}(A)\right]:=[a, b]+L^{(i+j+1)}(A)$. Desta forma, $B(A)$ é uma álgebra de Lie graduada, pois $\left[B_{i}(A), B_{j}(A)\right] \subset B_{i+j}(A)$. Além disso, $B(A)$ é gerada como álgebra de Lie por $B_{1}(A)$.

O estudo de $B_{i}(A)$ foi iniciado com o trabalho de B. Feigin e B. Shoikhet ([17], 2006), que consideraram o caso em que $A=\mathbb{C}\left\langle x_{1}, \ldots, x_{n}\right\rangle$, a álgebra associativa livre em $n$ geradores sobre o corpo dos complexos $\mathbb{C}$. Uma observação importante de Feigin e Shoikhet nesse trabalho é que a imagem de $T^{(3)}(A)$ em $B_{1}(A)$ está contida no centro de $B(A)$. Denote por $\bar{B}_{1}(A)$ o quociente de $B_{1}(A)$ por essa imagem. Além disso, eles mostraram que $B_{i}(A)(i>1)$ são representações da álgebra de Lie $W_{n}$ do campo de vetores polinomiais em $n$ variáveis e determinaram a exata estrutura de $\bar{B}_{1}(A)$ e $B_{2}(A)$ como representações de $W_{n}$.

Posteriormente, Dobrovolska et al. ([13], 2008) continuaram o estudo da estrutura de $B_{i}\left(\mathbb{C}\left\langle x_{1}, \ldots, x_{n}\right\rangle\right)$ e, mais geralmente, de $B_{i}(A)$ para uma álgebra associtiva $A$ sobre $\mathbb{C}$. Por exemplo, eles construiram uma base para $B_{2}\left(\mathbb{C}\left\langle x_{1}, \ldots, x_{n}\right\rangle\right)$ e determinaram as estruturas de $B_{3}\left(\mathbb{C}\left\langle x_{1}, x_{2}\right\rangle\right)$ e $B_{4}\left(\mathbb{C}\left\langle x_{1}, x_{2}\right\rangle\right)$, confirmando conjecturas de [17]. Por outro lado, S. Bhupatiraju, P.I. Etingof et al. ([8], 2012) estudaram o caso em que $A=\mathbb{K}\left\langle x_{1}, \ldots, x_{n}\right\rangle$ para $\mathbb{K}=\mathbb{Z}$ e $\mathbb{K}=\mathbb{F}_{p}$. Segue abaixo alguns dos resultados apresentados em [8]. 
- ([8], proposição 3.9) A torção em

$$
\bar{B}_{1}\left(\mathbb{Z}\left\langle x_{1}, \ldots, x_{n}\right\rangle\right)\left(m_{1}, \ldots, m_{n}\right)
$$

é isomorfa a $\left(\mathbb{Z} / m d c\left(m_{1}, \ldots, m_{n}\right)\right)^{2^{n-2}}$.

$\left(\left(m_{1}, \ldots, m_{n}\right)\right.$ denota a parte de multigrau $\left.m_{1}, \ldots, m_{n}\right)$

- ([8], corolário 3.10) A torção em

$$
\bar{B}_{1}\left(\mathbb{Z}\left\langle x_{1}, x_{2}\right\rangle\right)\left(m_{1}, m_{2}\right)
$$

é isomorfa a $\mathbb{Z} / m d c\left(m_{1}, m_{2}\right)$ e é gerada pelo elemento $x_{1}^{m_{1}-1} x_{2}^{m_{2}-1}\left[x_{1}, x_{2}\right]$.

- ([8], teorema 4.1) Seja $w=\left[x_{3}, x_{3}^{m_{3}-1} x_{1}^{m_{1}-1} x_{2}^{m_{2}-1}\left[x_{1}, x_{2}\right]\right]$. Então

$$
w+L^{(3)}\left(\mathbb{Z}\left\langle x_{1}, x_{2}, x_{3}\right\rangle\right)
$$

é um elemento de torção de $B_{2}\left(\mathbb{Z}\left\langle x_{1}, x_{2}, x_{3}\right\rangle\right)$ de ordem dividindo $m d c\left(m_{1}, m_{2}, m_{3}\right)$; Em particular, se $m d c\left(m_{1}, m_{2}, m_{3}\right)=2$ ou 3 , então a ordem de $w+L^{(3)}\left(\mathbb{Z}\left\langle x_{1}, x_{2}, x_{3}\right\rangle\right)$ é igual a $m d c\left(m_{1}, m_{2}, m_{3}\right)$.

- ([8], teorema 4.3) $B_{2}\left(\mathbb{Z}\left\langle x_{1}, x_{2}\right\rangle\right)$ é livre de torção.

- ([8], teorema 4.8) A torção em $B_{2}\left(\mathbb{Z}\left\langle x_{1}, \ldots, x_{n}\right\rangle\right), n \geq 3$, é isomorfa a um quociente $\operatorname{de}\left(\mathbb{Z} / m d c\left(m_{1}, \ldots, m_{n}\right)\right)^{2^{n-2}-1}$.

Consta também nesse trabalho tabelas com dados experimentais (obtidos com recurso computacional: pacote MAGMA) de elementos de torção e, em particular, para o quociente $B_{3}\left(\mathbb{Z}\left\langle x_{1}, x_{2}, x_{3}\right\rangle\right)$ os autores afirmam existir elementos de torção de ordem 2 e 3. Resultados adicionais sobre este assunto podem ser encontrados, por exemplo, em [1], [3], [4], [6], [10], [14], [16], [25] e [27].

Conforme mencionamos acima, Feigin e Shoikhet em [17] mostraram que a imagem de $T^{(3)}(A)$ em $B_{1}(A)$ está contida no centro de $B(A)$ quando $A=\mathbb{C}\left\langle x_{1}, \ldots, x_{n}\right\rangle$ em que $\mathbb{K}$ é um corpo de característica zero. No entanto, S. Bhupatiraju, P.I. Etingof et al em [8] observaram que esse fato é verdadeiro quando $\mathbb{K}$ é um anel associativo, comutativo e unitário. Considere $\mathbb{Z}\langle X\rangle$, a $\mathbb{Z}$-álgebra associativa livre num conjunto não vazio $X$ de 
geradores livres, e defina $L^{(i)}=L^{(i)}(\mathbb{Z}\langle X\rangle), B_{i}:=B_{i}(\mathbb{Z}\langle X\rangle)$ e $B=B(\mathbb{Z}\langle X\rangle)$. Denote por $\mathcal{J}$ a imagem de $T^{(3)}(\mathbb{Z}\langle X\rangle)$ em $B_{1}$ e defina $\bar{B}_{1}:=B_{1} / \mathcal{J}$. Então $\bar{B}:=B / \mathcal{J}=\bar{B}_{1} \oplus B_{2} \oplus \ldots$ é uma álgebra de Lie graduada. Essas considerações foram feitas em [8]. Para a sequência do texto, será dada a definição de isolador.

Definição 1.10. Seja $M$ um $\mathbb{K}$-módulo. Se $N$ é um $\mathbb{K}$-submódulo de $M$, o isolador de $N$ é o conjunto

$$
I(N)=\{m \in M \mid \exists k \in \mathbb{K}, k \neq 0, \text { tal que } k m \in N\} .
$$

No caso em que $\mathbb{K}$ é um domínio, $I(N)$ é um $\mathbb{K}$-submódulo de $M$ que contém $N$. É fácil ver que $M / N$ tem torção se, e somente se, o isolador de $N$ contém propriamente $N$ (isto é, $I(N)$ é maior que $N)$. De acordo com a proposição 3.9 de [8] (mencionada acima) $\bar{B}_{1}=B_{1} / \mathcal{J}$ tem torção e, portanto, o isolador de $\mathcal{J}$ é maior que $\mathcal{J}$. O resultado principal do capítulo 4 consiste no seguinte:

Teorema 1.11. O isolador de $\mathcal{J}$ está contido no centro de B.

Desta forma, definimos a álgebra de Lie graduada $\widetilde{B}:=B / I(\mathcal{J})=B_{1} / I(\mathcal{J}) \oplus B_{2}(A) \oplus \ldots$ A demonstração desse teorema tem como peça chave o seguinte resultado:

Teorema 1.12. $H_{m_{1}, \ldots, m_{k-1}, 1} / H_{m_{1}, \ldots, m_{k-1}, 1} \cap L^{(3)}$ é um $\mathbb{Z}$-módulo livre e, portanto, não tem torção.

Sendo que $H_{m_{1}, \ldots, m_{k-1}, 1}$ é o $\mathbb{Z}$-submódulo dos polinômios multi-homogêneos em $\mathbb{Z}\langle X\rangle$ com multigrau $\left(m_{1}, \ldots, m_{k-1}, 1\right)$.

Cabe observar que o fato do $\mathbb{Z}$-módulo $H_{m_{1}, \ldots, m_{k-1}, 1} / H_{m_{1}, \ldots, m_{k-1}, 1} \cap L^{(3)}$ não ter torção pode ser extraído do teorema 4.8 de [8]. No entanto, os argumentos usados aqui seguem outro caminho. C. Bekh-Ochir e D. M. Riley em [7] mostraram que em $\mathbb{K}\langle X\rangle$, $\mathbb{K}$ um corpo de característica zero, é válida a decomposição dos espaços vetoriais

$$
T^{(3)} \cap P_{n}=\left(L^{(3)} \cap P_{n}\right) \bigoplus\left(T^{(3)} \cap P_{n-1}\right) x_{n}
$$

em que $P_{n}$ é o $\mathbb{K}$-submódulo de $\mathbb{K}\langle X\rangle$ gerado pelos polinômios multilineares em $x_{1}, \ldots, x_{n}$. Nesse artigo, os autores utilizaram, em partes essenciais das demonstrações, argumentos 
de dimensão que são próprios de espaços vetoriais. Mostramos que essa decomposição é válida no contexto de $\mathbb{Z}$-módulos (veja o teorema 4.25 do capítulo 4 ). É por esse caminho que provamos o teorema 1.12 .

O capítulo 4 está dividido em quatro seções. Na primeira delas é dada uma base linear para $T^{(3)}$. O ponto de partida é a descrição de uma certa base (denominada base de Specht) para $P_{n}$ (em $\left.\mathbb{Z}\langle X\rangle\right)$. Deve-se observar que é um resultado bem conhecido (veja [34]). Além disso, a base obtida para $T^{(3)}$ é a mesma que aparece em [7]. A diferença é que em [7] essa base é dada em $\mathbb{K}\langle X\rangle$ quando $\mathbb{K}$ é um corpo de característica zero.

Na segunda seção, mostramos que em $\mathbb{Z}\langle X\rangle$ é válida a decomposição dada em 1.1. Na terceira seção é feita a demonstração do teorema 1.12. Por fim, na quarta seção é dada a demonstração do resultado principal (teorema 1.11). 


\section{Capítulo 2}

\section{Conceitos Preliminares}

Este capítulo é uma coletânea de resultados conhecidos que serão utilizados no decorrer desta tese. Ele foi escrito com base nos livros [5], [15],[18] e [20].

\subsection{Módulos}

Definição 2.1. Seja $\mathbb{K}$ um anel associativo, comutativo e unitário. Um grupo aditivo $(M,+)$ dotado de uma multiplicação por escalar

$$
\begin{aligned}
\mathbb{K} \times M & \longrightarrow M \\
(k, m) & \longmapsto k \cdot m
\end{aligned}
$$

é dito um $\mathbb{K}$-módulo à esquerda ou um módulo à esquerda sobre $\mathbb{K}$ se satisfaz os seguintes axiomas $\left(\forall k_{1}, k_{2} \in \mathbb{K}\right.$ e $\left.\forall m_{1}, m_{2} \in M\right)$ :

I) $1 \cdot m_{1}=m_{1}$;

II) $\left(k_{1} k_{2}\right) \cdot m_{1}=k_{1} \cdot\left(k_{2} \cdot m_{1}\right)$;

III) $\left(k_{1}+k_{2}\right) \cdot m_{1}=k_{1} \cdot m_{1}+k_{2} \cdot m_{1}$;

IV) $k_{1} \cdot\left(m_{1}+m_{2}\right)=k_{1} \cdot m_{1}+k_{1} \cdot m_{2}$.

Se $k \in \mathbb{K}$ e $m \in M$, escreveremos também $k m$ para denotar o elemento $k \cdot m$. Analogamente, define-se $\mathbb{K}$-módulo à direita. No que segue, estudaremos sempre módulos à esquerda sobre anéis. E, quando não houver perigo de confusão, usaremos simplesmente 
a expressão $\mathbb{K}$-módulo. Note que se o anel $\mathbb{K}$ for um corpo então o $\mathbb{K}$-módulo $M$ é um espaço vetorial.

Proposição 2.2. Seja $M$ um $\mathbb{K}$-módulo. Então

(i) $0 m=0, \forall m \in M$;

(ii) $k 0=0, \forall k \in \mathbb{K}$;

(iii) $(-k) m=k(-m)=-(k m), \forall k \in \mathbb{K}, \forall m \in M$.

Demonstração. (i) e (ii) Basta notar que

$$
0(m)=(0+0) m=0 m+0 m \quad \text { e } \quad k 0=k(0+0)=k 0+k 0 .
$$

iii) Temos

$$
0=0 m=(k+(-k)) m=k m+(-k) m
$$

e, portanto, $(-k) m=-(k m)$. Temos também,

$$
0=k 0=k(m+(-m))=k m+k(-m)
$$

e, portanto, $k(-m)=-(k m)$. Segue então o resultado.

Como consequência da proposição acima, temos $(-1)(-m)=1 m=m$ para todo elemento $m$ de um $\mathbb{K}$-módulo.

Exemplo 2.3. Todo ideal (à esquerda) I de um anel $A$ é um A-módulo para a operação $A \times I \longrightarrow I$ dada pela multiplicação em A: se $a \in A$ e $b \in I$ então ab $\in I$. Em particular, todo anel $A$ é um A-módulo.

Para distinguir o anel $\mathbb{K}$ do mesmo conjunto considerado como $\mathbb{K}$-módulo usamos o símbolo $\mathbb{K} \mathbb{K}$.

Exemplo 2.4. Todo grupo abeliano $G$ pode ser visto como um $\mathbb{Z}$-módulo. De fato, dados $g \in G$ e $n \in \mathbb{Z}$, definimos $n g$ da seguinte forma:

- Se $n=0$, então $0 g:=0$.

- Se $n \geq 1$, então $n g:=(n-1) g+g$. Assim, $1 g=g, 2 g=g+g, 3 g=g+g+g$, etc. 
- Se $n<0$, então $n g:=|n|(-g)$. Assim $-1 g=1 .(-g)=-g,-2 g=2(-g)=$ $(-g)+(-g)$, etc.

Suponha que um grupo abeliano $G$ seja um $\mathbb{Z}$-módulo. Sejam $n \in \mathbb{Z}$ e $g \in G$. Se $n>0$, então

$$
n g=((n-1)+1) g=(n-1) g+g .
$$

Se $n<0$, então

$$
n g=n((-1)(-g))=(n(-1))(-g)=(-n)(-g)=|n|(-g) \text {. }
$$

Desta forma, todo $\mathbb{Z}$-módulo se comporta como no exemplo 2.4 .

Definição 2.5. Seja $\left\{M_{\alpha}\right\}_{\alpha \in \Gamma}$ uma família arbitrária de $\mathbb{K}$-módulos. Definimos sua soma direta $\bigoplus_{\alpha \in \Gamma} M_{\alpha}$ como sendo o conjunto:

$\left\{\right.$ aplicações $m: \Gamma \longrightarrow \bigcup_{\alpha \in \Gamma} M_{\alpha} \mid m(\alpha) \in M_{\alpha}(\forall \alpha \in \Gamma), m(\alpha)=0$ para quase todo $\left.\alpha \in \Gamma\right\}$ e, se $m \in \bigoplus_{\alpha \in \Gamma} M_{\alpha}$ escreveremos $m(\alpha)=m_{\alpha}$.

Na definição acima, a frase " $m(\alpha)=0$ para quase todo $\alpha \in \Gamma$ " deve ser entendida como: o conjunto $\{\alpha \in \Gamma \mid m(\alpha) \neq 0\}$ é finito.

Munido das operações:

- $\left(m_{1}+m_{2}\right)(\alpha)=m_{1}(\alpha)+m_{2}(\alpha) \forall \alpha \in \Gamma$,

- $(k m)(\alpha)=k m(\alpha) \forall \alpha \in \Gamma, k \in \mathbb{K}$,

damos a $\bigoplus_{\alpha \in \Gamma} M_{\alpha}$ uma estrutura de $\mathbb{K}$-módulo.

Definição 2.6. Seja $M$ um $\mathbb{K}$-módulo. Um subgrupo $N$ de $M$ é um $\mathbb{K}$-submódulo se a multiplicação escalar de $M$ preserva $N$, isto é, se

$$
k m \in N, \quad \forall k \in \mathbb{K} \quad e \forall m \in N .
$$

A proposição abaixo é uma consequência imediata da definição acima. 
Proposição 2.7. Seja $M$ um $\mathbb{K}$-módulo. Um subconjunto não vazio $N$ de $M$ é um $\mathbb{K}$ submódulo de $M$ se, e somente se $\left(k_{1} m_{1}+k_{2} m_{2}\right) \in N$ para quaisquer $m_{1}, m_{2} \in N$ e $k_{1}, k_{2} \in \mathbb{K}$

Seja $M$ um $\mathbb{K}$-módulo e $N$ um $\mathbb{K}$-submódulo de $M$. Considerando apenas a estrutura de grupo aditivo de $M$ podemos construir o grupo quociente $M / N=\{m+N \mid m \in M\}$. Sobre este grupo $(M / N,+)$ podemos considerar a seguinte multiplicação por escalar:

$$
\begin{aligned}
& \mathbb{K} \times M / N \longrightarrow M / N \\
&(k, m+N) \longmapsto \\
& k m+N
\end{aligned}
$$

É fácil ver que esta operação é bem definida e que $M / N$ é um $\mathbb{K}$-módulo, denominado $\mathbb{K}$-módulo quociente de $M$ por $N$.

Definição 2.8. Sejam $M$ e $N$ dois $\mathbb{K}$-módulos. Uma aplicação $\phi: M \longrightarrow N$ é um homomorfismo de $\mathbb{K}$-módulos ou um $\mathbb{K}$-homomorfismo se

i) $\phi\left(m_{1}+m_{2}\right)=\phi\left(m_{1}\right)+\phi\left(m_{2}\right), \quad \forall m_{1}, m_{2} \in M$;

ii) $\phi(k m)=k \phi(m), \quad \forall k \in \mathbb{K} e \forall m \in M$.

Definição 2.9. Sejam $M$ e $N$ dois $\mathbb{K}$-módulos e $\phi$ um $\mathbb{K}$-homomorfismo de $M$ em $N$ tal que $\phi$ é bijetivo. Dizemos então que $\phi$ é um $\mathbb{K}$-isomorfismo de $M$ em $N$.

Quando existir um $\mathbb{K}$-isomorfismo entre dois $\mathbb{K}$-módulos $M$ e $N$, dizemos que eles são isomorfos e escrevemos $M \cong N$.

Seja $M$ um $\mathbb{K}$-módulo. Denote por $\operatorname{End}_{\mathbb{K}}(M)$ o conjunto dos $\mathbb{K}$-endomorfismos de $M$, isto é, homomorfismos de $M$ em $M$. Dados $f, g \in \operatorname{End}_{\mathbb{K}}(M)$, a aplicação $f+g: M \longrightarrow M$ definida por $(f+g)(m):=f(m)+g(m)$, para todo $m \in M$, é um $\mathbb{K}$-endomorfismo de $M$. Além disso, $f \circ g \in \operatorname{End}_{\mathbb{K}}(M)$. É fácil ver que o conjunto $\operatorname{End}_{\mathbb{K}}(M)$ munido das operações

$$
\begin{aligned}
+: \operatorname{End}_{\mathbb{K}}(M) \times \operatorname{End}_{\mathbb{K}}(M) & \longrightarrow \operatorname{End}_{\mathbb{K}}(M) \\
(f, g) & \longmapsto f+g
\end{aligned}
$$

e

$$
\begin{aligned}
\cdot: \operatorname{End}_{\mathbb{K}}(M) \times \operatorname{End}_{\mathbb{K}}(M) & \longrightarrow \operatorname{End}_{\mathbb{K}}(M) \\
(f, g) & \longmapsto f \circ g
\end{aligned}
$$

é um anel associativo e unitário. 
Definição 2.10. Seja $M$ um $\mathbb{K}$-módulo. Um conjunto $B \subset M$ é dito uma base para $M$ se todo elemento de $M$ pode ser escrito unicamente como uma combinação linear finita de elementos de B com coeficientes em $\mathbb{K}$. Equivalentemente:

i) Dado $m \in M$, existem $k_{1}, \ldots, k_{n} \in \mathbb{K} e b_{1}, \ldots, b_{n} \in B$ tais que $m=k_{1} b_{1}+\ldots+k_{n} b_{n}$. Em outras palavras, $B$ gera $M$;

ii) Dados $k_{1}, \ldots, k_{n} \in \mathbb{K}$ e $b_{1}, \ldots, b_{n} \in M$, se $k_{1} b_{1}+\ldots+k_{n} b_{n}=0$, então $k_{i}=0$ para $i=1, \ldots, n$. Em outras palavras, $B$ é um conjunto linearmente independente (l.i.).

Definição 2.11. Um $\mathbb{K}$-módulo $M$ é dito um $\mathbb{K}$-módulo livre se ele possui uma base.

Exemplo 2.12. Todo espaço vetorial sobre um corpo $\mathbb{K}$ é um $\mathbb{K}$-módulo livre.

Exemplo 2.13. Se $\mathbb{K}$ é um anel unitário, o $\mathbb{K}$-módulo $\mathbb{K}_{\mathbb{K}} \mathbb{K}$ é livre e o conjunto $\left\{1_{\mathbb{K}}\right\}$ é uma base.

Agora vamos ver uma importante caracterização dos módulos livres.

Proposição 2.14. Seja $M$ um $\mathbb{K}$-módulo. Então $M$ é um $\mathbb{K}$-módulo livre se, e somente se $M$ é isomorfo a uma soma direta de cópias de $\mathbb{K}$.

Demonstração. ( $\Longrightarrow$ ) Se $M$ é um módulo livre, considere $B=\left\{b_{\alpha}\right\}_{\alpha \in \Gamma}$ uma base de $M$. Então, dado um elemento $m \in M$, podemos escrevê-lo de forma única como $m=\sum_{\alpha \in \Gamma} k_{\alpha} b_{\alpha}$, com $k_{\alpha} \in \mathbb{K}, \forall \alpha \in \Gamma$ e $k_{\alpha}=0$ para quase todo $\alpha \in \Gamma$. Dessa forma, defina:

$$
\phi: M \longrightarrow \bigoplus_{\alpha \in \Gamma} \mathbb{K}, \quad m=\sum_{\alpha \in \Gamma} k_{\alpha} b_{\alpha} \longmapsto s \text { em que } s_{\alpha}=k_{\alpha}, \quad \forall \alpha \in \Gamma
$$

É fácil ver $\phi$ é um isomorfismo de $\mathbb{K}$-módulos.

$(\Longleftarrow)$ Se $M \cong \bigoplus_{\alpha \in \Gamma} \mathbb{K}$, seja $\phi: M \longrightarrow \bigoplus_{\alpha \in \Gamma} \mathbb{K}$ um isomorfismo de $\mathbb{K}$-módulos. Considere o conjunto $B=\left\{e_{\beta} \in \bigoplus_{\alpha \in \Gamma} \mathbb{K} \mid \beta \in \Gamma\right\}$ dado por:

$$
e_{\beta}(i)=\left\{\begin{array}{lll}
1 & \text { se } & i=\beta \\
0 & \text { se } & i \in \Gamma \backslash\{\beta\}
\end{array}\right.
$$

Note que $B$ é uma base para $\bigoplus_{\alpha \in \Gamma} \mathbb{K}$. De fato, 
- Seja $s \in \bigoplus_{\alpha \in \Gamma} \mathbb{K}$. Então $s: \Gamma \longrightarrow \bigcup_{\alpha \in \Gamma} \mathbb{K}$ é tal que $s(\alpha) \in \mathbb{K} \forall \alpha \in \Gamma$ e $s(\alpha)=0$ para quase todo $\alpha \in \Gamma$. Assim

$$
s(i)=\sum_{\alpha \in \Gamma} s(\alpha) e_{\alpha}(i), \text { para } i \in \Gamma,
$$

e, portanto, $B$ gera $\bigoplus_{\alpha \in \Gamma} \mathbb{K}$.

- Se $k_{\beta_{1}} e_{\beta_{1}}+\ldots+k_{\beta_{n}} e_{\beta_{n}}=0$, em que $k_{\beta_{i}} \in \mathbb{K}$, então

$$
\left(k_{\beta_{1}} e_{\beta_{1}}+\ldots+k_{\beta_{n}} e_{\beta_{n}}\right)\left(\beta_{i}\right)=0, \quad \forall i=1, \ldots, n
$$

Logo, $k_{\beta_{i}}=0, \forall i=1, \ldots, n$ e, portanto, o conjunto $B$ é l.i.

Uma vez que isomorfismos preservam bases, temos que o conjunto $\left\{\phi^{-1}\left(e_{\beta}\right) \mid \beta \in \Gamma\right\}$ é uma base para $M$.

Corolário 2.15. Seja $M$ um $\mathbb{K}$-módulo livre. Então $M=\{0\}$ ou $\# M \geq \# \mathbb{K}$.

Demonstração. Pela proposição 2.14, se $M$ é livre, então $M \cong \bigoplus_{\alpha \in \Gamma} \mathbb{K}$. Assim, se $M \neq\{0\}$, então $\Gamma \neq \emptyset$ e, portanto, $\# M=\# \bigoplus_{\alpha \in \Gamma} \mathbb{K} \geq \# \mathbb{K}$.

Segue do corolário acima que se $M$ é um $\mathbb{Z}$-módulo livre então $M$ não pode ser finito. Em particular, o $\mathbb{Z}$-módulo $\mathbb{Z}_{n}$ não é um $\mathbb{Z}$-módulo livre.

Se $A$ é um anel arbitrário, então duas bases distintas de um $A$-módulo livre $M$ têm a mesma cardinalidade? Quando $A$ é um corpo e, portanto $M$ é um espaço vetorial, sabemos que a resposta é afirmativa. No entanto, existem módulos livres com bases de diferentes cardinalidades, como mostra o exemplo abaixo.

Exemplo 2.16. Suponha que $M$ é um $\mathbb{K}$-módulo livre com base $\left\{b_{1}, b_{2}, \ldots\right\}$. Seja $A=$ $\operatorname{End}_{\mathbb{K}}(M)$ o anel dos endomorfismos de $M$. Como todo anel, A é um módulo livre sobre si mesmo e $\left\{1_{A}\right\}$ é uma base. Sejam $\varphi_{1}, \varphi_{2}$ endomorfismos de $M$ determinados por:

$$
\varphi_{1}\left(b_{i}\right)= \begin{cases}b_{(i+1) / 2} & \text { se } i \text { é ímpar } \\ 0 & \text { se } i \text { é par }\end{cases}
$$

e

$$
\varphi_{2}\left(b_{i}\right)=\left\{\begin{array}{lll}
0 & \text { se } & i \text { é impar } \\
b_{i / 2} & \text { se } & i \text { é par. }
\end{array}\right.
$$


O A-módulo A também é livre com base $\left\{\varphi_{1}, \varphi_{2}\right\}$. Para ver isso, note primeiro que para todos $g_{1}, g_{2} \in A$, o endomorfismo $h=g_{1} \varphi_{1}+g_{2} \varphi_{2}$ satisfaz

$$
h\left(b_{2 i}\right)=g_{2}\left(b_{i}\right) \text { e } h\left(b_{2 i-1}\right)=g_{1}\left(b_{i}\right),
$$

para todo $i \geq 1$. Por isso, se $h$ é o endomorfismo nulo, então $g_{1}$ e $g_{2}$ devem ser nulos. Assim o conjunto $\left\{\varphi_{1}, \varphi_{2}\right\}$ é linearmente independente.

Por outro lado, dado qualquer $f \in A$, sejam $f_{1}, f_{2}$ endomorfismos de $M$ determinados por

$$
f_{1}\left(b_{i}\right)=f\left(b_{2 i-1}\right) \text { e } f_{2}\left(b_{i}\right)=f\left(b_{2 i}\right)
$$

para todo $i \geq 1$. Disso segue imediatamente que $f=f_{1} \varphi_{1}+f_{2} \varphi_{2}$. Por isso, $\left\{\varphi_{1}, \varphi_{2}\right\}$ gera A.

Definição 2.17. Um anel $A$ possui a propriedade de invariância dimensional se, para qualquer A-módulo livre $M$, todas as bases de $M$ possuem a mesma cardinalidade. Nesse caso, ao cardinal comum das bases de $M$ chama-se dimensão de $M$, e escreve-se $\operatorname{dim}_{A} M$. Esse cardinal comum também é denominado de posto de $M$.

O anel $A$ definido no exemplo 2.16 não tem a propriedade de invariância dimensional. No entanto, módulos sobre uma grande classe de anéis (que incluem, por exemplo, anéis comutativos não nulos) têm a propriedade de invariância dimensional.

Uma vez que módulos são generalizações de espaços vetoriais, é natural que alguns resultados clássicos de espaços vetoriais não sejam verdadeiros para módulos em geral. Espaços vetoriais são módulos livres em que todas as bases têm o mesmo posto. Aí já temos uma diferença na comparação com módulos. Segue abaixo uma sequência de exemplos que ilustram outras diferenças entre espaços vetoriais e módulos.

Exemplo 2.18. Em geral não é verdade que todo subconjunto linearmente independente de um módulo livre possa ser ampliado a uma base.

De fato, o módulo $\mathbb{Z} \mathbb{Z}$ é livre e o conjunto 2 é linearmente independente. No entanto não é base e nem pode ser ampliado a uma base, visto que $\mathbb{Z} \mathbb{Z}$ tem posto 1 .

Exemplo 2.19. É falso, em geral, que todo conjunto gerador contém uma base.

Uma vez que todo inteiro $n$ pode ser escrito como $n=3 n-2 n$, segue que o $\{2,3\}$ é um conjunto gerador de $\mathbb{Z} \mathbb{Z}$. No entanto, esse conjunto não contém nenhuma base. 
Exemplo 2.20. Nem sempre um submódulo de um módulo livre é livre.

Considere o $\mathbb{Z}_{6}$-submódulo $H=\{\overline{0}, \overline{2}, \overline{4}\}$ de $\mathbb{Z}_{6} \mathbb{Z}_{6}$. Uma vez que todos os elementos de $H$ são linearmente dependentes, temos que $H$ não é livre. No entanto, $\mathbb{Z}_{6} \mathbb{Z}_{6}$ é livre.

Exemplo 2.21. Seja $M$ um A-módulo livre e $S \subsetneq M$ um submódulo, também livre. Nem sempre é verdade que o número de elementos de uma base de $S$ é menor que o número de elementos de uma base de $M$.

Considere o $\mathbb{Z}$-módulo $\mathbb{Z} \oplus \mathbb{Z}$ e o submódulo $S$ gerado pelos elementos linearmente independentes $(1,1)$ e $(-1,1)$. Temos que o conjunto $\{(1,0),(0,1)\}$ é uma base de $\mathbb{Z} \oplus \mathbb{Z}$ e que $(1,0),(0,1) \notin S$. Assim, $S \subsetneq \mathbb{Z} \oplus \mathbb{Z}$ e $\operatorname{dim}_{\mathbb{Z}} S=\operatorname{dim}_{\mathbb{Z}} \mathbb{Z} \oplus \mathbb{Z}$.

A proposição abaixo nos dá uma condição sobre um anel $\mathbb{K}$ afim de que os submódulos de um $\mathbb{K}$-módulo livre também sejam livres.

Proposição 2.22. Seja $M$ um $\mathbb{K}$-módulo livre, em que $\mathbb{K}$ é um domínio de ideais principais (DIP), e seja $N$ um $\mathbb{K}$-submódulo de $M$. Então $N$ também é livre e o posto de $N$ é menor ou igual ao posto de $M$.

Demonstração. Seja $B=\left\{b_{i} \mid i \in I\right\}$ uma base para $M$. Pelo axioma da escolha existe uma relação de ordem $\leq$ para o conjunto $B$ de forma que $(B, \leq)$ é bem ordenado. Desta forma, todo elemento não nulo $u \in N$ pode ser escrito como

$$
u=k_{\alpha} b_{\alpha}+k_{\beta} b_{\beta}+\cdots+k_{\gamma} b_{\gamma}
$$

em que $b_{\alpha}>b_{\beta}>\ldots>b_{\gamma}$ e $k_{\alpha}$ é um elemento não nulo de $\mathbb{K}$. Denote por $\lambda(u)$ o termo líder dessa combinação. Assim $\lambda(u)=k_{\alpha} b_{\alpha}$.

Seja $A=\{a \in B \mid \exists k \in \mathbb{K}$ tal que $k a=\lambda(u)$ para algum $u \in N\}$. Para cada $a \in A$ defina $J(a)=\{0\} \cup\{k \in \mathbb{K} \mid k a=\lambda(u)$ para algum $u \in N\}$. Temos que $J(a)$ é um ideal de $\mathbb{K}$. De fato, sejam $r, r^{\prime} \in J(a)$ e $k \in \mathbb{K}$. Assim, $r a=\lambda(u)$ e $r^{\prime} a=\lambda\left(u^{\prime}\right)$ em que $u, u^{\prime} \in N$. Se $r+r^{\prime} \neq 0$ então $\left(r+r^{\prime}\right) a=\lambda\left(u+u^{\prime}\right)$ e, portanto, $r+r^{\prime} \in J(a)$. Se $k r \neq 0$, então $k r a=\lambda(k u)$ e, portanto $k r \in J(a)$. Seja $n_{a} \in J(a)$ o gerador desse ideal. Então existe um $u \in N$ tal que $\lambda(u)=n_{a} a$. Seja $u(a)$ um determinado elemento de $N$ com essa propriedade (aqui usamos novamente o axioma da escolha). Afirmamos que $C=\{u(a) \mid a \in A\}$ é uma base para $N$. 
De fato, sejam $u\left(a_{1}\right), \ldots, u\left(a_{t}\right)$ elementos distintos de $C$ e suponha que $a_{1}>\ldots>a_{t}$. Temos que $u\left(a_{i}\right)=n_{a_{i}} a_{i}+v\left(a_{i}\right)$, em que $i=1, \ldots, t$ e $v\left(a_{i}\right)$ é uma combinação linear de elementos de $B$ menores que $a_{i}$. Note que $a_{i}$ não pode aparecer em nenhum $v\left(a_{j}\right)$ em que $j>i$, pois do contrário $a_{i}<a_{j}$. Seja

$$
0=\sum_{i=1}^{t} k_{i} u\left(a_{i}\right)=\sum_{i=1}^{t}\left(k_{i} n_{a_{i}} a_{i}+k_{i} v\left(a_{i}\right)\right)
$$

em que $k_{i} \in \mathbb{K}$. Como $a_{1}$ aparece uma única vez nessa última expressão, temos que $k_{1}=0$ e a expressão se reduz a $\sum_{i=2}^{t}\left(k_{i} n_{a_{i}} a_{i}+k_{i} v\left(a_{i}\right)\right)$. Mas $a_{2}$ aparece uma única vez nessa expressão e, portanto $k_{2}=0$. Prosseguindo com esse raciocínio temos que os elementos de $C$ são linearmente independentes.

Resta agora mostrar que os elementos de $C$ geram $N$. Suponha que não. Tome então $u \in N$ tal que $u$ não é uma combinação linear de elementos de $C$ de modo que $\lambda(u)=k a$ e $a \in B$ é minimal. Desta forma, $a \in A$ e $k \in J(a)=\left(n_{a}\right)$. Então $k=r n_{a}$ e $\lambda(r u(a))=r n_{a} a=k a$. Consequentemente, $u-r u(a)$ que está em $N$ e não é combinação linear de elementos de $C$ tem termo líder menor que $a$ contrariando a minimalidade de $a$.

Finalmente, note que a base $C$ de $N$ tem a mesma cardinalidade do subconjunto $A$ de $B$ e, portanto o posto de $N$ é menor ou igual ao posto de $M$.

A recíproca da proposição acima também é válida:

Proposição 2.23. Seja $\mathbb{K}$ um domínio de integridade tal que para todo o $\mathbb{K}$-módulo livre $M$ os submódulos $N$ de $M$ são livres. Então $\mathbb{K}$ é um domínio de ideais principais (DIP).

Demonstração. Uma vez que $\mathbb{K} \mathbb{K}$ é um $\mathbb{K}$-módulo livre, temos, por hipótese, que todos os $\mathbb{K}$-submódulos de $\mathbb{K} \mathbb{K}$ são livres. Seja $I$ um ideal de $\mathbb{K}$. Claramente, $I$ é um $\mathbb{K}$-submódulo de $\mathbb{K} \mathbb{K}$. Note que uma base de $I$ só pode conter um elemento pois quaisquer dois elementos $a, b \in I$ são linearmente dependentes:

$$
(-b) a+a b=0
$$

Finalmente, se $\{d\}$ é uma base de $I$, em particular $I$ é gerado por $d$, logo $I$ é principal.

Definição 2.24. Seja $M$ um $\mathbb{K}$-módulo. Um elemento $m \in M, m \neq 0$, tal que $k m=0$, para algum $k \in \mathbb{K}, k \neq 0$, é chamado de elemento de torção de $M$. 
Seja $M$ um $\mathbb{K}$-módulo. Denote por

$$
\operatorname{Tor}(M):=\{m \in M \mid \exists k \in \mathbb{K} \backslash\{0\} \text { tal que } k m=0\} .
$$

Em outras palavras Tor $(M)$ é o conjunto formado pelos elementos de torção de $M$ e pelo 0 . Se $M=\operatorname{Tor}(M)$ diz-se que $M$ é um módulo de torção; se $\operatorname{Tor}(M)=\{0\}$ diz que $M$ é livre de torção.

Exemplo 2.25. O $\mathbb{Z}$-módulo $\mathbb{Z}_{n}, n \geq 2$, é de torção enquanto que o $\mathbb{Z}_{2}$-módulo $\mathbb{Z}_{2}$ é livre de torção.

Proposição 2.26. Seja $M$ um $\mathbb{K}$-módulo em que $\mathbb{K}$ é um domínio de integridade. Então o Tor $(M)$ é um $\mathbb{K}$-submódulo de $M$.

Demonstração. Sejam $m_{1}, m_{2} \in \operatorname{Tor}(M)$ e $d_{1}, d_{2} \in \mathbb{K}$. Então existem $k_{1}, k_{2} \in \mathbb{K}$, não nulos, tais que $k_{1} m_{1}=0$ e $k_{2} m_{2}=0$. Logo,

$$
\begin{gathered}
\left(k_{1} k_{2}\right)\left(d_{1} m_{1}+d_{2} m_{2}\right)=\left(k_{1} k_{2}\right)\left(d_{1} m_{1}\right)+\left(k_{1} k_{2}\right)\left(d_{2} m_{2}\right)=\left(k_{1} k_{2} d_{1}\right) m_{1}+\left(k_{1} k_{2} d_{2}\right) m_{2} \\
=\left(k_{2} d_{1} k_{1}\right) m_{1}+\left(k_{1} d_{2} k_{2}\right) m_{2}=\left(k_{2} d_{1}\right)\left(k_{1} m_{1}\right)+\left(k_{1} d_{2}\right)\left(k_{2} m_{2}\right)=0,
\end{gathered}
$$

e uma vez que $k_{1} k_{2} \neq 0$ ( $\mathbb{K}$ é um domínio), então $\left(d_{1} m_{1}+d_{2} m_{2}\right) \in \operatorname{Tor}(M)$.

Proposição 2.27. Se $M$ é um $\mathbb{K}$-módulo livre, em que $\mathbb{K}$ é um domínio de integridade, então $\operatorname{Tor}(M)=0$.

Demonstração. Seja $\left\{e_{i}\right\}_{i \in I}$ uma base para $M$ e $m \in \operatorname{Tor}(M)$ então, por um lado existem $k_{1}, \ldots, k_{n} \in \mathbb{K}$ tais que $m=k_{1} e_{i_{1}}+\ldots+k_{n} e_{i_{n}}$ e, por outro lado, existe $d \in \mathbb{K}$, não nulo, tal que $d m=0$. Logo,

$$
0=d m=\left(d k_{1}\right) e_{i_{1}}+\ldots+\left(d k_{n}\right) e_{i_{n}} .
$$

Portanto, $d k_{1}=\ldots=d k_{n}=0$ e, uma vez que $d \neq 0$ e $\mathbb{K}$ é um domínio, então $k_{1}=\ldots=$ $k_{n}=0$, isto é, $m=0$.

A proposição acima afirma que todo $\mathbb{K}$-módulo livre, em que $\mathbb{K}$ é um domínio de integridade, é livre de torção. No entanto, a recíproca não é verdadeira. Por exemplo, o $\mathbb{Z}$-módulo $\mathbb{Q}$ é livre de torção (pois se $n \in \mathbb{Z}$ é não nulo e $\frac{p}{q} \in \mathbb{Q}$, então $n \frac{p}{q}=0 \Longleftrightarrow n p=$ 
$0 \Longrightarrow p=0$ ), mas não é um $\mathbb{Z}$-módulo livre. De fato, sejam $\frac{p_{1}}{q_{1}}$ e $\frac{p_{2}}{q_{2}}$ dois racionais não nulos e, portanto $p_{1} p_{2} \neq 0$. Uma vez que

$$
\left(p_{2} q_{1}\right) \frac{p_{1}}{q_{1}}-\left(p_{1} q_{2}\right) \frac{p_{2}}{q_{2}}=0
$$

então qualquer conjunto com pelo menos dois racionais é linearmente dependente. Por outro lado, nenhum racional gera todos os racionais.

Definição 2.28. Seja $M$ um $\mathbb{K}$-módulo e $N$ um $\mathbb{K}$-submódulo de $M$, definimos o isolador de $N$ como

$$
I(N)=\{m \in M \mid \exists k \in \mathbb{K}, k \neq 0, \text { tal que } k m \in N\} .
$$

Claramente, $N \subset I(N)$. Além disso, se $\mathbb{K}$ é um domínio de integridade, então $I(N)$ é um $\mathbb{K}$-submódulo de $M$.

Proposição 2.29. Sejam $M$ um $\mathbb{K}$-módulo, em que $\mathbb{K}$ é um domínio de integridade, e $N$ um $\mathbb{K}$-submódulo de $M$. Então Tor $(M / N) \neq\{0\}$ se, e somente se, $N \varsubsetneqq I(N)$.

Demonstração. Temos que

$$
\begin{aligned}
\operatorname{Tor}(M / N) & \neq\{0\} \Longleftrightarrow \exists m \notin N \text { tal que } k m \in N \text { para algum } 0 \neq k \in \mathbb{K} \\
& \Longleftrightarrow \exists m \notin N \text { tal que } m \in I(N) \Longleftrightarrow N \varsubsetneqq I(N) .
\end{aligned}
$$

\section{$2.2 \quad$ Álgebras}

No decorrer desta seção, considere que $\mathbb{K}$ é um anel associativo, comutativo e unitário.

Definição 2.30. Uma álgebra $A$ sobre um anel $\mathbb{K}$ ou uma $\mathbb{K}$-álgebra é um módulo sobre $\mathbb{K}$ munido de uma multiplicação $\cdot: A \times A \longrightarrow A$ com as seguintes propriedades:

1. A terna $(A,+, \cdot)$ é um anel, isto é, valem as leis distributivas:

$$
\begin{gathered}
(x+y) \cdot z=x \cdot z+y \cdot z, \quad \text { para todos } x, y, z \in A \quad e \\
z \cdot(x+y)=z \cdot x+z \cdot y, \text { para todos } x, y, z \in A .
\end{gathered}
$$


2. Para todos $x, y \in A$ e $k \in \mathbb{K}$, temos

$$
k(x \cdot y)=(k x) \cdot y=x \cdot(k y) .
$$

Exemplo 2.31. Todo anel com identidade é uma $\mathbb{Z}$-álgebra. De fato, seja $(A,+, \cdot)$ um anel (com identidade). Como vimos, $(A,+)$ é um $\mathbb{Z}$-módulo. Vamos verificar que para todo inteiro $n$ e todos $x, y \in A$ é válida a igualdade $n(x \cdot y)=(n x) \cdot y$. Considere primeiro $n \geq 1$. Se $n=1$ não há nada a fazer. Suponha que a igualdade se verifique para todo inteiro $m<n$. Assim,

$$
n(x \cdot y)=(n-1)(x \cdot y)+x \cdot y=((n-1) x) \cdot y+x \cdot y=((n-1) x+x) \cdot y=(n x) \cdot y
$$

Se $n<0$, então

$$
n(x \cdot y)=|n|(-(x \cdot y))=|n|((-x) \cdot y)=(|n|(-x)) \cdot y=(n x) \cdot y \text {. }
$$

O caso em que $n=0$ é trivial.

De forma análoga verifica-se a igualdade $n(x \cdot y)=x \cdot(n y)$. Logo, A é uma $\mathbb{Z}$-álgebra.

Definição 2.32. Seja A uma $\mathbb{K}$-álgebra.

i) A é associativa se o anel $(A,+, \cdot)$ é associativo;

ii) A é comutativa se o anel $(A,+, \cdot)$ é comutativo;

iii) A é de Lie se o anel $(A,+, \cdot)$ é de Lie, isto é, dados $a, b, c \in A$, temos

$$
\begin{gathered}
a \cdot a=0 \quad(\text { a lei anticomutativa }) \\
(a \cdot b) \cdot c+(b \cdot c) \cdot a+(c \cdot a) \cdot b=0 \quad(\text { a identidade de Jacobi); }
\end{gathered}
$$

iv) A é unitária se o anel $(A,+, \cdot)$ é unitário.

Em uma álgebra associativa é possível definir uma nova operação de modo a torná-la uma álgebra de Lie. Mais precisamente, dada uma $\mathbb{K}$-álgebra associativa $A$, defina em $A$ a seguinte operação:

$$
\begin{array}{rlc}
{[,]: A \times A} & \longrightarrow & A \\
(a, b) & \longmapsto[a, b]=a b-b a
\end{array}
$$


É direto verificar que $A$ com esta nova operação é uma $\mathbb{K}$-álgebra de Lie. Essa nova álgebra, isto é, $(A,+,[]$,$) é chamada de álgebra de Lie associada à A$ e é denotada por $A^{(-)}$. Dizemos que $[a, b]$ é o comutador de $a$ e $b$. Dados $a_{1}, \ldots, a_{n} \in A, n \geq 3$, definimos indutivamente

$$
\left[a_{1}, \ldots, a_{n}\right]=\left[\left[a_{1}, \ldots, a_{n-1}\right], a_{n}\right]
$$

e denominamos comutadores normados a esquerda de comprimento $n$.

Definição 2.33. Seja A uma $\mathbb{K}$-álgebra.

1. Um submódulo $B$ de $A$ é uma subálgebra de $A$ se ele é fechado com respeito a multiplicação, isto é, $b_{1}, b_{2} \in B$ implica $b_{1} b_{2} \in B$.

2. Um submódulo I de A é um ideal bilateral (ou simplesmente ideal) de $A$ se $a_{1} b \in I$ e $b a_{2} \in I$ para todos $a_{1}, a_{2} \in A$ e $b \in I$.

Claramente todo ideal é um submódulo, no entanto a recíproca não é verdadeira.

Seja $\left\{I_{\lambda} \mid \lambda \in \Gamma\right\}$ uma família de ideais de uma $\mathbb{K}$-álgebra $A$. Pode-se mostrar que $\bigcap_{\lambda \in \Gamma} I_{\lambda}$ é um ideal de $A$. Com isso, dado $S \subset A$, o ideal gerado por $S$, denotado por $I(S)$, será a intersecção de todos os ideais de $A$ que contêm $S$. Claramente, este é o menor ideal de $A$ que contém $S$. Além disso, temos que $I(S)$ é o $\mathbb{K}$-módulo gerado pelos elementos $s, a_{1} s, s a_{2}$ e $a_{1} s a_{2}$ em que $a_{1}, a_{2} \in A$ e $s \in S$.

O centro de uma $\mathbb{K}$-álgebra $A$, denotado por $Z(A)$, é definido por

$$
Z(A):=\{a \in A \mid a b=b a \forall b \in A\}
$$

Note que o centro de uma álgebra é um exemplo de uma subálgebra que não é, necessariamente, um ideal.

Definição 2.34. Sejam $A$ e $B \quad \mathbb{K}$-álgebras. Uma aplicação $\phi: A \longrightarrow B$ é dita um homomorfismo de $\mathbb{K}$-álgebras se

(i) фé um homomorfismo de $\mathbb{K}$-módulos.

(ii) $\phi(x \cdot y)=\phi(x) \cdot \phi(y)$ para todos $x, y \in A$. 


\section{3 Álgebra livre}

No decorrer desta seção, $\mathbb{K}$ denotará um anel associativo, comutativo e unitário.

Seja $X=\left\{x_{1}, x_{2}, \ldots\right\}$ um conjunto infinito e enumerável em que os seus elementos são denominados variáveis. Definimos uma palavra em $X$ como uma sequência finita $x_{i_{1}} x_{i_{2}} \ldots x_{i_{n}}$, em que $n \in \mathbb{N}$ e $x_{i_{j}} \in X$. Definimos o tamanho (ou grau) da palavra $x_{i_{1}} x_{i_{2}} \ldots x_{i_{n}}$ como sendo $n$. Quando $n=0$, a palavra é denominada palavra vazia e é denotada por 1 . Seja $\mathcal{P}$ o conjunto de todas as palavras em $X$.

Denote por $\mathbb{K}\langle X\rangle$ o $\mathbb{K}$-módulo livre com base dada pelo conjunto $\mathcal{P}$. Os elementos de $\mathbb{K}\langle X\rangle$ são denominados polinômios. O polinômio $k_{i} x_{i_{1}} \ldots x_{i_{n}}, k_{i} \in \mathbb{K}$ e $x_{i_{j}} \in X$, também é chamado de monômio. O grau do monômio $k_{i} x_{i_{1}} \ldots x_{i_{n}}$ quando $k_{i} \neq 0$ é o grau da palavra $x_{i_{1}} \ldots x_{i_{n}}$. Note que se $f \in \mathbb{K}\langle X\rangle$, então $f=\sum_{i} m_{i}$, em que $m_{i}$ é um monômio. Se as variáveis que aparecem nos monômios de $f \in \mathbb{K}\langle X\rangle$ pertencem ao conjunto $\left\{x_{1}, x_{2}, \ldots, x_{n}\right\}$, escrevemos $f=f\left(x_{1}, x_{2}, \ldots, x_{n}\right)$.

Considere em $\mathbb{K}\langle X\rangle$ a multiplicação induzida pela seguinte multiplicação definida nos elementos de $\mathcal{P}$ :

$$
\left(x_{i_{1}} \ldots x_{i_{m}}\right) \cdot\left(x_{j_{1}} \ldots x_{j_{n}}\right)=x_{i_{1}} \ldots x_{i_{m}} x_{j_{1}} \ldots x_{j_{n}} ; x_{i_{k}}, x_{j_{l}} \in X
$$

O $\mathbb{K}$-módulo $\mathbb{K}\langle X\rangle$ munido desse produto é uma $\mathbb{K}$-álgebra associativa com unidade, que é a palavra vazia.

A $\mathbb{K}$-álgebra $\mathbb{K}\langle X\rangle$ tem a seguinte propriedade:

Proposição 2.35 (Propriedade Universal). Seja $\mathbb{K}$ um anel associativo, comutativo e unitário e A uma $\mathbb{K}$-álgebra associativa e unitária. Então qualquer aplicação $\phi: X \longrightarrow A$ pode ser estendida a um único homomorfismo $\Phi: \mathbb{K}\langle X\rangle \longrightarrow A$. Em outras palavras, existe um único homomorfismo $\Phi: \mathbb{K}\langle X\rangle \longrightarrow$ A tal que $\Phi(x)=\phi(x)$ para todo $x \in X$.

Demonstração. Defina a aplicação $\Phi: \mathbb{K}\langle X\rangle \longrightarrow A$ de forma que:

$$
\text { se } p=x_{i_{1}} \ldots x_{i_{n}} \in \mathcal{P} \text {, então } \Phi(p)=\phi\left(x_{i_{1}}\right) \ldots \phi\left(x_{i_{n}}\right) \text {. }
$$

Seja $f \in \mathbb{K}\langle X\rangle$. Então $f=\sum_{i=1}^{r} \alpha_{i} p_{i}$ em que $\alpha_{i} \in \mathbb{K}$ e $p_{i} \in \mathcal{P}$ para todo $i=1, \ldots, r$. Defina $\Phi(f)=\sum_{i=1}^{r} \alpha_{i} \phi\left(p_{i}\right)$. Pela definição de $\Phi$ temos que $\Phi(x)=\phi(x)$ para todo $x \in X$. É imediato verificar que $\Phi$ é um homomorfismo e que é o único que estende $\phi$. 
Por essa propriedade, dizemos que $\mathbb{K}\langle X\rangle$ é a álgebra associativa unitária livre, livremente gerada por $X$.

Definição 2.36. Seja $f=f\left(x_{1}, \ldots, x_{n}\right) \in \mathbb{K}\langle X\rangle$.

1) Se em cada monômio de $f$ a variável $x_{i}, i \in\{1,2, \ldots, n\}$, aparece exatamente $m$ vezes, dizemos que $f$ é homogêneo de grau $m$ na variável $x_{i}$.

2) Se $f$ for homogêneo de grau $m_{i}$ na variável $x_{i}$ para todo $i=1,2, \ldots, n$, dizemos que $f$ é multi-homogêneo de multigrau $\left(m_{1}, m_{2}, \ldots, m_{n}\right)$.

3) Se $f$ for multi-homogêneo de multigrau $\underbrace{(1,1, \ldots, 1)}_{n}$ dizemos que $f$ é multilinear de grau $n$.

Dados $m_{1}, \ldots, m_{n}$ inteiros positivos, denote por $H_{m_{1}, \ldots, m_{n}}$ o conjunto dos polinômios multi-homogêneos de multigrau $\left(m_{1}, \ldots, m_{n}\right)$ nas variáveis $x_{1}, \ldots, x_{n}$. Temos que $H_{m_{1}, \ldots, m_{n}}$ é um $\mathbb{K}$-submódulo de $\mathbb{K}\langle X\rangle$.

Denote por $P_{n}\left(x_{i_{1}}, \ldots, x_{i_{n}}\right), n \geq 1$ e $i_{1}<\ldots<i_{n}$, o conjunto dos polinômios multilineares de grau $n$ nas variáveis $x_{i_{1}}, \ldots, x_{i_{n}}$. É fácil ver que $P_{n}\left(x_{i_{1}}, \ldots, x_{i_{n}}\right)$ é um $\mathbb{K}$ módulo livre e que $\left\{x_{i_{\sigma(1)}} \ldots x_{i_{\sigma(n)}} \mid \sigma \in S_{n}\right\}$ é uma base para $P_{n}\left(x_{i_{1}}, \ldots, x_{i_{n}}\right)$. A notação simplificada $P_{n}$ será usada para $P_{n}\left(x_{1}, \ldots, x_{n}\right)$.

Sejam $f, g, h \in \mathbb{K}\langle X\rangle$. Uma vez que

$$
[f,[g, h]]=-[g, h, f]=[h, f, g]+[f, g, h]=-[f, h, g]+[f, g, h],
$$

temos a identidade

$$
[f,[g, h]]=[f, g, h]-[f, h, g]
$$

que será usada na demonstração da proposição abaixo.

Proposição 2.37. Seja $f \in \mathbb{K}\langle X\rangle$ e seja $m$ um inteiro maior que 3. Então

$$
\left[f,\left[x_{1}, x_{2}, \ldots, x_{m}\right]\right]=\sum_{\sigma \in S_{m}} k_{\sigma}\left[f, x_{\sigma(1)}, \ldots, x_{\sigma(m)}\right]
$$

em que $k_{\sigma} \in\{-1,0,1\}$. 
Demonstração. Se $m=2$, então $\left[f,\left[x_{1}, x_{2}\right]\right]=\left[f, x_{1}, x_{2}\right]-\left[f, x_{2}, x_{1}\right]$. Suponha que o resultado seja válido para algum $m \geq 2$. Temos que

$$
\begin{aligned}
& {\left[f,\left[x_{1}, x_{2}, \ldots, x_{m}, x_{m+1}\right]\right]=\left[f,\left[\left[x_{1}, x_{2}, \ldots, x_{m}\right], x_{m+1}\right]\right] } \\
= & {\left[\left[f,\left[x_{1}, x_{2}, \ldots, x_{m}\right]\right], x_{m+1}\right]-\left[\left[f, x_{m+1}\right],\left[x_{1}, x_{2}, \ldots, x_{m}\right]\right] . }
\end{aligned}
$$

Segue da hipótese de indução que

$$
\left[f,\left[x_{1}, x_{2}, \ldots, x_{m}\right]\right]=\sum_{\sigma \in S_{m}} r_{\sigma}\left[f, x_{\sigma(1)}, x_{\sigma(2)}, \ldots, x_{\sigma(m)}\right]
$$

$\mathrm{e}$

$$
\left[\left[f, x_{m+1}\right],\left[x_{1}, x_{2}, \ldots, x_{m}\right]\right]=\sum_{\sigma \in S_{m}} s_{\sigma}\left[\left[f, x_{m+1}\right], x_{\sigma(1)}, x_{\sigma(2)}, \ldots, x_{\sigma(m)}\right]
$$

em que $r_{\sigma}, s_{\sigma} \in\{-1,0,1\}$. Portanto,

$$
\begin{gathered}
{\left[f,\left[x_{1}, x_{2}, \ldots, x_{m}, x_{m+1}\right]\right]} \\
=\sum_{\sigma \in S_{m}}\left(r_{\sigma}\left[f, x_{\sigma(1)}, x_{\sigma(2)}, \ldots, x_{\sigma(m)}, x_{m+1}\right]-s_{\sigma}\left[f, x_{m+1}, x_{\sigma(1)}, x_{\sigma(2)}, \ldots, x_{\sigma(m)}\right]\right) \\
=\sum_{\delta \in S_{m+1}} k_{\delta}\left[f, x_{\delta(1)}, \ldots, x_{\delta(m+1)}\right]
\end{gathered}
$$

em que $k_{\delta} \in\{-1,0,1\}$.

Seja $\operatorname{Com}(X)$ o conjunto de todos os comutadores (normados a esquerda) de comprimento $n \geq 2$ em $X$, isto é,

$$
\operatorname{Com}(X)=\left\{\left[x_{i_{1}}, \ldots, x_{i_{t}}\right] \mid t \geq 2, x_{i_{j}} \in X\right\}
$$

Denote por $L(X)$ o $\mathbb{K}$-submódulo de $\mathbb{K}\langle X\rangle$ gerado, como módulo, por $X \cup \operatorname{Com}(X)$.

Proposição 2.38. $[f, g] \in L(X)$ para todos $f, g \in L(X)$.

Demonstração. Sejam $f, g \in X \cup \operatorname{Com}(X)$. Se $f$ e $g$ estão em $X$, então $[f, g] \in$ Com $(X) \subset L(X)$. Considere o caso em que pelo menos um dos dois polinômios não está em $X$. Sem perda de generalidade, suponha que $g \in \operatorname{Com}(X)$. Segue da proposição 2.37 que $[f, g] \in L(X)$. 
Desta forma, $L(X)$ é uma $\mathbb{K}$-subálgebra de lie de $\mathbb{K}\langle X\rangle^{(-)}$gerada, como álgebra, por $X$.

Proposição 2.39. Seja $P L_{n}=P_{n} \cap L(X)$. Então, para $n>1$, o conjunto

$$
\left\{\left[x_{n}, x_{\sigma(1)}, \ldots, x_{\sigma(n-1)}\right] \mid \sigma \in S_{n-1}\right\}
$$

é uma base do $\mathbb{K}$-módulo $P L_{n}$.

Demonstração. Denote o conjunto $\left\{\left[x_{n}, x_{\sigma(1)}, \ldots, x_{\sigma(n-1)}\right] \mid \sigma \in S_{n-1}\right\}$ por $\mathscr{L}$. Temos que um elemento de $P L_{n}$ é uma combinação linear de elementos da forma

$$
\left[x_{i_{1}}, \ldots, x_{i_{k}}, x_{n}, x_{i_{k+1}}, \ldots, x_{i_{n-1}}\right]
$$

em que $k \geq 0$ e $\left\{i_{1}, \ldots, i_{n-1}\right\}=\{1, \ldots, n-1\}$. Claramente esses elementos estão no $\mathbb{K}$-módulo gerado por $\mathscr{L}$ quando $k=0$ ou $k=1$. No caso em que $k \geq 2$, segue da identidade de Jacobi e da anticomutatividade que

$$
\begin{aligned}
& {\left[x_{i_{1}}, \ldots, x_{i_{k}}, x_{n}, x_{i_{k+1}}, \ldots, x_{i_{n-1}}\right]=\left[\left[\left[x_{i_{1}}, \ldots, x_{i_{k-1}}\right], x_{i_{k}}, x_{n}\right], x_{i_{k+1}}, \ldots, x_{i_{n-1}}\right] } \\
= & {\left[x_{n}, x_{i_{k}},\left[x_{i_{1}}, \ldots, x_{i_{k-1}}\right], x_{i_{k+1}}, \ldots, x_{i_{n-1}}\right]-\left[x_{n},\left[x_{i_{1}}, \ldots, x_{i_{k-1}}\right], x_{i_{k}}, x_{i_{k+1}}, \ldots, x_{i_{n-1}}\right] . }
\end{aligned}
$$

Assim, pelo lema $2.37,\left[x_{i_{1}}, \ldots, x_{i_{k}}, x_{n}, x_{i_{k+1}}, \ldots, x_{i_{n-1}}\right]$ está no $\mathbb{K}$-módulo gerado por $\mathscr{L}$. Seja

$$
\sum_{\sigma \in S_{n-1}} k_{\sigma}\left[x_{n}, x_{\sigma(1)}, \ldots, x_{\sigma(n-1)}\right]=0
$$

em que $k_{\sigma} \in \mathbb{K}$. Podemos escrever a equação 2.1 como

$$
\sum_{\sigma \in S_{n-1}} k_{\sigma} x_{n} x_{\sigma(1)} \ldots x_{\sigma(n-1)}+\sum_{\substack{\tau \in S_{n} \\ \tau(1) \neq n}} r_{\tau} x_{\tau(1)} \ldots x_{\tau(n)}=0
$$

Logo, $k_{\sigma}=0$ para todo $\sigma \in S_{n-1}$.

Corolário 2.40. $P L_{n}$ tem uma base formada por elementos da forma

$$
\left[x_{1}, x_{i_{2}}, \ldots, x_{i_{n}}\right]
$$

em que $\left\{i_{2}, \ldots, i_{n}\right\}=\{2, \ldots, n\}$. 
Demonstração. O argumento é análogo ao da proposição2.39.

De um modo geral, denote por $P L_{n}\left(x_{i_{1}}, \ldots, x_{i_{n}}\right), n \geq 1$ e $i_{1}<\ldots<i_{n}$, o conjunto $P_{n}\left(x_{i_{1}}, \ldots, x_{i_{n}}\right) \cap L(X)$. Claramente $P L_{n}\left(x_{i_{1}}, \ldots, x_{i_{n}}\right)$ é um $\mathbb{K}$-módulo livre que tem uma base formada por todos os comutadores multilineares nas variáveis $x_{i_{1}}, \ldots, x_{i_{n}}$ tais que $x_{i_{1}}$ é o primeiro elemento.

Denote por $B(X)$ a subálgebra de $\mathbb{K}\langle X\rangle$ gerada, como álgebra, por $\operatorname{Com}(X)$ e pela unidade de $\mathbb{K}\langle X\rangle$. Os polinômios de $B(X)$ são denominados polinômios próprios. Note que se $f \in B(X) \backslash \mathbb{K}$, então

$$
f=\sum_{i} k_{i}\left[x_{i_{(11)}}, \ldots, x_{i_{\left(1 t_{1}\right)}}\right]\left[x_{i_{(21)}}, \ldots, x_{i_{\left(2 t_{2}\right)}}\right] \ldots\left[x_{i_{(n 1)}}, \ldots, x_{i_{\left(n t_{n}\right)}}\right]
$$

em que $k_{i} \in \mathbb{K}$.

Denote por $B_{m_{1}, \ldots, m_{n}}$ o conjunto dos polinômios próprios multi-homogêneos de multigrau $\left(m_{1}, \ldots, m_{n}\right)$ nas variáveis $x_{1}, \ldots, x_{n}$, respectivamente. Desta forma $B_{m_{1}, \ldots, m_{n}}:=$ $H_{m_{1}, \ldots, m_{n}} \cap B$. Por exemplo, $\left[x_{1}, x_{2}, x_{1}, x_{2}, x_{3}\right],\left[x_{1}, x_{2}, x_{3}\right]\left[x_{1}, x_{2}\right] \in B_{2,2,1}$.

Denote por $\Gamma_{n}, n \geq 2$, o conjunto dos polinômios próprios que são multilineares de grau $n$ nas variáveis $x_{1}, \ldots, x_{n}$, isto é, $\Gamma_{n}:=B(X) \cap P_{n}$. Por exemplo,

$$
\left[x_{1}, x_{2}, x_{3}, x_{4}, x_{5}\right],\left[x_{1}, x_{3}\right]\left[x_{4}, x_{5}, x_{2}\right] \in \Gamma_{5} .
$$

Temos que $\Gamma_{n}$ é um $\mathbb{K}$-módulo.

\subsection{A base de Specht para $P_{n}$ em $\mathbb{Z}\langle X\rangle$}

Considere nesta seção que $\mathbb{K}$ é um anel associativo, comutativo e unitário. Conforme vimos anteriormente, se em uma $\mathbb{K}$-álgebra associativa e unitária $A$ definimos a operação [,] tal que $[a, b]=a b-b a$ para todos $a, b \in A$, então o $\mathbb{K}$-módulo $A$ com essa operação é uma álgebra de Lie, denominada álgebra de Lie associada à $A$ e é denotada por $A^{(-)}$. Por outro lado, dado uma $\mathbb{K}$-álgebra de Lie $G$, existe uma $\mathbb{K}$-álgebra associativa e unitária $A$ tal que $G$ possa ser mergulhada em $A^{(-)}$? A resposta a essa questão, como veremos abaixo, é dada pelo teorema de Poincaré-Birkhoff-Witt. Usamos esse teorema para exibir uma base para $P_{n}$ em $\mathbb{Z}\langle X\rangle$, denominada base de Specht. 
Definição 2.41. Seja $G$ uma $\mathbb{K}$-álgebra de Lie. Uma álgebra envelopante universal de $G$ é um par $(U(G), \epsilon)$ em que

i) $U(G)$ é uma $\mathbb{K}$-álgebra associativa unitária;

ii) $\epsilon: G \longrightarrow U(G)^{(-)}$é um homomorfismo de álgebras de Lie;

ii) satisfaz a seguinte propriedade universal: para toda $\mathbb{K}$-álgebra associativa unitária $A$ e todo homomorfismo de $\mathbb{K}$-álgebras de Lie $\phi: G \longrightarrow A^{(-)}$, existe um único homomorfismo de $\mathbb{K}$-álgebras associativas unitárias $\psi: U(G) \longrightarrow$ A tal que $\psi \epsilon=\phi$.

Proposição 2.42. Para toda $\mathbb{K}$-álgebra de Lie $G$, a álgebra envelopante universal de $G$ existe e é única a menos de isomorfismos.

Demonstração. Veja [5] páginas 57 e 58.

Exemplo 2.43. Lembramos que $L(X)$ foi definido como sendo o $\mathbb{K}$-submódulo de $\mathbb{K}\langle X\rangle$ gerado por $X \cup \operatorname{Com}(X)$ em que $\operatorname{Com}(X)=\left\{\left[x_{i_{1}}, \ldots, x_{i_{t}}\right] \mid t \geq 2, x_{i_{j}} \in X\right\}$. Além disso, conforme vimos na seção anterior, $L(X)$ é uma subálgebra de Lie de $\mathbb{K}\langle X\rangle^{(-)}$. Considere então a inclusão $\iota: L(X) \hookrightarrow \mathbb{K}\langle X\rangle^{(-)}$e sejam $A$ uma $\mathbb{K}$-álgebra associativa unitária e $\phi: L(X) \longrightarrow A^{(-)}$um homomorfismo de álgebras de Lie. A aplicação $\phi_{0}: X \longrightarrow A$ definida por $\phi_{0}(x)=\phi(x), x \in X$, induz um único homomorfismo $\psi: \mathbb{K}\langle X\rangle \longrightarrow A$ tal que $\psi(x)=\phi(x)$. Assim, $\psi \iota=\phi e$, portanto, $(\mathbb{K}\langle X\rangle, \iota)$ é a álgebra envelopante universal de $L(X)$.

Proposição 2.44 (O Teorema de Poincaré-Birkhoff-Witt).

Seja $G$ uma $\mathbb{K}$-álgebra de Lie e $(U(G), \epsilon)$ sua álgebra envelopante universal. Se $G$ é um $\mathbb{K}$-módulo livre e $E=\left\{e_{i} \mid i \in I\right\}$ é uma base bem ordenada para $G$, então $U(G)$ é um $\mathbb{K}$-módulo livre com base dada pelos elementos da forma

$$
\epsilon\left(e_{i_{1}}\right) \cdots \epsilon\left(e_{i_{p}}\right), i_{1} \leq \cdots \leq i_{p}, i_{k} \in I, p=0,1,2, \ldots
$$

Em particular, $\epsilon: G \longrightarrow U(G)^{(-)}$é injetivo.

Demonstração. Veja [5] páginas 58 e 59. 
Seja $G$ uma $\mathbb{K}$-álgebra de Lie gerada por um conjunto $X=\left\{x_{1}, x_{2}, \ldots\right\}$ e seja $F$ uma $\mathbb{K}$-álgebra de Lie arbitrária. Se para toda aplicação $\phi_{0}: X \longrightarrow F$, existir um único homomorfismo $\phi: G \longrightarrow F$ tal que $\phi(x)=\phi_{0}(x)$ para todo $x \in X$, então $G$ é denominada álgebra de Lie livre, livremente gerada pelo conjunto $X$.

Proposição 2.45. Se $G$ é a $\mathbb{K}$-álgebra de Lie livre, livremente gerada pelo conjunto $X$, então $L(X) \cong G$.

Demonstração. Considere a aplicação $\varphi_{0}: X \longrightarrow X \subset L(X)$ definida por $\varphi_{0}(x)=x$ para todo $x \in X$. Uma vez que $G$ é a álgebra de Lie livre, livremente gerada por $X$, existe um único homomorfismo de álgebras de Lie $\varphi: G \longrightarrow L(X)$ tal que $\varphi(x)=\varphi_{0}(x)=x$.

Seja $U$ a álgebra envelopante de $G$. É conhecido que $G$ é um $\mathbb{K}$-módulo livre (veja, por exemplo [5] páginas 48 e 49). Desta forma, pelo teorema de Poincare-Birkhoff-Witt, $G$ é isomorfo a uma subálgebra de Lie de $U^{(-)}$. Sem perda de generalidade, suponha que $G \subset U$. Considere a aplicação $\psi_{0}: X \longrightarrow X \subset G$ definida por $\psi_{0}(x)=x$. Uma vez que $\mathbb{K}\langle X\rangle$ é a álgebra associativa unitária livre, livremente gerada por $X$, existe um único homomorfismo de álgebras associativas unitárias $\psi: \mathbb{K}\langle X\rangle \longrightarrow U$ tal que $\psi(x)=\psi_{0}(x)=x$. Note que $\psi(L(X)) \subset G$. Portanto, se $\widetilde{\psi}$ é a restrição de $\psi$ a $L(X)$, então $\widetilde{\psi}$ é um homomorfismo (de álgebras de Lie) de $L(X)$ em $G$ tal que $\widetilde{\psi}(x)=x$.

Vimos que uma base linear para $P_{n}$, o $\mathbb{K}$-submódulo de $\mathbb{K}\langle X\rangle$ dos polinômios multilineares nas variáveis $x_{1}, \ldots, x_{n}$, é dada pelo conjunto $\left\{x_{\sigma(1)} \ldots x_{\sigma(n)} \mid \sigma \in S_{n}\right\}$. Se $\mathbb{K}=\mathbb{Z}$, então existe uma base ordenada para $P_{n}$ conhecida como base de Specht. Na sequência desta seção será feita a construção dessa base. Para tanto, $P_{n}$ e $L(X)$ serão vistos como $\mathbb{Z}$-submódulos de $\mathbb{Z}\langle X\rangle$. Vale lembrar que qualquer $\mathbb{Z}$-submódulo de $\mathbb{Z}\langle X\rangle$ é livre (proposição 2.22).

Definição 2.46. Seja $n \geq 1$.

(1) Seja $\left\{i_{1}, \ldots, i_{k}\right\}$ um subconjunto de $\{1, \ldots, n\}$. O monômio $x_{i_{1}} \ldots x_{i_{k}}$ é denominado regular se $i_{1}<\ldots<i_{k}$. O comutador multilinear $\left[x_{i_{1}}, x_{i_{2}}, \ldots, x_{i_{k}}\right]$ é denominado regular se $i_{1}$ é o menor elemento do conjunto $\left\{i_{1}, \ldots, i_{k}\right\}$. 
(2) O produto multilinear $C W \in P_{n}$, em que $C=C_{1} \ldots C_{s}(s \geq 0)$ é um produto de comutadores regulares e $W$ é um monômio regular ou é 1 , é também denominado regular se

(a) o grau de $C_{i}$ não cresce da esquerda para direita; $e$

(b) o indice da indeterminada inicial de comutadores $C_{i}$ de mesmo grau cresce $d a$ esquerda para direita.

Exemplo 2.47. O conjunto dos produtos regulares de $P_{1}, P_{2}$ e $P_{3}$ são, respectivamente, iguais a

$\left\{x_{1}\right\}, \quad\left\{x_{1} x_{2},\left[x_{1}, x_{2}\right]\right\}$ e $\left\{x_{1} x_{2} x_{3},\left[x_{1}, x_{2}\right] x_{3},\left[x_{1}, x_{3}\right] x_{2},\left[x_{2}, x_{3}\right] x_{1},\left[x_{1}, x_{2}, x_{3}\right],\left[x_{1}, x_{3}, x_{2}\right]\right\}$.

Proposição 2.48. O conjunto dos produtos regulares de $P_{n}(n \geq 1)$ gera $P_{n}$ como $\mathbb{Z}$ módulo.

Demonstração. Temos que $P_{1}$ é gerado por $x_{1}$, que é um produto regular de $P_{1}$. Seja $n$ um inteiro maior que 1 e suponha que para todo $m<n, P_{m}$ seja gerado pelos seus produtos regulares. Para verificar que o conjunto dos produtos regulares de $P_{n}(n \geq 1)$ geram $P_{n}$ é suficiente mostrar que os monômios $q_{\sigma}=x_{\sigma(1)} x_{\sigma(2)} \ldots x_{\sigma(n)}$ em que $\sigma \in S_{n}$ são combinações lineares de produtos regulares de $P_{n}$. Se $\sigma=1$ (identidade), então $q_{\sigma}$ é um produto regular. Para o caso em que $\sigma \neq 1$, usando as relações $a b=b a+[a, b]$ e $a[b, c]=[b, c] a-[b, c, a]$ em que $a, b, c \in \mathbb{Z}\langle X\rangle$, podemos escrever $q_{\sigma}$ como uma combinação linear de $x_{1} x_{2} \ldots x_{n}$ com elementos da forma

$$
\left[x_{j_{1}}, \ldots, x_{j_{r}}\right] x_{k_{1}} \ldots x_{k_{n-r}} \text { em que } r \geq 2 \text { e }\left\{j_{1}, \ldots, j_{r}, k_{1}, \ldots, k_{n-r}\right\}=\{1, \ldots, n\} \text {. }
$$

De acordo com o corolário 2.40, podemos supor que $j_{1}$ é o elemento minimal do conjunto $\left\{j_{1}, \ldots, j_{r}\right\}$. Pela hipótese de indução, o monômio $x_{k_{1}} \ldots x_{k_{n-r}}$ pode ser escrito como uma combinação de produtos regulares de $P_{n-r}\left(x_{k_{1}}, \ldots, x_{k_{n-r}}\right)$. Assim $\left[x_{j_{1}}, \ldots, x_{j_{r}}\right] x_{k_{1}} \ldots x_{k_{n-r}}$ pode ser escrito como uma combinação linear de elementos da forma $C_{1} C_{2} \ldots C_{s} W$ em que $C_{i}$ é um comutador regular e $W$ é um monômio regular (ou é 1). No entanto, pode ocorrer da ordem dos comutadores $C_{1}, \ldots, C_{s}$ não estar de acordo com a definição de produto regular. Nesse caso, basta usar a relação $C_{i} C_{j}=C_{j} C_{i}+\left[C_{i}, C_{j}\right]$ e o fato de que $\left[C_{i}, C_{j}\right] \in L(X)$ (proposição 2.38). 
Exemplo 2.49. $O$ monômio $x_{2} x_{4} x_{1} x_{3}$ de $P_{4}$ pode ser escrito como

$$
\begin{gathered}
x_{1} x_{2} x_{3} x_{4}-\left[x_{3}, x_{4}\right] x_{1} x_{2}-\left[x_{1}, x_{2}\right] x_{3} x_{4}-\left[x_{1}, x_{4}\right] x_{2} x_{3} \\
{\left[x_{1}, x_{4}, x_{3}\right] x_{2}-\left[x_{1}, x_{3}, x_{4}\right] x_{2}+\left[x_{2}, x_{4}, x_{3}\right] x_{1}-\left[x_{2}, x_{3}, x_{4}\right] x_{1}+\left[x_{1}, x_{4}, x_{2}\right] x_{3}} \\
+\left[x_{1}, x_{2}, x_{4}, x_{3}\right]-\left[x_{1}, x_{2}, x_{3}, x_{4}\right]-\left[x_{1}, x_{4}, x_{3}, x_{2}\right]+\left[x_{1}, x_{3}, x_{4}, x_{2}\right]+\left[x_{1}, x_{2}\right]\left[x_{3}, x_{4}\right] .
\end{gathered}
$$

Seja $\tilde{X}$ o $\mathbb{Z}$-submódulo de $\mathbb{Z}\langle X\rangle$ gerado por $X$ e seja $C_{\left(i_{1}, \ldots, i_{k}\right)}^{\left(m_{i_{1}}, \ldots, m_{i_{k}}\right)}(k \geq 2)$ o $\mathbb{Z}$ submódulo de $\mathbb{Z}\langle X\rangle$ gerado pelos comutadores de multigrau $\left(m_{i_{1}}, \ldots, m_{i_{k}}\right)$ nas variáveis $x_{i_{1}}, \ldots, x_{i_{k}}$ em que $i_{1}<\ldots<i_{k}$. Por exemplo, $C_{(1,2)}^{(2,2)}$ é gerado pelos comutadores $\left[x_{1}, x_{2}, x_{1}, x_{2}\right],\left[x_{1}, x_{2}, x_{2}, x_{1}\right],\left[x_{2}, x_{1}, x_{1}, x_{2}\right]$ e $\left[x_{2}, x_{1}, x_{2}, x_{1}\right]$. Temos que

$$
L(X)=\tilde{X} \oplus\left(\bigoplus_{\substack{m_{j}>1 \\ \text { para algum } j}} C_{\left(i_{1}, \ldots, i_{k}\right)}^{\left(m_{i_{1}}, \ldots, m_{i_{k}}\right)}\right) \oplus\left(\bigoplus C_{\left(i_{1}, \ldots, i_{k}\right)}^{(1, \ldots, 1)}\right) .
$$

Uma vez que $C_{\left(i_{1}, \ldots, i_{k}\right)}^{\left(1, \ldots, i_{1}\right)}=P L_{k}\left(x_{i_{1}}, \ldots, x_{i_{k}}\right)$, segue do corolário 2.40 que uma base para esse $\mathbb{Z}$-módulo é dada pelos comutadores multilineares em $x_{i_{1}}, \ldots, x_{i_{k}}$ em que $x_{i_{1}}$ é primeiro elemento. Denote essa base por $\mathscr{C}_{\left(i_{1}, \ldots, i_{k}\right)}^{(1, \ldots, 1)}$. Por exemplo, $\mathscr{C}_{(2,4,6)}^{(1,1,1)}=\left\{\left[x_{2}, x_{4}, x_{6}\right],\left[x_{2}, x_{6}, x_{4}\right]\right\}$. Seja $\mathscr{C}$ uma base arbitrária para o $\mathbb{Z}$-módulo $\bigoplus_{m_{j}>1} C_{\left(i_{1}, \ldots, i_{k}\right)}^{\left(m_{i_{1}}, \ldots, m_{i_{k}}\right)}$. Assim

$$
X \cup \mathscr{C} \cup\left(\bigcup \mathscr{C}_{\left(i_{1}, \ldots, i_{k}\right)}^{(1, \ldots, 1)}\right)
$$

é uma base para $L(X)$. Ordene essa base de forma que

1) $x_{i}<x_{j}$ se $i<j$,

2) os comutadores precedam as variáveis,

3) os comutadores de maior grau estejam antes dos de menor grau, e

4) se dois comutadores multilineares tiverem o mesmo grau, aquele com a primeira variável menor estará antes do outro.

Tendo em vista que $U(L(X))=\mathbb{Z}\langle X\rangle$ e tomando para $L(X)$ a base $X \cup \mathscr{C} \cup$ $\left(\bigcup \mathscr{C}_{\left(i_{1}, \ldots, i_{k}\right)}^{(1, \ldots, 1)}\right)$ com a ordenação dada acima, obtemos (pelo teorema de Poincaré-BirkhoffWitt) uma base $\mathscr{B}$ para $\mathbb{Z}\langle X\rangle$ de tal forma que $\mathscr{B} \cap P_{n}$ é o conjunto dos produtos regulares de $P_{n}$. Segue então que o conjunto dos produtos regulares de $P_{n}$ formam uma base linear para $P_{n}$, denominada base de Specht. 


\subsection{Fatos relacionados a uma álgebra livre sobre um corpo}

Ao longo desta seção, $\mathbb{K}$ é um corpo de característica zero.

Denote por $T^{(m)}$ o ideal bilateral de $\mathbb{K}\langle X\rangle$ gerado como ideal pelos elementos

$$
\left[a_{1}, \ldots, a_{m}\right]
$$

em que $a_{1}, \ldots, a_{m} \in \mathbb{K}\langle X\rangle, m>1$. Note que $T^{(m)}$ é o $\mathbb{K}$-espaço vetorial gerado pelos elementos $b_{1}\left[a_{1}, \ldots, a_{m}\right] b_{2}$ em que $a_{1}, \ldots, a_{m}, b_{1}, b_{2} \in \mathbb{K}\langle X\rangle$.

Definição 2.50. Um ideal $I$ de $\mathbb{K}\langle X\rangle$ é dito um $T$-ideal se $\varphi(I) \subset I$ para todo endomorfismo $\varphi$ de $\mathbb{K}\langle X\rangle$.

Claramente $T^{(m)}$ é um $T$-ideal de $\mathbb{K}\langle X\rangle$.

Proposição 2.51. Sejam $\sigma \in S_{4}$ e $a_{1}, a_{2}, a_{3}, a_{4}, f, g, h \in \mathbb{K}\langle X\rangle$. Então

$$
f\left[a_{1}, a_{2}\right] g\left[a_{3}, a_{4}\right] h-(-1)^{\sigma} f\left[a_{\sigma(1)}, a_{\sigma(2)}\right] g\left[a_{\sigma(3)}, a_{\sigma(4)}\right] h \in T^{(3)} .
$$

Demonstração. Uma vez que $[b, c d]=c[b, d]+[b, c] d$ para todo $b, c, d \in \mathbb{K}\langle X\rangle$, então

$$
\left[a_{1}, a_{2} a_{3}, a_{4}\right]=\left[a_{1}, a_{2}\right]\left[a_{3}, a_{4}\right]+\left[a_{2}, a_{4}\right]\left[a_{1}, a_{3}\right]+\left[a_{1}, a_{2}, a_{4}\right] a_{3}+a_{2}\left[a_{1}, a_{3}, a_{4}\right]
$$

e, portanto, $\left[a_{1}, a_{2}\right]\left[a_{3}, a_{4}\right]+\left[a_{2}, a_{4}\right]\left[a_{1}, a_{3}\right] \in T^{(3)}$. Como

$$
\left[a_{2}, a_{4}\right]\left[a_{1}, a_{3}\right]-\left[a_{1}, a_{3}\right]\left[a_{2}, a_{4}\right]=\left[\left[a_{2}, a_{4}\right],\left[a_{1}, a_{3}\right]\right] \in T^{(3)},
$$

temos que

$$
\left[a_{1}, a_{2}\right]\left[a_{3}, a_{4}\right]+\left[a_{1}, a_{3}\right]\left[a_{2}, a_{4}\right] \in T^{(3)} .
$$

Segue disso e da anticomutatividade que

$$
\left[a_{1}, a_{2}\right]\left[a_{3}, a_{4}\right]-(-1)^{\sigma}\left[a_{\sigma(1)}, a_{\sigma(2)}\right]\left[a_{\sigma(3)}, a_{\sigma(4)}\right] \in T^{(3)} .
$$

Assim, dado $\sigma \in S_{4}$, temos

$$
f\left[a_{1}, a_{2}\right]\left[a_{3}, a_{4}\right] g h-(-1)^{\sigma} f\left[a_{\sigma(1)}, a_{\sigma(2)}\right]\left[a_{\sigma(3)}, a_{\sigma(4)}\right] g h \in T^{(3)} .
$$

Mas,

$$
f\left[a_{1}, a_{2}\right]\left[a_{3}, a_{4}\right] g h=f\left[a_{1}, a_{2}\right] g\left[a_{3}, a_{4}\right] h+f\left[a_{1}, a_{2}\right]\left[a_{3}, a_{4}, g\right] h .
$$

E, uma vez que $f\left[a_{1}, a_{2}\right]\left[a_{3}, a_{4}, g\right] h \in T^{(3)}$, segue o resultado. 
Corolário 2.52. Sejam $\sigma \in S_{2 k}, 2 k \geq 4$, e $a_{1}, a_{2}, \ldots, a_{2 k} \in \mathbb{K}\langle X\rangle$. Então

$$
\left[a_{1}, a_{2}\right]\left[a_{3}, a_{4}\right] \cdots\left[a_{2 k-1}, a_{2 k}\right]-(-1)^{\sigma}\left[a_{\sigma(1)}, a_{\sigma(2)}\right]\left[a_{\sigma(3)}, a_{\sigma(4)}\right] \cdots\left[a_{\sigma(2 k-1)}, a_{\sigma(2 k)}\right] \in T^{(3)}
$$

Observação 2.53. O corolário 2.52 é válido em $\mathbb{Z}\langle X\rangle$.

Corolário 2.54. Seja

$$
f=\left[a_{1}, a_{2}\right]\left[a_{3}, a_{4}\right] \ldots\left[a_{2 k-1}, a_{2 k}\right]
$$

em que $k \geq 1$ e $a_{i} \in \mathbb{K}\langle X\rangle$ para todo $i=1, \ldots, 2 k$. Se $a_{i}=a_{j}$ para algum par $i, j \in$ $\{1, \ldots, 2 k\}$ com $i \neq j$, então $f \in T^{(3)}$.

Demonstração. Se $a_{i}$ e $a_{j}$ não estiverem no mesmo comutador, tome uma permutação $\sigma \in S_{2 k}$ tal que $\sigma(1)=i$ e $\sigma(2)=j$. Assim, pela proposição 2.52, $f \in T^{(3)}$.

Lembramos que $H_{a_{1}, \ldots, a_{k}}$ é o conjunto dos polinômios multi-homogêneos de multigrau $\left(a_{1}, \ldots, a_{k}\right)$ nas variáveis $x_{1}, \ldots, x_{k}$ e que $B_{a_{1}, \ldots, a_{k}}=H_{a_{1}, \ldots, a_{k}} \cap B$, em que $B$ é o conjunto dos polinômios próprios. Note que, no contexto desta seção, $H_{a_{1}, \ldots, a_{k}}$ e $B_{a_{1}, \ldots, a_{k}}$ são $\mathbb{K}$ espaços vetoriais.

Proposição 2.55. Seja $T$ um $T$-ideal de $\mathbb{K}\langle X\rangle$ e considere que $\pi(A)$ é a imagem de um subconjunto $A$ de $\mathbb{K}\langle X\rangle$ pelo homomorfismo canônico $\pi: \mathbb{K}\langle X\rangle \longrightarrow \mathbb{K}\langle X\rangle / T$. Sejam $m_{1}, \ldots, m_{k}$ inteiros positivos e seja $W$ o conjunto formado por todas as sequência de inteiros $a=\left(a_{1}, \ldots, a_{k}\right)$ tais que $0 \leq a_{i} \leq m_{i}$ para todo $i=1, \ldots, k$. Suponha que para todo $a \in W$ tal que $B_{a} \not \subset T$, exista um subconjunto $Q_{a}=\left\{q_{a}^{\left(j_{a}\right)} \mid j_{a} \in J_{a}\right\}$ de $B_{a}$ tal que $\pi\left(Q_{a}\right)$ é uma base de $\pi\left(B_{a}\right)$. Então $\pi(\mathcal{Q})$, em que

$$
\mathcal{Q}=\left\{q_{a}^{\left(j_{a}\right)} x_{1}^{m_{1}-a_{1}} \ldots x_{k}^{m_{k}-a_{k}} \mid q_{a}^{\left(j_{a}\right)} \in Q_{a}, a \in W\right\}
$$

é uma base de $\pi\left(H_{m}\right)$ em que $m=\left(m_{1}, \ldots, m_{k}\right)$.

Demonstração. Seja $f \in H_{m}$. Escreva $f$ na forma

$$
f=\sum_{a} \omega_{a}\left(x_{1}, \ldots, x_{k}\right) x_{1}^{m_{1}-a_{1}} \ldots x_{k}^{m_{k}-a_{k}}
$$

em que $\omega_{a}\left(x_{1}, \ldots, x_{k}\right) \in B_{a}$ (veja [15], página 43). Assim

$$
f+T=\sum_{a} \omega_{a}\left(x_{1}, \ldots, x_{k}\right) x_{1}^{m_{1}-a_{1}} \ldots x_{k}^{m_{k}-a_{k}}+T
$$




$$
=\sum_{a} \sum_{j_{a}} \alpha_{j_{a}} q_{a}^{\left(j_{a}\right)} x_{1}^{m_{1}-a_{1}} \ldots x_{k}^{m_{k}-a_{k}}+T, \quad \alpha_{j_{a}} \in \mathbb{K} .
$$

Desta forma, $\pi(\mathcal{Q})$ gera $\pi\left(H_{m}\right)$.

Seja $g=\sum_{\left(a, j_{a}\right)} \alpha_{\left(a, j_{a}\right)} q_{a}^{\left(j_{a}\right)} x_{1}^{m_{1}-a_{1}} \ldots x_{k}^{m_{k}-a_{k}}, \alpha_{\left(a, j_{a}\right)} \in \mathbb{K}$, e suponha que $g \in T$. Escrevendo

$$
g=\sum_{a}\left(\sum_{j_{a}} \alpha_{\left(a, j_{a}\right)} q_{a}^{\left(j_{a}\right)}\right) x_{1}^{m_{1}-a_{1}} \ldots x_{k}^{m_{k}-a_{k}}
$$

então de acordo com [15] (proposição 4.3.3)

$$
\sum_{j_{a}} \alpha_{\left(a, j_{a}\right)} q_{a}^{\left(j_{a}\right)} \in T, \text { para cada } a
$$

Logo, $\alpha_{\left(a, j_{a}\right)}=0$ e, portanto, o conjunto $\pi(\mathcal{Q})$ é linearmente independente.

Por meio da proposição acima pode-se obter uma base para o $\mathbb{K}$-espaço vetorial

$$
H_{m_{1}, \ldots, m_{k}} /\left(T^{(3)} \cap H_{m_{1}, \ldots, m_{k}}\right) .
$$

Para tanto, serão feitas algumas considerações.

Sejam $a_{1}, \ldots, a_{k}$ inteiros positivos e $f \in B_{a_{1}, \ldots, a_{k}}$. Sabe-se que $f$ é uma combinação linear de produtos de comutadores em que pelo menos um dos comutadores tem comprimento maior ou igual a 3 (tipo 1) ou todos os comutadores têm comprimento igual a 2 (tipo 2). Considere os seguintes casos:

1) $\sum_{q=1}^{k} a_{q}$ é ímpar;

Então $f$ é uma combinação linear de produtos de comutadores do tipo 1. Logo, $f \in T^{(3)}$ e, portanto

$$
B_{a_{1}, \ldots, a_{k}} \subset T^{(3)}
$$

2) $\sum_{q=1}^{k} a_{q}$ é par e $a_{i} \geq 2$ para algum $i \in\{1, \ldots, k\}$.

Então $f$ é uma combinação linear de produtos de comutadores que podem ser do tipo 1 ou do tipo 2. Mas, pelo corolário 2.54, o produto de comutadores do tipo 2 com elementos repetidos está em $T^{(3)}$. Logo, $f \in T^{(3)}$ e, portanto

$$
B_{a_{1}, \ldots, a_{k}} \subset T^{(3)}
$$


3) $\sum_{q=1}^{k} a_{q}$ é par e $a_{i}=1$ para todo $i=1, \ldots, k$.

Nesse caso, $k=2 t$ para algum $t \geq 1$. Então, pelo corolário 2.52, temos que

$$
f+T^{(3)}=\alpha\left[x_{1}, x_{2}\right] \ldots\left[x_{2 t-1}, x_{2 t}\right]+T^{(3)},
$$

em que $\alpha \in \mathbb{K}$. Pode-se mostrar que $\left[x_{1}, x_{2}\right] \ldots\left[x_{2 t-1}, x_{2 t}\right] \notin T^{(3)}$ (veja, por exemplo, [15], teorema 5.1.2, página 50). Portanto, $\left\{\left[x_{1}, x_{2}\right] \ldots\left[x_{2 t-1}, x_{2 t}\right]+T^{(3)}\right\}$ é uma base para $\pi(\underbrace{B_{1, \ldots, 1}^{1, \ldots}}_{2 t})$

Segue dessas considerações e da proposição 2.55 o seguinte resultado:

Proposição 2.56. Sejam $m_{1}, \ldots, m_{k}$ inteiros não nulos e seja $\mathcal{S}$ o conjunto formado pelos elementos da forma

$$
\left[x_{i_{1}}, x_{i_{2}}\right] \cdots\left[x_{i_{2 t-1}}, x_{i_{2 t}}\right] x_{1}^{b_{1}} \ldots x_{k}^{b_{k}}
$$

em que

- $b_{j} \in\left\{m_{j}, m_{j}-1\right\}, j=1, \ldots, k$;

- $i_{1}<i_{2}<\ldots<i_{2 t}, \quad 0 \leq 2 t \leq k$, são os inteiros $j \in\{1,2, \ldots, k\}$ para os quais $b_{j}=m_{j}-1$.

Então o conjunto

$$
\left\{f+T^{(3)} \mid f \in \mathcal{S}\right\}
$$

é uma base do $\mathbb{K}$-espaço vetorial $H_{m_{1}, \ldots, m_{k}} /\left(T^{(3)} \cap H_{m_{1}, \ldots, m_{k}}\right)$.

Corolário 2.57. O $\mathbb{K}$-espaço vetorial $P_{k} /\left(T^{(3)} \cap P_{k}\right)$ tem uma base cujos elementos são

$$
\left[x_{i_{1}}, x_{i_{2}}\right] \cdots\left[x_{i_{2 t-1}}, x_{i_{2 t}}\right] x_{j_{1}} \cdots x_{j_{k-2 t}}+T^{(3)}
$$

em que $i_{1}<\cdots<i_{2 t}, j_{1}<\cdots<j_{k-2 t}$ e $0 \leq 2 t \leq k$.

Observação 2.58. Se tomarmos $\mathbb{K}=\mathbb{Q}$ temos que os elementos $f+T^{(3)}$ em que $f \in \mathcal{S}$ são linearmente independentes em $H_{m_{1}, \ldots, m_{k}} /\left(T^{(3)} \cap H_{m_{1}, \ldots, m_{k}}\right)$ visto como $\mathbb{Z}$-módulo. 


\subsection{A série central inferior de uma álgebra de Lie}

Sejam $\mathbb{K}$ um anel comutativo, associativo e unitário. Como vimos anteriormente, uma $\mathbb{K}$-álgebra $G$ é dita uma álgebra de Lie se, o seu produto é anti-simétrico e satisfaz a identidade de Jacobi. Vamos denotar a operação em $G$ por [,]. Assim

1) $[f, g]=-[g, f]$ para todos $f, g \in G$. (anti-simétrica)

2) $[[f, g], h]+[[g, h], f]+[[h, f], g]=0$. (identidade de Jacobi)

Naturalmente, as definições e resultados dados anteriormente para $\mathbb{K}$-álgebras se aplicam as álgebras de Lie, que são casos particulares. No entanto, dadas as propriedades extras da álgebra de Lie, algumas definições podem ser reformuladas. Por exemplo:

1) Um submódulo $I$ de uma $\mathbb{K}$-álgebra de lie $G$ é um ideal de $G$ se $[g, f] \in I$ para todos $g \in G$ e $f \in I$

2) O centro de uma $\mathbb{K}$-álgebra de lie $G, Z(G)$, é definido por

$$
Z(G):=\{g \in G \mid[g, f]=0, \forall f \in G\}
$$

Note que nessas condições, $Z(G)$ é um ideal de $G$.

Sejam $G$ uma $\mathbb{K}$-álgebra de lie e $A, B \subset G$. Denotamos por $[A, B]$ o $\mathbb{K}$-submódulo de $G$ gerado pelos elementos $[a, b]$ em que $a \in A$ e $b \in B$. Note que se $A$ e $B$ forem ideais de $G$, então $[A, B]$ é um ideal de $G$.

Defina a série de ideais de Lie em $G$ indutivamente:

$$
\begin{gathered}
L^{(1)}(G)=G \\
L^{(2)}(G)=\left[L^{(1)}(G), G\right]=[G, G] ; \\
L^{(3)}(G)=\left[L^{(2)}(G), G\right]=[G, G, G] ; \\
\vdots \\
L^{(i+1)}(G)=\left[L^{(i)}(G), G\right]=\underbrace{[G, G, \ldots, G]}_{i+1 \text { vezes }} .
\end{gathered}
$$


Essa série é denominada de série central inferior de $G$. Temos que

$$
G=L^{(1)}(G) \supset L^{(2)}(G) \supset L^{(3)}(G) \supset \ldots
$$

Defina os sucessivos quocientes da série central por $B_{i}(G):=L^{(i)}(G) / L^{(i+1)}(G)$. Abrevie $L^{(i)}(G)$ como $L^{(i)}$ e $B_{i}(G)$ como $B_{i}$. Para todos $i, j \geq 1$, considere as aplicações $\phi_{i j}$ : $B_{i} \times B_{j} \rightarrow B_{i+j}$ definidas por

$$
\phi_{i j}\left(a+L^{(i+1)}, b+L^{(j+1)}\right)=[a, b]+L^{(i+j+1)}, a \in L^{(i)}, \text { e } b \in L^{(j)} .
$$

Note que $\left[L^{(i)}, L^{(j)}\right] \subset L^{(i+j)}$ e, portanto, $[a, b] \in L^{(i+j)}$. Para verificar que $\phi_{i j}$ está bem definida, considere $a, a^{\prime} \in L^{(i)}$ e $b, b^{\prime} \in L^{(j)}$ tais que $a-a^{\prime} \in L^{(i+1)}$ e $b-b^{\prime} \in L^{(j+1)}$. Desta forma,

$$
\left[a, b-b^{\prime}\right] \in\left[L^{(i)}, L^{(j+1)}\right] \subset L^{(i+j+1)} \text { e }\left[a-a^{\prime}, b^{\prime}\right] \in\left[L^{(i+1)}, L^{(j)}\right] \subset L^{(i+j+1)} .
$$

Portanto, $[a, b]-\left[a^{\prime}, b^{\prime}\right] \in L^{(i+j+1)}$. Note que $\phi_{i j}$ é uma aplicação bilinear.

Proposição 2.59. Sejam $\tilde{a} \in B_{i}, \tilde{b} \in B_{j}$ e $\tilde{c} \in B_{k}$. Então

(i) $\phi_{i i}(\tilde{a}, \tilde{a})=0$;

(ii) $\phi_{i j}(\tilde{a}, \tilde{b})+\phi_{j i}(\tilde{b}, \tilde{a})=0$;

(iii) $\phi_{(i+j) k}\left(\phi_{i j}(\tilde{a}, \tilde{b}), \tilde{c}\right)+\phi_{(j+k) i}\left(\phi_{j k}(\tilde{b}, \tilde{c}), \tilde{a}\right)+\phi_{(k+i) j}\left(\phi_{k i}(\tilde{c}, \tilde{a}), \tilde{b}\right)=0$.

Demonstração. Tome $a \in L^{(i)}, b \in L^{(j)}$ e $c \in L^{(k)}$ tais que $\tilde{a}=a+L^{(i+1)}, \tilde{b}=b+L^{(j+1)} \mathrm{e}$ $\tilde{c}=c+L^{(k+1)}$. Desta forma,

i) $\phi_{i i}(\tilde{a}, \tilde{a})=[a, a]+L^{(2 i+1)}=L^{(2 i+1)}$;

ii) $\phi_{i j}(\tilde{a}, \tilde{b})+\phi_{j i}(\tilde{b}, \tilde{a})=[a, b]+[b, a]+L^{(i+j+1)}=L^{(i+j+1)}$;

iii) $\phi_{(i+j) k}\left(\phi_{i j}(\tilde{a}, \tilde{b}), \tilde{c}\right)+\phi_{(j+k) i}\left(\phi_{j k}(\tilde{b}, \tilde{c}), \tilde{a}\right)+\phi_{(k+i) j}\left(\phi_{k i}(\tilde{c}, \tilde{a}), \tilde{b}\right)=$

$$
\begin{aligned}
& \phi_{(i+j) k}\left([a, b]+L^{(i+j+1)}, \tilde{c}\right)+\phi_{(j+k) i}\left([b, c]+L^{(j+k+1)}, \tilde{a}\right)+ \\
& \phi_{(k+i) j}\left([c, a]+L^{(i+k+1)}, \tilde{b}\right)=[a, b, c]+[b, c, a]+[c, a, b]+L^{(i+j+k+1)}=L^{(i+j+k+1)} .
\end{aligned}
$$


Considere o $\mathbb{K}$-módulo $B(G)=\bigoplus_{i \geq 1} B_{i}(G)$. De forma abreviada, escreva $B(G)$ como $B$. Denote por $\bar{p}$ um elemento de $B$ e escreva $\bar{p}=\sum_{i \geq 1} \tilde{p}_{i}$, em que $\tilde{p}_{i} \in B_{i}$ e $\tilde{p}_{i} \neq 0$ somente para um número finito de valores de $i$. Seja $\phi: B \times B \rightarrow B$ a aplicação definida por

$$
\phi(\bar{f}, \bar{g})=\sum_{i, j \geq 1} \phi_{i j}\left(\tilde{f}_{i}, \tilde{g}_{j}\right)=\sum_{k \geq 1} \sum_{i+j=k} \phi_{i j}\left(\tilde{f}_{i}, \tilde{g}_{j}\right), \text { em que } \bar{f}=\sum_{i \geq 1} \tilde{f}_{i} \text { e } \bar{g}=\sum_{j \geq 1} \tilde{g}_{j} \text {. }
$$

Decorre imediatamente da definição que $\phi$ é uma aplicação bilinear.

Proposição 2.60. Para todos $\bar{f}, \bar{g}, \bar{h} \in B$, temos que

(i) $\phi(\bar{f}, \bar{f})=0$;

(ii) $\phi(\phi(\bar{f}, \bar{g}), \bar{h})+\phi(\phi(\bar{g}, \bar{h}), \bar{f})+\phi(\phi(\bar{h}, \bar{f}), \bar{g})=0$.

Demonstração. Escreva $\bar{f}=\sum_{i \geq 1} \tilde{f}_{i}, \bar{g}=\sum_{j \geq 1} \tilde{g}_{j}$ e $\bar{h}=\sum_{k \geq 1} \tilde{h_{k}}$. Assim,

$$
\begin{gathered}
\phi(\bar{f}, \bar{f})=\sum_{t \geq 1} \sum_{i+j=t} \phi_{i j}\left(\tilde{f}_{i}, \tilde{f}_{j}\right)=\sum_{t \geq 1} \sum_{i=1}^{t-1} \phi_{i(t-i)}\left(\tilde{f}_{i}, \tilde{f}_{t-i}\right) \\
=\sum_{t \text { impar }} \sum_{i=1}^{\frac{t-1}{2}}\left(\phi_{i(t-i)}\left(\tilde{f}_{i}, \tilde{f}_{t-i}\right)+\phi_{(t-i) i}\left(\tilde{f}_{t-i}, \tilde{f}_{t}\right)\right) \\
+\sum_{t \text { par }}\left(\sum_{i=1}^{\frac{t-2}{2}}\left(\phi_{i(t-i)}\left(\tilde{f}_{i}, \tilde{f}_{t-i}\right)+\phi_{(t-i) i}\left(\tilde{f}_{t-i}, \tilde{f}_{t}\right)\right)+\phi_{\frac{t}{2} \frac{t}{2}}\left(\tilde{f}_{\frac{t}{2}}, \tilde{f}_{\frac{t}{2}}\right)\right) .
\end{gathered}
$$

Segue então dos itens i) e ii) da proposição 2.59 que $\phi(\bar{f}, \bar{f})=0$. Agora note que

$$
\phi(\phi(\bar{f}, \bar{g}), \bar{h})=\sum_{r \geq 1} \sum_{i+j+k=r} \phi_{(i+j) k}\left(\phi_{i j}\left(\tilde{f}_{i}, \tilde{g}_{j}\right), \tilde{h}_{k}\right)
$$

e, portanto, a conclusão da proposição é uma consequência do item iii) da proposição 2.59 .

No $\mathbb{K}$-módulo $B$, considere a operação $[$,$] definida por [\bar{f}, \bar{g}]:=\phi(\bar{f}, \bar{g})$, para todos $\bar{f}, \bar{g} \in B$.

Proposição 2.61. O $\mathbb{K}$-módulo $B$ munido da operação $[$,$] é uma \mathbb{K}$-álgebra de Lie $\mathbb{N}$ graduada gerada, como álgebra, por $B_{1}$. 
Demonstração. Decorre da proposição 2.60 que $B$ é uma álgebra de Lie. Decorre da definição da operação $\phi_{i j}$ que $\left[B_{i}, B_{j}\right] \subset B_{i+j}$ e, portanto, $B$ é $\mathbb{N}$-graduada. Agora note que

$$
\left[a_{1}, a_{2}\right]+L^{(3)}=\left[a_{1}+L^{(2)}, a_{2}+L^{(2)}\right] \text {, para todos } a_{1}, a_{2} \in G
$$

Suponha então que $\left[a_{1}, a_{2}, \ldots, a_{i-1}\right]+L^{(i)}=\left[a_{1}+L^{(2)}, a_{2}+L^{(2)}, \ldots, a_{i-1}+L^{(2)}\right]$ para todos $a_{1}, a_{2}, \ldots, a_{i-1} \in G$. Assim

$$
\begin{gathered}
{\left[a_{1}, a_{2}, \ldots, a_{i-1}, a_{i}\right]+L^{(i+1)}=\left[\left[a_{1}, a_{2}, \ldots, a_{i-1}\right]+L^{(i)}, a_{i}+L^{(2)}\right]} \\
=\left[a_{1}+L^{(2)}, a_{2}+L^{(2)}, \ldots, a_{i-1}+L^{(2)}, a_{i}+L^{(2)}\right] .
\end{gathered}
$$

Disso segue que $B$ é gerada como álgebra por $B_{1}$.

Definição 2.62. A $\mathbb{K}$-álgebra graduada $B(G)$ é denominada álgebra de Lie associada a série central inferior de $G$ (dada em 2.2).

Corolário 2.63. Seja $\tilde{b} \in B_{1}$. Então $\tilde{b} \in Z(B)$ se, e somente se, $\left[\tilde{b}, B_{1}\right]=0$.

Demonstração. Um sentido é evidente e outro é consequência da álgebra $B$ ser gerada, como álgebra, por $B_{1}$.

Corolário 2.64. Seja $b \in G$. Então $b+L^{(2)} \in Z(B)$ se, e somente, se $[b, a] \in L^{(3)}$ para todo $a \in G$.

Demonstração. Temos que $\left[b+L^{(2)}, a+L^{(2)}\right]=[b, a]+L^{(3)}$, em que $a \in G$. Se $b+L^{(2)} \in$ $Z(B)$, então $[b, a] \in L^{(3)}$. Se $[b, a] \in L^{(3)}$, para todo $a \in G$, então $\left[b+L^{(2)}, B_{1}\right]=0$ e, pelo lema acima, $b+L^{(2)} \in Z(B)$.

Observação 2.65. Um caso particular, que será considerado no capítulo 3, é o que se obtém tomando $G=A^{(-)}$(a álgebra de Lie associada a uma álgebra associativa $A$ ). 


\section{Capítulo 3}

\section{Os ideais de uma álgebra associativa gerados por comutadores}

Seja $\mathbb{K}$ um anel associativo, unitário e comutativo e seja $A$ uma $\mathbb{K}$-álgebra unitária e associativa. Seja $T^{(n)}(A), n \geq 2$, o ideal bilateral em $A$ gerado pelos comutadores $\left[a_{1}, \ldots, a_{n}\right]$ em que $a_{1}, \ldots, a_{n} \in A$. Além disso, defina $T^{(1)}(A):=A$. Seja $\mathcal{E}=\left\{e_{1}, e_{2}, \ldots\right\}$ um conjunto gerador da álgebra $A$ e denote por $\mathcal{E}^{k}(k \geq 1)$ o conjunto dos elementos de $A$ da forma $e_{i_{1}} e_{i_{2}} \ldots e_{i_{k}}\left(e_{i_{j}} \in \mathcal{E}\right)$. Um dos objetivos principais deste capítulo é mostrar que

1) se $\frac{1}{6} \in \mathbb{K}$, então $T^{(n)}(A)$ é gerado como ideal bilateral pelo conjunto

$$
\left\{\left[u_{1}, \ldots, u_{n}\right] \mid u_{i} \in \mathcal{E} \cup \mathcal{E}^{2}\right\}
$$

2) se $\frac{1}{3} \in \mathbb{K}$, então $T^{(n)}(A)$ é gerado como ideal bilateral pelo conjunto

$$
\left\{\left[u_{1}, \ldots, u_{n}\right] \mid u_{i} \in \mathcal{E} \cup \mathcal{E}^{2} \cup \mathcal{E}^{3}\right\}
$$

(ver teorema 3.35)

Outro objetivo é o de apresentar um método recursivo para obter um conjunto de geradores para $T^{(n)}(\mathbb{K}\langle X\rangle$ ), como ideal bilateral em $\mathbb{K}\langle X\rangle$ (a $\mathbb{K}$-álgebra associativa unitária livre, livremente gerada pelo conjunto $X)$, a partir dos geradores de $T^{(n-2)}(\mathbb{K}\langle X\rangle)$. As seções 3.1 e 3.2 deste capítulo objetivam a demonstração desse método e, por meio dele, será demonstrado nas seções 3.3 e 3.4 os itens 1) e 2) acima citados.

Neste capítulo, para efeito de simplificação, defina $T^{(n)}:=T^{(n)}(\mathbb{K}\langle X\rangle)$. 


\subsection{Resultados auxiliares}

Ao longo desta seção, faremos uso das seguintes relações:

R1) $[a, b c]=b[a, c]+[a, b] c, a, b, c \in \mathbb{K}\langle X\rangle$,

R2) $[a b, c, d]=a[b, c, d]+[a, c, d] b+[a, d][b, c]+[a, c][b, d], a, b, c, d \in \mathbb{K}\langle X\rangle$,

em que a verificação é imediata.

Seja $U$ o ideal bilateral de $\mathbb{K}\langle X\rangle$ gerado por um conjunto não vazio $S$ e seja $W$ o ideal bilateral em $\mathbb{K}\langle X\rangle$ gerado pelo conjunto $\left\{\left[u, a_{1}, a_{2}\right] \mid u \in U, a_{1}, a_{2} \in \mathbb{K}\langle X\rangle\right\}$.

Lema 3.1. $u\left[a_{1}, a_{2}, a_{3}\right] \in W$ para todo $u \in U$ e $a_{i} \in \mathbb{K}\langle X\rangle, i=1,2,3$.

Demonstração. Segue da relação

$$
\left[a_{1}, a_{2}, u a_{3}\right]=u\left[a_{1}, a_{2}, a_{3}\right]+\left[a_{1}, a_{2}, u\right] a_{3}
$$

e da identidade de Jacobi que

$$
u\left[a_{1}, a_{2}, a_{3}\right]=\left[u a_{3}, a_{2}, a_{1}\right]-\left[u a_{3}, a_{1}, a_{2}\right]-\left[u, a_{2}, a_{1}\right] a_{3}+\left[u, a_{1}, a_{2}\right] a_{3} .
$$

Portanto, $u\left[a_{1}, a_{2}, a_{3}\right] \in W$.

Considere os polinômios $f_{1}, \ldots, f_{6} \in \mathbb{K}\langle X\rangle$ tais que

- $\quad f_{1}=f_{1}\left(x_{1}, x_{2}, x_{3}\right)=\left[x_{1}, x_{2}, x_{3}\right]$

- $f_{2}=f_{2}\left(x_{1}, x_{2}, x_{3}, x_{4}\right)=x_{1}\left[x_{2}, x_{3}, x_{4}\right]$;

- $f_{3}=f_{3}\left(x_{1}, x_{2}, x_{3}, x_{4}, x_{5}\right)=\left[x_{1}, x_{2}\right]\left[x_{3}, x_{4}, x_{5}\right]$;

- $f_{4}=f_{4}\left(x_{1}, x_{2}, x_{3}, x_{4}\right)=\left[x_{1}, x_{2}\right]\left[x_{3}, x_{4}\right]+\left[x_{1}, x_{3}\right]\left[x_{2}, x_{4}\right] ;$

- $f_{5}=f_{5}\left(x_{1}, x_{2}, x_{3}, x_{4}, x_{5}\right)=x_{1}\left(\left[x_{2}, x_{3}\right]\left[x_{4}, x_{5}\right]+\left[x_{2}, x_{4}\right]\left[x_{3}, x_{5}\right]\right)$,

em que $x_{i} \in X$. Seja $V$ o ideal bilateral de $\mathbb{K}\langle X\rangle$ gerado pelos polinômios

$$
f_{1}\left(s, x_{i_{1}}, x_{i_{2}}\right)=\left[s, x_{i_{1}}, x_{i_{2}}\right]
$$

$$
f_{2}\left(s, x_{i_{1}}, x_{i_{2}}, x_{i_{3}}\right)=s\left[x_{i_{1}}, x_{i_{2}}, x_{i_{3}}\right]
$$




$$
\begin{aligned}
& f_{3}\left(s, x_{i_{1}}, x_{i_{2}}, x_{i_{3}}, x_{i_{4}}\right)=\left[s, x_{i_{1}}\right]\left[x_{i_{2}}, x_{i_{3}}, x_{i_{4}}\right] \\
& f_{4}\left(s, x_{i_{1}}, x_{i_{2}}, x_{i_{3}}\right)=\left[s, x_{i_{1}}\right]\left[x_{i_{2}}, x_{i_{3}}\right]+\left[s, x_{i_{2}}\right]\left[x_{i_{1}}, x_{i_{3}}\right] \\
& f_{5}\left(s, x_{i_{1}}, x_{i_{2}}, x_{i_{3}}, x_{i_{4}}\right)=s\left(\left[x_{i_{1}}, x_{i_{2}}\right]\left[x_{i_{3}}, x_{i_{4}}\right]+\left[x_{i_{1}}, x_{i_{3}}\right]\left[x_{i_{2}}, x_{i_{4}}\right]\right),
\end{aligned}
$$

em que $s \in S$ e $x_{i_{j}} \in X$. Na sequência desta seção serão apresentados alguns lemas com o objetivo de mostrar que $W=V$.

Seja $I$ o ideal bilateral de $\mathbb{K}\langle X\rangle$ gerado pelos polinômios

$$
\begin{aligned}
& f_{1}\left(s, a_{1}, a_{2}\right)=\left[s, a_{1}, a_{2}\right] ; \\
& f_{2}\left(s, a_{1}, a_{2}, a_{3}\right)=s\left[a_{1}, a_{2}, a_{3}\right] ; \\
& f_{3}\left(s, a_{1}, a_{2}, a_{3}, a_{4}\right)=\left[s, a_{1}\right]\left[a_{2}, a_{3}, a_{4}\right] ; \\
& f_{4}\left(s, a_{1}, a_{2}, a_{3}\right)=\left[s, a_{1}\right]\left[a_{2}, a_{3}\right]+\left[s, a_{2}\right]\left[a_{1}, a_{3}\right] ; \\
& f_{5}\left(s, a_{1}, a_{2}, a_{3}, a_{4}\right)=s\left(\left[a_{1}, a_{2}\right]\left[a_{3}, a_{4}\right]+\left[a_{1}, a_{3}\right]\left[a_{2}, a_{4}\right]\right),
\end{aligned}
$$

em que $s \in S$ e $a_{i} \in \mathbb{K}\langle X\rangle$ são monômios. Note que os polinômios dos tipos (1) - (5) estão em $I$ e, portanto, $V \subset I$.

Lema 3.2. $W=I$.

Demonstração. Em primeiro lugar, vamos mostrar que $I \subset W$. Para tanto, é suficiente mostrar que os polinômios dos tipos (6) - (10) pertencem a $W$. Temos que os polinômios do tipo (6) pertencem a $W$ pela definição de $W$ e os polinômios dos tipos (7) e (8) pertencem a $W$ pelo lema 3.1. Para mostrar que os polinômios dos tipos (9) e (10) estão em $W$ usamos relação R2 dada acima. Assim

$$
\begin{gathered}
{\left[s, a_{1}\right]\left[a_{2}, a_{3}\right]+\left[s, a_{2}\right]\left[a_{1}, a_{3}\right]=-\left[s, a_{1}\right]\left[a_{3}, a_{2}\right]-\left[s, a_{2}\right]\left[a_{3}, a_{1}\right]} \\
=-\left[s a_{3}, a_{1}, a_{2}\right]+s\left[a_{3}, a_{1}, a_{2}\right]+\left[s, a_{1}, a_{2}\right] a_{3},
\end{gathered}
$$

e uma vez que os polinômios $\left[s a_{3}, a_{1}, a_{2}\right]$ e $\left[s, a_{1}, a_{2}\right] a_{3}$ estão em $W$, pela definição de $W$, e $s\left[a_{3}, a_{1}, a_{2}\right]$ está em $W$ pelo lema 3.1, então os polinômios do tipo (9) pertencem a $W$. Quanto aos polinômios (10) temos que

$$
s\left(\left[a_{1}, a_{2}\right]\left[a_{3}, a_{4}\right]+\left[a_{1}, a_{3}\right]\left[a_{2}, a_{4}\right]\right)=-s\left(\left[a_{1}, a_{2}\right]\left[a_{4}, a_{3}\right]+\left[a_{1}, a_{3}\right]\left[a_{4}, a_{2}\right]\right)
$$




$$
=-s\left[a_{1} a_{4}, a_{2}, a_{3}\right]+s a_{1}\left[a_{4}, a_{2}, a_{3}\right]+s\left[a_{1}, a_{2}, a_{3}\right] a_{4},
$$

e uma vez que os polinômios $s\left[a_{1} a_{4}, a_{2}, a_{3}\right], s a_{1}\left[a_{4}, a_{2}, a_{3}\right]$ e $s\left[a_{1}, a_{2}, a_{3}\right] a_{4}$ pertencem a $W$ pelo lema 3.1, segue que os polinômios do tipo (10) pertencem a $W$.

Para verificar que $W \subset I$ é suficiente mostrar que $\left[b s c, a_{1}, a_{2}\right] \in I$ para todos $s \in S$ e $b, c, a_{1}, a_{2} \in \mathbb{K}\langle X\rangle$. Temos

$$
\begin{gathered}
{\left[b s c, a_{1}, a_{2}\right]=\left[b s, a_{1}\right]\left[c, a_{2}\right]+\left[b s, a_{2}\right]\left[c, a_{1}\right]+b s\left[c, a_{1}, a_{2}\right]+\left[b s, a_{1}, a_{2}\right] c} \\
=b\left[s, a_{1}\right]\left[c, a_{2}\right]+\left[b, a_{1}\right] s\left[c, a_{2}\right]+b\left[s, a_{2}\right]\left[c, a_{1}\right]+\left[b, a_{2}\right] s\left[c, a_{1}\right]+b s\left[c, a_{1}, a_{2}\right] \\
+\left[b, a_{1}\right]\left[s, a_{2}\right] c+\left[b, a_{2}\right]\left[s, a_{1}\right] c+\left[b, a_{1}, a_{2}\right] s c+b\left[s, a_{1}, a_{2}\right] c \\
=\left(b\left[s, a_{1}\right]\left[c, a_{2}\right]+b\left[s, a_{2}\right]\left[c, a_{1}\right]\right)+\left(\left[b, a_{1}\right] s\left[c, a_{2}\right]+\left[b, a_{2}\right] s\left[c, a_{1}\right]\right) \\
+b s\left[c, a_{1}, a_{2}\right]+\left(\left[b, a_{1}\right]\left[s, a_{2}\right] c+\left[b, a_{2}\right]\left[s, a_{1}\right] c\right)+\left[b, a_{1}, a_{2}\right] s c+b\left[s, a_{1}, a_{2}\right] c \\
=\left(-b\left[s, a_{1}\right]\left[a_{2}, c\right]-b\left[s, a_{2}\right]\left[a_{1}, c\right]\right)+\left(-s\left[b, a_{1}\right]\left[a_{2}, c\right]+\left[b, a_{1}, s\right]\left[c, a_{2}\right]-s\left[b, a_{2}\right]\left[a_{1}, c\right]+\left[b, a_{2}, s\right]\left[c, a_{1}\right]\right) \\
+b s\left[c, a_{1}, a_{2}\right]+\left(-\left[s, a_{2}\right]\left[a_{1}, b\right] c+\left[s, a_{2},\left[a_{1}, b\right]\right] c-\left[s, a_{1}\right]\left[a_{2}, b\right] c+\left[s, a_{1},\left[a_{2}, b\right]\right] c\right) \\
+\left(s\left[b, a_{1}, a_{2}\right] c+\left[b, a_{1}, a_{2}, s\right] c\right)+b\left[s, a_{1}, a_{2}\right] c \\
=-b\left(\left[s, a_{1}\right]\left[a_{2}, c\right]+\left[s, a_{2}\right]\left[a_{1}, c\right]\right)-s\left(\left[b, a_{1}\right]\left[a_{2}, c\right]+\left[b, a_{2}\right]\left[a_{1}, c\right]\right) \\
-\left[s,\left[b, a_{1}\right]\right]\left[c, a_{2}\right]-\left[s,\left[b, a_{2}\right]\right]\left[c, a_{1}\right]+b s\left[c, a_{1}, a_{2}\right] \\
-\left(\left[s, a_{2}\right]\left[a_{1}, b\right]+\left[s, a_{1}\right]\left[a_{2}, b\right]\right) c+\left[s, a_{2},\left[a_{1}, b\right]\right] c+\left[s, a_{1},\left[a_{2}, b\right]\right] c \\
+s\left[b, a_{1}, a_{2}\right] c+\left[s, a_{2},\left[b, a_{1}\right]\right] c-\left[s,\left[b, a_{1}\right], a_{2}\right] c+b\left[s, a_{1}, a_{2}\right] c .
\end{gathered}
$$

Uma vez que os polinômios

$$
\begin{gathered}
{\left[s, a_{1}\right]\left[a_{2}, c\right]+\left[s, a_{2}\right]\left[a_{1}, c\right], s\left(\left[b, a_{1}\right]\left[a_{2}, c\right]+\left[b, a_{2}\right]\left[a_{1}, c\right]\right),\left[s,\left[b, a_{1}\right]\right],\left[s,\left[b, a_{2}\right]\right],} \\
s\left[c, a_{1}, a_{2}\right],\left[s, a_{2}\right]\left[a_{1}, b\right]+\left[s, a_{1}\right]\left[a_{2}, b\right],\left[s, a_{2},\left[a_{1}, b\right]\right] c,\left[s, a_{1},\left[a_{2}, b\right]\right] \\
s\left[b, a_{1}, a_{2}\right],\left[s, a_{2},\left[b, a_{1}\right]\right],\left[s,\left[b, a_{1}\right], a_{2}\right],\left[s, a_{1}, a_{2}\right]
\end{gathered}
$$

estão em $I$, temos que $\left[b s c, a_{1}, a_{2}\right]$ também está em $I$. 
Considere a aplicação $\phi:\{1,2,3,4,5\} \longrightarrow\{2,3,4\}$ tal que $\phi(1)=2, \phi(2)=\phi(4)=3$ e $\phi(3)=\phi(5)=4$. Seja $g$ um polinômio de um dos tipos (6) - (10). Então $g=$ $f_{i}\left(s, a_{1}, \ldots, a_{\phi(i)}\right)$, em que $i \in\{1,2,3,4,5\}$ e $a_{j} \in \mathbb{K}\langle X\rangle$ são monômios, $j=1, \ldots, \phi(i)$. Defina $m(g):=\operatorname{deg} a_{1}+\ldots+\operatorname{deg} a_{\phi(i)}$. Por exemplo, $m\left(\left[s, x_{1} x_{2} x_{1}, x_{3} x_{1}\right]\right)=5$. Dado um inteiro $q \geq 3$, seja $G_{q}$ o conjunto de todos os polinômios $g$ de um dos tipos (6) - (10) tais que $m(g)<q$. Assim, por exemplo,

$G_{4}=\{\underbrace{\left[s, x_{i}, x_{j}\right],\left[s, x_{i}, x_{j} x_{k}\right],\left[s, x_{i} x_{j}, x_{k}\right]}_{\text {tipo (6) }}, \underbrace{s\left[x_{i}, x_{j}, x_{k}\right]}_{\text {tipo (7) }}, \underbrace{\left[s, x_{i}\right]\left[x_{j}, x_{k}\right]+\left[s, x_{j}\right]\left[x_{i}, x_{k}\right]}_{\text {tipo (9) }} \mid s \in S\}$.

Lema 3.3. $G_{5} \subset V$.

Demonstração. Seja $g$ um polinômio de um dos tipos (6) - (10) e seja $s \in S$. considere os casos abaixo

1) $m(g)=2$

Nesse caso, $g$ necessariamente é do tipo (6) e tem a forma $\left[s, x_{i}, x_{j}\right]$. Portanto, é do tipo (1).

2) $m(g)=3$. Temos os seguintes subcasos:

2.1) $g$ é do tipo (6) da forma $\left[s, x_{i}, x_{j} x_{k}\right]$.

Uma vez que

$$
g=x_{j}\left[s, x_{i}, x_{k}\right]+\left[s, x_{i}, x_{j}\right] x_{k},
$$

e os polinômios $\left[s, x_{i}, x_{k}\right]$ e $\left[s, x_{i}, x_{j}\right]$ são do tipo (1), então $g \in V$.

2.2) $g$ é do tipo (6) da forma $\left[s, x_{i} x_{j}, x_{k}\right]$.

Uma vez que

$$
\begin{gathered}
g=x_{i}\left[s, x_{j}, x_{k}\right]+\left[x_{i}, x_{k}\right]\left[s, x_{j}\right]+\left[s, x_{i}\right]\left[x_{j}, x_{k}\right]+\left[s, x_{i}, x_{k}\right] x_{j} \\
=x_{i}\left[s, x_{j}, x_{k}\right]+\left[s, x_{j}\right]\left[x_{i}, x_{k}\right]+\left[s, x_{i}\right]\left[x_{j}, x_{k}\right]-\left[\left[s, x_{j}\right],\left[x_{i}, x_{k}\right]\right]+\left[s, x_{i}, x_{k}\right] x_{j} \\
=x_{i}\left[s, x_{j}, x_{k}\right]+\left[s, x_{j}\right]\left[x_{i}, x_{k}\right]+\left[s, x_{i}\right]\left[x_{j}, x_{k}\right] \\
-\left[s, x_{j}, x_{i} x_{k}\right]+\left[s, x_{j}, x_{k} x_{i}\right]+\left[s, x_{i}, x_{k}\right] x_{j},
\end{gathered}
$$


os polinômios $\left[s, x_{j}, x_{k}\right]$ e $\left[s, x_{i}, x_{k}\right]$ são do tipo (1), o polinômio $\left[s, x_{j}\right]\left[x_{i}, x_{k}\right]+$ $\left[s, x_{i}\right]\left[x_{j}, x_{k}\right]$ é do tipo (4) e os polinômios $\left[s, x_{j}, x_{i} x_{k}\right]$ e $\left[s, x_{j}, x_{k} x_{i}\right]$ estão em $V$ pelo item 2.1, então $g \in V$.

2.3) $g$ é do tipo (7).

Nesse caso, necessariamente, $g$ é da forma $s\left[x_{i}, x_{j}, x_{k}\right]$ e, portanto, é do tipo (2).

2.4) $g$ é do tipo (9).

Nesse caso, necessariamente, $g$ é da forma $\left[s, x_{i}\right]\left[x_{j}, x_{k}\right]+\left[s, x_{j}\right]\left[x_{i}, x_{k}\right]$ e, portanto, é do tipo (4).

3) $m(g)=4$. Temos os seguintes subcasos:

3.1) $g$ é do tipo (9) da forma $\left[s, x_{i} x_{j}\right]\left[x_{k}, x_{l}\right]+\left[s, x_{k}\right]\left[x_{i} x_{j}, x_{l}\right]$.

Uma vez que

$$
\begin{gathered}
g=x_{i}\left[s, x_{j}\right]\left[x_{k}, x_{l}\right]+\left[s, x_{i}\right] x_{j}\left[x_{k}, x_{l}\right]+\left[s, x_{k}\right] x_{i}\left[x_{j}, x_{l}\right]+\left[s, x_{k}\right]\left[x_{i}, x_{l}\right] x_{j} \\
=x_{i}\left[s, x_{j}\right]\left[x_{k}, x_{l}\right]+\left[s, x_{i}\right]\left[x_{k}, x_{l}\right] x_{j}-\left[s, x_{i}\right]\left[x_{k}, x_{l}, x_{j}\right] \\
+x_{i}\left[s, x_{k}\right]\left[x_{j}, x_{l}\right]+\left[s, x_{k}, x_{i}\right]\left[x_{j}, x_{l}\right]+\left[s, x_{k}\right]\left[x_{i}, x_{l}\right] x_{j} \\
=x_{i}\left(\left[s, x_{j}\right]\left[x_{k}, x_{l}\right]+\left[s, x_{k}\right]\left[x_{j}, x_{l}\right]\right)+\left(\left[s, x_{i}\right]\left[x_{k}, x_{l}\right]+\left[s, x_{k}\right]\left[x_{i}, x_{l}\right]\right) x_{j} \\
-\left[s, x_{i}\right]\left[x_{k}, x_{l}, x_{j}\right]+\left[s, x_{k}, x_{i}\right]\left[x_{j}, x_{l}\right]
\end{gathered}
$$

e os polinômios $\left[s, x_{j}\right]\left[x_{k}, x_{l}\right]+\left[s, x_{k}\right]\left[x_{j}, x_{l}\right]$ e $\left[s, x_{i}\right]\left[x_{k}, x_{l}\right]+\left[s, x_{k}\right]\left[x_{i}, x_{l}\right]$ são do tipo (4), o polinômio $\left[s, x_{i}\right]\left[x_{k}, x_{l}, x_{j}\right]$ é do tipo (3) e o polinômio $\left[s, x_{k}, x_{i}\right]$ é do tipo (1), então $g \in V$.

3.2) $g$ é do tipo (9) da forma $\left[s, x_{i}\right]\left[x_{j}, x_{k} x_{l}\right]+\left[s, x_{j}\right]\left[x_{i}, x_{k} x_{l}\right]$.

Uma vez que

$$
\begin{gathered}
g=\left[s, x_{i}\right] x_{k}\left[x_{j}, x_{l}\right]+\left[s, x_{i}\right]\left[x_{j}, x_{k}\right] x_{l}+\left[s, x_{j}\right] x_{k}\left[x_{i}, x_{l}\right]+\left[s, x_{j}\right]\left[x_{i}, x_{k}\right] x_{l} \\
=x_{k}\left(\left[s, x_{i}\right]\left[x_{j}, x_{l}\right]+\left[s, x_{j}\right]\left[x_{i}, x_{l}\right]\right)+\left[s, x_{i}, x_{k}\right]\left[x_{j}, x_{l}\right] \\
+\left[s, x_{j}, x_{k}\right]\left[x_{i}, x_{l}\right]+\left(\left[s, x_{i}\right]\left[x_{j}, x_{k}\right]+\left[s, x_{j}\right]\left[x_{i}, x_{k}\right]\right) x_{l}
\end{gathered}
$$


e os polinômios $\left[s, x_{i}\right]\left[x_{j}, x_{l}\right]+\left[s, x_{j}\right]\left[x_{i}, x_{l}\right]$ e $\left[s, x_{i}\right]\left[x_{j}, x_{k}\right]+\left[s, x_{j}\right]\left[x_{i}, x_{k}\right]$ são do tipo (4) e os polinômios $\left[s, x_{i}, x_{k}\right]$ e $\left[s, x_{j}, x_{k}\right]$ são do tipo (1), então $g \in V$.

3.3) $g$ é do tipo (6) da forma $\left[s, x_{i} x_{j}, x_{k} x_{l}\right]$.

Uma vez que

$$
g=\left[s, x_{i} x_{j}, x_{k}\right] x_{l}+x_{k}\left[s, x_{i} x_{j}, x_{l}\right]
$$

e os polinômios $\left[s, x_{i} x_{j}, x_{k}\right]$ e $\left[s, x_{i} x_{j}, x_{l}\right]$ estão em $V$ pelo item 2.2 , então $g \in V$.

3.4) $g$ é do tipo (6) da forma $\left[s, x_{i} x_{j} x_{k}, x_{l}\right]$.

Uma vez que

$$
\begin{gathered}
g=x_{i}\left[s, x_{j} x_{k}, x_{l}\right]+\left[x_{i}, x_{l}\right]\left[s, x_{j} x_{k}\right]+\left[s, x_{i}\right]\left[x_{j} x_{k}, x_{l}\right]+\left[s, x_{i}, x_{l}\right] x_{j} x_{k} \\
=x_{i}\left[s, x_{j} x_{k}, x_{l}\right]+\left[s, x_{j} x_{k}\right]\left[x_{i}, x_{l}\right]+\left[s, x_{i}\right]\left[x_{j} x_{k}, x_{l}\right] \\
-\left[s, x_{j} x_{k},\left[x_{i}, x_{l}\right]\right]+\left[s, x_{i}, x_{l}\right] x_{j} x_{k} \\
=x_{i}\left[s, x_{j} x_{k}, x_{l}\right]+\left[s, x_{j} x_{k}\right]\left[x_{i}, x_{l}\right]+\left[s, x_{i}\right]\left[x_{j} x_{k}, x_{l}\right] \\
-\left[s, x_{j} x_{k}, x_{i} x_{l}\right]+\left[s, x_{j} x_{k}, x_{l} x_{i}\right]+\left[s, x_{i}, x_{l}\right] x_{j} x_{k},
\end{gathered}
$$

o polinômio $\left[s, x_{j} x_{k}, x_{l}\right]$ está em $V$ pelo item $2.2,\left[s, x_{j} x_{k}\right]\left[x_{i}, x_{l}\right]+\left[s, x_{i}\right]\left[x_{j} x_{k}, x_{l}\right]$ está em $V$ pelo item 3.1, $\left[s, x_{j} x_{k}, x_{i} x_{l}\right]$ e $\left[s, x_{j} x_{k}, x_{l} x_{i}\right]$ estão em $V$ pelo item 3.3 e o $\left[s, x_{i}, x_{l}\right]$ é do tipo (1), então $g \in V$.

3.5) $g$ é do tipo (6) da forma $\left[s, x_{i}, x_{j} x_{k} x_{l}\right]$.

Uma vez que

$$
g=x_{j}\left[s, x_{i}, x_{k} x_{l}\right]+\left[s, x_{i}, x_{j}\right] x_{k} x_{l},
$$

o polinômio $\left[s, x_{i}, x_{k} x_{l}\right]$ está em $V$ pelo caso 2.1 e o polinômio $\left[s, x_{i}, x_{j}\right]$ é do tipo (1), então $g \in V$.

3.6) $g$ é do tipo (7) da forma $s\left[x_{i} x_{j}, x_{k}, x_{l}\right]$.

Uma vez que

$$
\begin{gathered}
g=s x_{i}\left[x_{j}, x_{k}, x_{l}\right]+s\left[x_{i}, x_{k}\right]\left[x_{j}, x_{l}\right]+s\left[x_{i}, x_{l}\right]\left[x_{j}, x_{k}\right]+s\left[x_{i}, x_{k}, x_{l}\right] x_{j} \\
=x_{i} s\left[x_{j}, x_{k}, x_{l}\right]+\left[s, x_{i}\right]\left[x_{j}, x_{k}, x_{l}\right]
\end{gathered}
$$




$$
-s\left(\left[x_{i}, x_{k}\right]\left[x_{l}, x_{j}\right]+\left[x_{i}, x_{l}\right]\left[x_{k}, x_{j}\right]\right)+s\left[x_{i}, x_{k}, x_{l}\right] x_{j},
$$

os polinômios $s\left[x_{j}, x_{k}, x_{l}\right]$ e $s\left[x_{i}, x_{k}, x_{l}\right]$ são do tipo $(2),\left[s, x_{i}\right]\left[x_{j}, x_{k}, x_{l}\right]$ é do tipo $(3)$ e $s\left(\left[x_{i}, x_{k}\right]\left[x_{l}, x_{j}\right]+\left[x_{i}, x_{l}\right]\left[x_{k}, x_{j}\right]\right)$ é do tipo (5), então $g \in V$.

3.7) $g$ é do tipo (7) da forma $s\left[x_{i}, x_{j}, x_{k} x_{l}\right]$.

Uma vez que $g=\left[x_{k} x_{l}, x_{j}, x_{i}\right]-\left[x_{k} x_{l}, x_{i}, x_{j}\right]$, então $g \in V$ pelo caso 3.6.

3.8) $g$ é do tipo (8).

Nesse caso, necessariamente, $g$ é da forma $\left[s, x_{i}\right]\left[x_{j}, x_{k}, x_{l}\right]$ e, portanto, é do tipo (3).

3.9) $g$ é do tipo (10).

Nesse caso, necessariamente, $g$ é da forma $s\left(\left[x_{i}, x_{j}\right]\left[x_{k}, x_{l}\right]+\left[x_{i}, x_{k}\right]\left[x_{j}, x_{l}\right]\right)$ e, portanto, é do tipo (5).

Lema 3.4. Sejam $b_{1}, b_{2}, b_{3}, b_{4}, b_{5} \in \mathbb{K}\langle X\rangle$ monômios tais que $\operatorname{deg} b_{i} \geq 1(i=1, \ldots, 5)$ e $\sum_{i=1}^{5} \operatorname{deg} b_{i}=q$. Suponha que $G_{q} \subset V$. Então

i) $\left[s, b_{1}\right]\left[b_{2}, b_{3},\left[b_{4}, b_{5}\right]\right] \in V$;

ii) $\left[s, b_{1}\right]\left[b_{2}, b_{3}\right]\left[b_{4}, b_{5}\right]-\left[s, b_{1}\right]\left[b_{4}, b_{5}\right]\left[b_{2}, b_{3}\right] \in V$;

iii) $\left[s, b_{1}\right]\left[b_{2}, b_{3}\right]\left[b_{4}, b_{5}\right]+\left[s, b_{2}\right]\left[b_{1}, b_{3}\right]\left[b_{4}, b_{5}\right] \in V$;

iv) $\left[s, b_{1}\right]\left(\left[b_{2}, b_{3}\right]\left[b_{4}, b_{5}\right]+\left[b_{2}, b_{4}\right]\left[b_{3}, b_{5}\right]\right) \in V$;

v) $s b_{1}\left(\left[b_{2}, b_{3}\right]\left[b_{4}, b_{5}\right]+\left[b_{2}, b_{4}\right]\left[b_{3}, b_{5}\right]\right) \in V$

em que $s \in S$.

Demonstração.

i) Temos

$$
\begin{aligned}
& {\left[s, b_{1}\right]\left[b_{2}, b_{3}, b_{4} b_{5}\right]=\left[s, b_{1}\right] b_{4}\left[b_{2}, b_{3}, b_{5}\right]+\left[s, b_{1}\right]\left[b_{2}, b_{3}, b_{4}\right] b_{5} } \\
= & b_{4}\left[s, b_{1}\right]\left[b_{2}, b_{3}, b_{5}\right]+\left[s, b_{1}, b_{4}\right]\left[b_{2}, b_{3}, b_{5}\right]+\left[s, b_{1}\right]\left[b_{2}, b_{3}, b_{4}\right] b_{5},
\end{aligned}
$$


e uma vez que $\left[s, b_{1}\right]\left[b_{2}, b_{3}, b_{5}\right],\left[s, b_{1}, b_{4}\right],\left[s, b_{1}\right]\left[b_{2}, b_{3}, b_{4}\right] \in G_{q}$, então $\left[s, b_{1}\right]\left[b_{2}, b_{3}, b_{4} b_{5}\right] \in V$. Logo, $\left[s, b_{1}\right]\left[b_{2}, b_{3},\left[b_{4}, b_{5}\right]\right] \in V$.

ii) Temos

$$
\left[s, b_{1}\right]\left[b_{2}, b_{3}\right]\left[b_{4}, b_{5}\right]=\left[s, b_{1}\right]\left[\left[b_{2}, b_{3}\right],\left[b_{4}, b_{5}\right]\right]+\left[s, b_{1}\right]\left[b_{4}, b_{5}\right]\left[b_{2}, b_{3}\right],
$$

e, portanto, o resultado segue do item anterior.

iii) Uma vez que, por hipótese, $\left[s, b_{1}\right]\left[b_{2}, b_{3}\right]+\left[s, b_{2}\right]\left[b_{1}, b_{3}\right] \in V$, então

$$
\left[s, b_{1}\right]\left[b_{2}, b_{3}\right]\left[b_{4}, b_{5}\right]+\left[s, b_{2}\right]\left[b_{1}, b_{3}\right]\left[b_{4}, b_{5}\right] \in V .
$$

iv) Segue dos itens anteriores que

$$
\begin{aligned}
& {\left[s, b_{1}\right]\left[b_{2}, b_{3}\right]\left[b_{4}, b_{5}\right]+V=-\left[s, b_{2}\right]\left[b_{1}, b_{3}\right]\left[b_{4}, b_{5}\right]+V=-\left[s, b_{2}\right]\left[b_{4}, b_{5}\right]\left[b_{1}, b_{3}\right]+V } \\
= & {\left[s, b_{2}\right]\left[b_{5}, b_{4}\right]\left[b_{1}, b_{3}\right]+V=-\left[s, b_{5}\right]\left[b_{2}, b_{4}\right]\left[b_{1}, b_{3}\right]+V=-\left[s, b_{5}\right]\left[b_{1}, b_{3}\right]\left[b_{2}, b_{4}\right]+V } \\
= & {\left[s, b_{1}\right]\left[b_{5}, b_{3}\right]\left[b_{2}, b_{4}\right]+V=\left[s, b_{1}\right]\left[b_{2}, b_{4}\right]\left[b_{5}, b_{3}\right]+V=-\left[s, b_{1}\right]\left[b_{2}, b_{4}\right]\left[b_{3}, b_{5}\right]+V . }
\end{aligned}
$$

Portanto, $\left[s, b_{1}\right]\left[b_{2}, b_{3}\right]\left[b_{4}, b_{5}\right]+\left[s, b_{1}\right]\left[b_{2}, b_{4}\right]\left[b_{3}, b_{5}\right] \in V$.

v) Uma vez que

$$
\begin{gathered}
s b_{1}\left(\left[b_{2}, b_{3}\right]\left[b_{4}, b_{5}\right]+\left[b_{2}, b_{4}\right]\left[b_{3}, b_{5}\right]\right) \\
=b_{1}\left(s\left(\left[b_{2}, b_{3}\right]\left[b_{4}, b_{5}\right]+\left[b_{2}, b_{4}\right]\left[b_{3}, b_{5}\right]\right)\right)+\left[s, b_{1}\right]\left(\left[b_{2}, b_{3}\right]\left[b_{4}, b_{5}\right]+\left[b_{2}, b_{4}\right]\left[b_{3}, b_{5}\right]\right),
\end{gathered}
$$

o resultado segue do fato de que $\left[s, b_{1}\right]\left(\left[b_{2}, b_{3}\right]\left[b_{4}, b_{5}\right]+\left[b_{2}, b_{4}\right]\left[b_{3}, b_{5}\right]\right) \in V$ (pelo item iv) e $s\left(\left[b_{2}, b_{3}\right]\left[b_{4}, b_{5}\right]+\left[b_{2}, b_{4}\right]\left[b_{3}, b_{5}\right]\right) \in G_{q} \subset V$.

\section{Lema 3.5. $W=V$.}

Demonstração. De acordo com o lema 3.2 é suficiente mostrar que $I=V$. Como já foi observado, a inclusão $V \subset I$ é imediata. Para mostrar que $I \subset V$ é suficiente mostrar que os polinômios dos tipos (6) - (10) estão em $V$. Seja $g$ um polinômio de um dos tipos (6) - (10) tal que $m(g)=q$. A prova será feita por indução sobre $q$. Sabemos que se $q<5$, então $g \in V$ (lema 3.3). Desta forma, a base da indução está dada. Suponha que $q \geq 5$ e que $G_{q} \subset V$. A demonstração será dividida em 5 casos, para cada um dos tipos $(6)-(10)$. 
1을. $g$ é do tipo (10),

$$
g=s\left(\left[a_{1}, a_{2}\right]\left[a_{3}, a_{4}\right]+\left[a_{1}, a_{3}\right]\left[a_{2}, a_{4}\right]\right) .
$$

Temos que, para algum $j(1 \leq j \leq 4), a_{j}=b c \operatorname{com} 1 \leq \operatorname{deg} b, \operatorname{deg} c<\operatorname{deg} a_{j}$. Suponha que $a_{1}=b c$. Assim,

$$
\begin{gathered}
s\left(\left[b c, a_{2}\right]\left[a_{3}, a_{4}\right]+\left[b c, a_{3}\right]\left[a_{2}, a_{4}\right]\right) \\
=s b\left(\left[c, a_{2}\right]\left[a_{3}, a_{4}\right]+\left[c, a_{3}\right]\left[a_{2}, a_{4}\right]\right)+s\left(\left[b, a_{2}\right] c\left[a_{3}, a_{4}\right]+\left[b, a_{3}\right] c\left[a_{2}, a_{4}\right]\right) \\
=s b\left(\left[c, a_{2}\right]\left[a_{3}, a_{4}\right]+\left[c, a_{3}\right]\left[a_{2}, a_{4}\right]\right)+s c\left(\left[b, a_{2}\right]\left[a_{3}, a_{4}\right]+\left[b, a_{3}\right]\left[a_{2}, a_{4}\right]\right) \\
+\left(s\left[b, a_{2}, c\right]\right)\left[a_{3}, a_{4}\right]+\left(s\left(\left[b, a_{3}, c\right]\right)\left[a_{2}, a_{4}\right] .\right.
\end{gathered}
$$

Uma vez que $s\left[b, a_{2}, c\right], s\left[b, a_{3}, c\right] \in G_{q} \subset V$ e

$$
s b\left(\left[c, a_{2}\right]\left[a_{3}, a_{4}\right]+\left[c, a_{3}\right]\left[a_{2}, a_{4}\right]\right), s c\left(\left[b, a_{2}\right]\left[a_{3}, a_{4}\right]+\left[b, a_{3}\right]\left[a_{2}, a_{4}\right]\right) \in V
$$

pelo item v do lema 3.4, temos que $g \in V$.

Suponha que $a_{2}=b c$. Temos

$$
\begin{gathered}
s\left(\left[a_{1}, b c\right]\left[a_{3}, a_{4}\right]+\left[a_{1}, a_{3}\right]\left[b c, a_{4}\right]\right) \\
=s\left(b\left[a_{1}, c\right]\left[a_{3}, a_{4}\right]+\left[a_{1}, b\right] c\left[a_{3}, a_{4}\right]+\left[a_{1}, a_{3}\right] b\left[c, a_{4}\right]+\left[a_{1}, a_{3}\right]\left[b, a_{4}\right] c\right) \\
=s\left(b\left[a_{1}, c\right]\left[a_{3}, a_{4}\right]+\left[a_{1}, a_{3}\right] b\left[c, a_{4}\right]\right)+s\left(\left[a_{1}, a_{3}\right]\left[b, a_{4}\right] c+\left[a_{1}, b\right] c\left[a_{3}, a_{4}\right]\right) \\
=s b\left(\left[a_{1}, c\right]\left[a_{3}, a_{4}\right]+\left[a_{1}, a_{3}\right]\left[c, a_{4}\right]\right)+\left(s\left[a_{1}, a_{3}, b\right]\right)\left[c, a_{4}\right]+\left(s\left(\left[a_{1}, a_{3}\right]\left[b, a_{4}\right]+\left[a_{1}, b\right]\left[a_{3}, a_{4}\right]\right)\right) c \\
+s\left[a_{1}, b\right]\left[c,\left[a_{3}, a_{4}\right]\right] \\
=s b\left(\left[a_{1}, c\right]\left[a_{3}, a_{4}\right]+\left[a_{1}, a_{3}\right]\left[c, a_{4}\right]\right)+\left(s\left[a_{1}, a_{3}, b\right]\right)\left[c, a_{4}\right]+\left(s\left(\left[a_{1}, a_{3}\right]\left[b, a_{4}\right]+\left[a_{1}, b\right]\left[a_{3}, a_{4}\right]\right)\right) c \\
+\left(\left[s,\left[a_{1}, b\right]\right]\right)\left[c,\left[a_{3}, a_{4}\right]\right]+\left[a_{1}, b\right]\left(s\left[c,\left[a_{3}, a_{4}\right]\right]\right) \\
=s b\left(\left[a_{1}, c\right]\left[a_{3}, a_{4}\right]+\left[a_{1}, a_{3}\right]\left[c, a_{4}\right]\right)+\left(s\left[a_{1}, a_{3}, b\right]\right)\left[c, a_{4}\right]+\left(s\left(\left[a_{1}, a_{3}\right]\left[b, a_{4}\right]+\left[a_{1}, b\right]\left[a_{3}, a_{4}\right]\right)\right) c \\
+\left(\left[s, a_{1}, b\right]-\left[s, b, a_{1}\right]\right)\left[c,\left[a_{3}, a_{4}\right]\right]+\left[a_{1}, b\right]\left(s\left[c, a_{3}, a_{4}\right]-s\left[c, a_{4}, a_{3}\right]\right) .
\end{gathered}
$$

Uma vez que

$$
\begin{gathered}
s\left[a_{1}, a_{3}, b\right], s\left(\left[a_{1}, a_{3}\right]\left[b, a_{4}\right]+\left[a_{1}, b\right]\left[a_{3}, a_{4}\right]\right) \\
{\left[s, a_{1}, b\right],\left[s, b, a_{1}\right], s\left[c, a_{3}, a_{4}\right], s\left[c, a_{4}, a_{3}\right] \in G_{q} \subset V}
\end{gathered}
$$


e $s b\left(\left[a_{1}, c\right]\left[a_{3}, a_{4}\right]+\left[a_{1}, a_{3}\right]\left[c, a_{4}\right]\right) \in V$ pelo item v do lema 3.4, temos que $g \in V$.

Por fim, suponha que $a_{4}=b c$. Temos

$$
\begin{gathered}
s\left(\left[a_{1}, a_{2}\right]\left[a_{3}, b c\right]+\left[a_{1}, a_{3}\right]\left[a_{2}, b c\right]\right) \\
=s\left(\left[a_{1}, a_{2}\right] b\left[a_{3}, c\right]+\left[a_{1}, a_{3}\right] b\left[a_{2}, c\right]\right)+\left(s\left(\left[a_{1}, a_{2}\right]\left[a_{3}, b\right]+\left[a_{1}, a_{3}\right]\left[a_{2}, b\right]\right)\right) c \\
=s b\left(\left[a_{1}, a_{2}\right]\left[a_{3}, c\right]+\left[a_{1}, a_{3}\right]\left[a_{2}, c\right]\right)+\left(s\left[a_{1}, a_{2}, b\right]\right)\left[a_{3}, c\right] \\
+\left(s\left[a_{1}, a_{3}, b\right]\right)\left[a_{2}, c\right]+\left(s\left(\left[a_{1}, a_{2}\right]\left[a_{3}, b\right]+\left[a_{1}, a_{3}\right]\left[a_{2}, b\right]\right)\right) c .
\end{gathered}
$$

Uma vez que

$$
s\left[a_{1}, a_{2}, b\right], s\left[a_{1}, a_{3}, b\right], s\left(\left[a_{1}, a_{2}\right]\left[a_{3}, b\right]+\left[a_{1}, a_{3}\right]\left[a_{2}, b\right]\right) \in G_{q} \subset V
$$

e $s b\left(\left[a_{1}, a_{2}\right]\left[a_{3}, c\right]+\left[a_{1}, a_{3}\right]\left[a_{2}, c\right]\right) \in V$ pelo item v do lema 3.4, temos que $g \in V$.

2o Caso. $g$ é do tipo (8),

$$
g=\left[s, a_{1}\right]\left[a_{2}, a_{3}, a_{4}\right]
$$

Temos que, para algum $j(1 \leq j \leq 4), a_{j}=b c \operatorname{com} 1 \leq \operatorname{deg} b, \operatorname{deg} c<\operatorname{deg} a_{j}$. Suponha que $a_{1}=b c$. Assim,

$$
\begin{gathered}
{[s, b c]\left[a_{2}, a_{3}, a_{4}\right]=b\left([s, c]\left[a_{2}, a_{3}, a_{4}\right]\right)+[s, b] c\left[a_{2}, a_{3}, a_{4}\right]} \\
=b\left([s, c]\left[a_{2}, a_{3}, a_{4}\right]\right)+c\left([s, b]\left[a_{2}, a_{3}, a_{4}\right]\right)+([s, b, c])\left[a_{2}, a_{3}, a_{4}\right] .
\end{gathered}
$$

Uma vez que $[s, c]\left[a_{2}, a_{3}, a_{4}\right],[s, b]\left[a_{2}, a_{3}, a_{4}\right],[s, b, c] \in G_{q} \subset V$, temos $g \in V$.

Suponha que $a_{2}=b c$. Temos

$$
\begin{gathered}
{\left[s, a_{1}\right]\left[b c, a_{3}, a_{4}\right]=\left[s, a_{1}\right]\left(\left[b, a_{3}\right]\left[c, a_{4}\right]+\left[b, a_{4}\right]\left[c, a_{3}\right]+b\left[c, a_{3}, a_{4}\right]+\left[b, a_{3}, a_{4}\right] c\right)} \\
=\left[s, a_{1}\right]\left(\left[b, a_{3}\right]\left[c, a_{4}\right]+\left[b, a_{4}\right]\left[c, a_{3}\right]\right)+\left[s, a_{1}\right] b\left[c, a_{3}, a_{4}\right]+\left(\left[s, a_{1}\right]\left[b, a_{3}, a_{4}\right]\right) c \\
=-\left[s, a_{1}\right]\left(\left[b, a_{3}\right]\left[a_{4}, c\right]+\left[b, a_{4}\right]\left[a_{3}, c\right]\right)+b\left(\left[s, a_{1}\right]\left[c, a_{3}, a_{4}\right]\right) \\
+\left(\left[s, a_{1}, b\right]\right)\left[c, a_{3}, a_{4}\right]+\left(\left[s, a_{1}\right]\left[b, a_{3}, a_{4}\right]\right) c .
\end{gathered}
$$

Uma vez que $\left[s, a_{1}\right]\left[c, a_{3}, a_{4}\right],\left[s, a_{1}, b\right],\left[s, a_{1}\right]\left[b, a_{3}, a_{4}\right] \in G_{q} \subset V$ e $\left[s, a_{1}\right]\left(\left[b, a_{3}\right]\left[a_{4}, c\right]+\right.$ $\left.\left[b, a_{4}\right]\left[a_{3}, c\right]\right) \in V$, pelo item iv do lema 3.4 , temos que $g \in V$. 
Os subcasos em que $a_{3}=b c$ e $a_{4}=b c$ decorrem do subcaso anterior, respectivamente, pela anticomutatividade e pela identidade de Jacobi.

3o Caso. g é do tipo (7),

$$
g=s\left[a_{1}, a_{2}, a_{3}\right]
$$

Temos que, para algum $j(1 \leq j \leq 3), a_{j}=b c \operatorname{com} 1 \leq \operatorname{deg} b, \operatorname{deg} c<\operatorname{deg} a_{j}$. Suponha que $a_{1}=b c$. Assim,

$$
\begin{gathered}
s\left[b c, a_{2}, a_{3}\right]=s\left(\left[b, a_{2}\right]\left[c, a_{3}\right]+\left[b, a_{3}\right]\left[c, a_{2}\right]+b\left[c, a_{2}, a_{3}\right]+\left[b, a_{2}, a_{3}\right] c\right) \\
=-s\left(\left[b, a_{2}\right]\left[a_{3}, c\right]+\left[b, a_{3}\right]\left[a_{2}, c\right]\right)+s b\left[c, a_{2}, a_{3}\right]+\left(s\left[b, a_{2}, a_{3}\right]\right) c \\
=-s\left(\left[b, a_{2}\right]\left[a_{3}, c\right]+\left[b, a_{3}\right]\left[a_{2}, c\right]\right)+b\left(s\left[c, a_{2}, a_{3}\right]\right) \\
+[s, b]\left[c, a_{2}, a_{3}\right]+\left(s\left[b, a_{2}, a_{3}\right]\right) c .
\end{gathered}
$$

Uma vez que $s\left[c, a_{2}, a_{3}\right], s\left[b, a_{2}, a_{3}\right] \in G_{q} \subset V, s\left(\left[b, a_{2}\right]\left[a_{3}, c\right]+\left[b, a_{3}\right]\left[a_{2}, c\right]\right) \in V$, pelo $1^{\text {o }}$ caso, e $[s, b]\left[c, a_{2}, a_{3}\right] \in V$, pelo $2^{\circ}$ caso, temos que $g \in V$.

Os subcasos em que $a_{2}=b c$ e $a_{3}=b c$ decorrem do subcaso anterior, respectivamente, pela anticomutatividade e pela identidade de Jacobi.

4ㅜ Caso. $g$ é do tipo (9),

$$
g=\left[s, a_{1}\right]\left[a_{2}, a_{3}\right]+\left[s, a_{2}\right]\left[a_{1}, a_{3}\right]
$$

Temos que, para algum $j(1 \leq j \leq 3), a_{j}=b c \operatorname{com} 1 \leq \operatorname{deg} b, \operatorname{deg} c<\operatorname{deg} a_{j}$. Suponha que $a_{1}=b c$. Assim,

$$
\begin{aligned}
{[s, b c]\left[a_{2}, a_{3}\right]+\left[s, a_{2}\right] } & {\left[b c, a_{3}\right]=\left(b[s, c]\left[a_{2}, a_{3}\right]+\left[s, a_{2}\right] b\left[c, a_{3}\right]\right)+\left([s, b] c\left[a_{2}, a_{3}\right]+\left[s, a_{2}\right]\left[b, a_{3}\right] c\right) } \\
= & b\left([s, c]\left[a_{2}, a_{3}\right]+\left[s, a_{2}\right]\left[c, a_{3}\right]\right)+\left(\left[s, a_{2}, b\right]\right)\left[c, a_{3}\right] \\
+ & \left([s, b]\left[a_{2}, a_{3}\right]+\left[s, a_{2}\right]\left[b, a_{3}\right]\right) c+[s, b]\left[c,\left[a_{2}, a_{3}\right]\right] .
\end{aligned}
$$

Uma vez que $[s, c]\left[a_{2}, a_{3}\right]+\left[s, a_{2}\right]\left[c, a_{3}\right],\left[s, a_{2}, b\right],[s, b]\left[a_{2}, a_{3}\right]+\left[s, a_{2}\right]\left[b, a_{3}\right] \in G_{q} \subset V$ e $[s, b]\left[c,\left[a_{2}, a_{3}\right]\right] \in V$, pelo $2^{\circ}$ caso, temos que $g \in V$.

Por fim, suponha que $a_{3}=b c$. Temos

$$
\left[s, a_{1}\right]\left[a_{2}, b c\right]+\left[s, a_{2}\right]\left[a_{1}, b c\right]=\left(\left[s, a_{1}\right] b\left[a_{2}, c\right]+\left[s, a_{2}\right] b\left[a_{1}, c\right]\right)+\left(\left[s, a_{1}\right]\left[a_{2}, b\right]+\left[s, a_{2}\right]\left[a_{1}, b\right]\right) c
$$




$$
\begin{aligned}
& =b\left(\left[s, a_{1}\right]\left[a_{2}, c\right]+\left[s, a_{2}\right]\left[a_{1}, c\right]\right)+\left(\left[s, a_{1}, b\right]\right)\left[a_{2}, c\right] \\
& +\left(\left[s, a_{2}, b\right]\right)\left[a_{1}, c\right]+\left(\left[s, a_{1}\right]\left[a_{2}, b\right]+\left[s, a_{2}\right]\left[a_{1}, b\right]\right) c .
\end{aligned}
$$

Uma vez que $\left[s, a_{1}\right]\left[a_{2}, c\right]+\left[s, a_{2}\right]\left[a_{1}, c\right],\left[s, a_{1}, b\right],\left[s, a_{2}, b\right],\left[s, a_{1}\right]\left[a_{2}, b\right]+\left[s, a_{2}\right]\left[a_{1}, b\right] \in G_{q} \subset$ $V$, temos que $g \in V$.

5o Caso. $g$ é do tipo (6),

$$
g=\left[s, a_{1}, a_{2}\right]
$$

Temos que, para algum $j(1 \leq j \leq 2), a_{j}=b c \operatorname{com} 1 \leq \operatorname{deg} b, \operatorname{deg} c<\operatorname{deg} a_{j}$. Suponha que $a_{1}=b c$. Assim,

$$
\begin{gathered}
{\left[s, b c, a_{2}\right]=-\left[b c, s, a_{2}\right]=-[b, s]\left[c, a_{2}\right]-\left[b, a_{2}\right][c, s]-\left[b, s, a_{2}\right] c-b\left[c, s, a_{2}\right]} \\
=[s, b]\left[c, a_{2}\right]+\left[b, a_{2}\right][s, c]+\left(\left[s, b, a_{2}\right]\right) c+b\left(\left[s, c, a_{2}\right]\right) \\
=[s, b]\left[c, a_{2}\right]+[s, c]\left[b, a_{2}\right]-\left[s, c,\left[b, a_{2}\right]\right]+\left(\left[s, b, a_{2}\right]\right) c+b\left(\left[s, c, a_{2}\right]\right) \\
=[s, b]\left[c, a_{2}\right]+[s, c]\left[b, a_{2}\right]-\left[[s, c, b], a_{2}\right]+\left[\left[s, c, a_{2}\right], b\right]+\left(\left[s, b, a_{2}\right]\right) c+b\left(\left[s, c, a_{2}\right]\right) .
\end{gathered}
$$

Uma vez que $[s, c, b],\left[s, c, a_{2}\right],\left[s, b, a_{2}\right] \in G_{q} \subset V$ e $[s, b]\left[c, a_{2}\right]+[s, c]\left[b, a_{2}\right] \in V$, pelo $4^{\text {o }}$ caso, temos que $g \in V$.

Por fim, suponha que $a_{2}=b c$. Temos

$$
\left[s, a_{1}, b c\right]=b\left(\left[s, a_{1}, c\right]\right)+\left(\left[s, a_{1}, b\right]\right) c .
$$

Uma vez que $\left[s, a_{1}, c\right],\left[s, a_{1}, b\right] \in G_{q} \subset V$, temos que $g \in V$.

Em outras palavras, o lema 3.5 afirma que se

- $S$ é um subconjunto (não vazio) de $\mathbb{K}\langle X\rangle$,

- $U$ é o ideal bilateral de $\mathbb{K}\langle X\rangle$ gerado, como ideal bilateral, por $S$ e

- $W$ é o ideal bilateral de $\mathbb{K}\langle X\rangle$ gerado, como ideal bilateral, pelos elementos da forma $\left[u, a_{1}, a_{2}\right]$ em que $u \in U$ e $a_{1}, a_{2} \in \mathbb{K}\langle X\rangle$,

então $W$ coincide com o ideal bilateral de $\mathbb{K}\langle X\rangle$ gerado, como ideal bilateral, pelos polinômios 
(1) $\left[s, x_{i_{1}}, x_{i_{2}}\right]$;

(2) $s\left[x_{i_{1}}, x_{i_{2}}, x_{i_{3}}\right]$;

(3) $\left[s, x_{i_{1}}\right]\left[x_{i_{2}}, x_{i_{3}}, x_{i_{4}}\right]$;

(4) $\left[s, x_{i_{1}}\right]\left[x_{i_{2}}, x_{i_{3}}\right]+\left[s, x_{i_{2}}\right]\left[x_{i_{1}}, x_{i_{3}}\right]$;

(5) $s\left(\left[x_{i_{1}}, x_{i_{2}}\right]\left[x_{i_{3}}, x_{i_{4}}\right]+\left[x_{i_{1}}, x_{i_{3}}\right]\left[x_{i_{2}}, x_{i_{4}}\right]\right)$,

em que $x_{i_{j}} \in X$ e $s \in S$.

\subsection{Método recursivo para obter os geradores de $T^{(n)}$}

Sejam $a, b, u, v, w \in \mathbb{K}\langle X\rangle$. Temos

$$
\begin{aligned}
& {[a b, u, v]=[u, a][v, b]+[v, a][u, b]+[a, u, v] b+a[b, u, v] \quad \mathrm{e}} \\
& {[v, a][u, b]=[[v, a],[u, b]]+[u, b][v, a] .}
\end{aligned}
$$

Portanto,

$$
[u, a][v, b]+[u, b][v, a]=-[u, a b, v]+[[u, b],[v, a]]+[u, a, v] b+a[u, b, v]
$$

Além disso, temos

$$
\begin{gathered}
{[a b, u, v, w]=[[a b, u, v], w]=[[a, u][b, v], w]+[[a, v][b, u], w]+[a[b, u, v], w]+[[a, u, v] b, w]} \\
=[a, u][b, v, w]+[a, u, w][b, v]+[a, v][b, u, w]+[a, v, w][b, u] \\
+a[b, u, v, w]+[a, w][b, u, v]+[a, u, v][b, w]+[a, u, v, w] b \\
=-([u, a][b, v, w]+[u, b][a, v, w])+[[u, b],[a, v, w]]-([u, a, w][b, v]+[u, a, v][b, w]) \\
-([u, b, w][a, v]+[u, b, v][a, w])+[[u, b, w],[a, v]]+[[u, b, v],[a, w]]+a[b, u, v, w]+[a, u, v, w] b,
\end{gathered}
$$

e, portanto,

$$
\begin{gathered}
{[u, a][b, v, w]+[u, b][a, v, w]} \\
=[u, a b, v, w]-([u, a, w][b, v]+[u, a, v][b, w])-([u, b, w][a, v]+[u, b, v][a, w]) \\
+[[u, b],[a, v, w]]+[[u, b, w],[a, v]]+[[u, b, v],[a, w]] \\
-a[u, b, v, w]-[u, a, v, w] b .
\end{gathered}
$$


Por definição, $T^{(1)}=\mathbb{K}\langle X\rangle$ e $T^{(n)}, n \geq 2$, é o ideal bilateral de $\mathbb{K}\langle X\rangle$ gerado pelos comutadores $\left[a_{1}, \ldots, a_{n}\right]$ em que $a_{i} \in A$. Tendo em vista que o comutador $\left[a_{1}, \ldots, a_{n}\right]$ é linear em cada entrada, considere que esses $a_{i}$ 's são monômios de $\mathbb{K}\langle X\rangle$. Desta forma, os elementos de $T^{(n)}$ são combinações lineares de elementos da forma $b\left[a_{1}, \ldots, a_{n}\right] c$ em que $b, c \in \mathbb{K}\langle X\rangle$ e $a_{i}$ é um monômio de $\mathbb{K}\langle X\rangle$. Uma vez que

$$
b\left[a_{1}, \ldots, a_{n}\right] c=b c\left[a_{1}, \ldots, a_{n}\right]+b\left[\left[a_{1}, a_{2}\right], a_{3}, \ldots, a_{n}, c\right],
$$

então $T^{(n)}$ é gerado, como $\mathbb{K}$-módulo, pelos elementos da forma $b\left[a_{1}, \ldots, a_{n}\right]$ em que $b \in$ $\mathbb{K}\langle X\rangle$ e $a_{i}$ é um monômio de $\mathbb{K}\langle X\rangle$.

O lema abaixo é conhecido e pode, por exemplo, ser encontrado em [36].

Lema 3.6. Sejam $a_{1}, a_{2}, a_{3}, a_{4}, b_{1}, \ldots, b_{n-3} \in \mathbb{K}\langle X\rangle, n \geq 4$, e $\sigma \in S_{4}$. Então $\left[b_{1}, b_{2}, \ldots, b_{n-3}, a_{\sigma(1)}\right]\left[a_{\sigma(2)}, a_{\sigma(3)}, a_{\sigma(4)}\right] \equiv(-1)^{\sigma}\left[b_{1}, b_{2}, \ldots, b_{n-3}, a_{1}\right]\left[a_{2}, a_{3}, a_{4}\right] \quad\left(\bmod T^{(n)}\right)$. Demonstração. Fazendo as substituições $a=a_{1}, b=a_{4}, u=\left[b_{1}, \ldots, b_{n-3}\right]$ e $v=\left[a_{2}, a_{3}\right]$ na equação 3.1 , temos

$$
\left[b_{1}, \ldots, b_{n-3}, a_{1}\right]\left[a_{2}, a_{3}, a_{4}\right] \equiv-\left[b_{1}, \ldots, b_{n-3}, a_{4}\right]\left[a_{2}, a_{3}, a_{1}\right] \quad\left(\bmod T^{(n)}\right) \quad(\star) .
$$

Por outro lado, fazendo as substituições $a=a_{1}, b=a_{3}, u=\left[b_{1}, \ldots, b_{n-2}\right]$ e $v=a_{2}$ na equação 3.1, temos

$$
\left[b_{1}, \ldots, b_{n-2}, a_{1}\right]\left[a_{2}, a_{3}\right] \equiv-\left[b_{1}, \ldots, b_{n-2}, a_{3}\right]\left[a_{2}, a_{1}\right] \quad\left(\bmod T^{(n)}\right) \quad(\star \star) .
$$

Fazendo agora, na equação 3.2 , as substituições $a=a_{1}, b=a_{2}, v=a_{3}, w=a_{4}$ e $u=\left[b_{1}, \ldots, b_{n-3}\right]$, e usando $(\star \star)$ temos

$$
\left[b_{1}, \ldots, b_{n-3}, a_{1}\right]\left[a_{2}, a_{3}, a_{4}\right] \equiv-\left[b_{1}, \ldots, b_{n-3}, a_{2}\right]\left[a_{1}, a_{3}, a_{4}\right] \quad\left(\bmod T^{(n)}\right) \quad(\star \star \star) .
$$

O resultado segue de $(\star)$ e $(\star \star \star)$.

Seja $L^{(n)}, n>1$, o $\mathbb{K}$-módulo de $\mathbb{K}\langle X\rangle$ gerado pelo conjunto

$$
\left\{\left[a_{1}, \ldots, a_{n}\right] \mid a_{1}, \ldots, a_{n} \in \mathbb{K}\langle X\rangle\right\} .
$$

Além disso, admita que $L^{(1)}=\mathbb{K}\langle X\rangle$. 
Lema 3.7. Se $\frac{1}{3} \in \mathbb{K}$, então $L^{(n-2)} L^{(3)} \subset T^{(n)}$ para todo $n \geq 3$.

Demonstração. Claramente a inclusão ocorre quando $n=3$, basta ver que $L^{(1)} L^{(3)}=$ $T^{(3)}$. Se $n \geq 4$, então é suficiente mostrar que $\left[b_{1}, \ldots, b_{n-2}\right]\left[a_{1}, a_{2}, a_{3}\right] \in T^{(n)}$ para todos $b_{1}, \ldots, b_{n-2}, a_{1}, a_{2}, a_{3} \in \mathbb{K}\langle X\rangle$. Usando o lema 3.6 temos que

$$
\left[b_{1}, \ldots, b_{n-2}\right]\left[a_{2}, a_{3}, a_{1}\right] \equiv\left[b_{1}, \ldots, b_{n-2}\right]\left[a_{1}, a_{2}, a_{3}\right] \quad\left(\bmod T^{(n)}\right)
$$

$\mathrm{e}$

$$
\left[b_{1}, \ldots, b_{n-2}\right]\left[a_{3}, a_{1}, a_{2}\right] \equiv\left[b_{1}, \ldots, b_{n-2}\right]\left[a_{1}, a_{2}, a_{3}\right] \quad\left(\bmod T^{(n)}\right) .
$$

E uma vez que

$$
\left[b_{1}, \ldots, b_{n-2}\right]\left[a_{1}, a_{2}, a_{3}\right]+\left[b_{1}, \ldots, b_{n-2}\right]\left[a_{2}, a_{3}, a_{1}\right]+\left[b_{1}, \ldots, b_{n-2}\right]\left[a_{3}, a_{1}, a_{2}\right]=0,
$$

então $3\left[b_{1}, \ldots, b_{n-2}\right]\left[a_{1}, a_{2}, a_{3}\right] \equiv 0\left(\bmod T^{(n)}\right)$.

Note que na prova do lema 3.6 mostramos que $[a, c][b, d]+[a, d][b, c] \in T^{(n)}$ para todos $a \in L^{(n-2)}$ e $b, c, d \in \mathbb{K}\langle X\rangle$. Usaremos esse fato na demonstração do lema abaixo.

Lema 3.8. Se $\frac{1}{3} \in \mathbb{K}$, então $T^{(n)}=\mathbb{K}\langle X\rangle\left[T^{(n-2)}, \mathbb{K}\langle X\rangle, \mathbb{K}\langle X\rangle\right]$ para todo $n \geq 3$.

Demonstração. Para $n=3$ basta ver que $T^{(3)}=\mathbb{K}\langle X\rangle[\mathbb{K}\langle X\rangle, \mathbb{K}\langle X\rangle, \mathbb{K}\langle X\rangle]$. Suponha então que $n \geq 4$. Temos que $T^{(n)}$ é gerado como $\mathbb{K}$-módulo por elementos da forma $a\left[b_{1}, \ldots, b_{n}\right]$, em que $a, b_{1}, \ldots, b_{n} \in \mathbb{K}\langle X\rangle$. Portanto, $T^{(n)} \subset \mathbb{K}\langle X\rangle\left[T^{(n-2)}, \mathbb{K}\langle X\rangle, \mathbb{K}\langle X\rangle\right]$. Para mostrar que $\mathbb{K}\langle X\rangle\left[T^{(n-2)}, \mathbb{K}\langle X\rangle, \mathbb{K}\langle X\rangle\right] \subset T^{(n)}$ é suficiente verificar que $[b a, c, d] \in$ $T^{(n)}$ para todos $a \in L^{(n-2)}$ e $b, c, d \in \mathbb{K}\langle X\rangle$. Temos

$$
[a b, c, d]=a[b, c, d]+[a, c, d] b+[a, c][b, d]+[a, d][b, c]
$$

e uma vez que $[a, c, d] b \in T^{(n)}, a[b, c, d] \in L^{(n-2)} L^{(3)} \subset T^{(n)}$ e $[a, c][b, d]+[a, d][b, c] \in T^{(n)}$ segue o resultado.

Deve-se observar que a inclusão $\mathbb{K}\langle X\rangle\left[T^{(n-2)}, \mathbb{K}\langle X\rangle, \mathbb{K}\langle X\rangle\right] \subset T^{(n)}$, que é a parte não trivial do lema 3.8, é conhecida e aparece, por exemplo, em [22].

O teorema abaixo é o resultado principal desta seção e equivale ao teorema 1.7 enunciado no capítulo 1 (introdução). 
Teorema 3.9. Se $\frac{1}{3} \in \mathbb{K}$ e $F_{n-2}(n \geq 3)$ é um conjunto de polinômios de $\mathbb{K}\langle X\rangle$ que gera, como ideal bilateral, $T^{(n-2)}$, então $T^{(n)}$ é gerado, como ideal bilateral, pelos polinômios

(1) $\left[f, x_{i_{1}}, x_{i_{2}}\right]$;

(2) $f\left[x_{i_{1}}, x_{i_{2}}, x_{i_{3}}\right]$;

(3) $\left[f, x_{i_{1}}\right]\left[x_{i_{2}}, x_{i_{3}}, x_{i_{4}}\right]$;

(4) $\left[f, x_{i_{1}}\right]\left[x_{i_{2}}, x_{i_{3}}\right]+\left[f, x_{i_{2}}\right]\left[x_{i_{1}}, x_{i_{3}}\right]$;

(5) $f\left(\left[x_{i_{1}}, x_{i_{2}}\right]\left[x_{i_{3}}, x_{i_{4}}\right]+\left[x_{i_{1}}, x_{i_{3}}\right]\left[x_{i_{2}}, x_{i_{4}}\right]\right)$,

em que $f \in F_{n-2}$ e $x_{i_{j}} \in X$.

Demonstração. Segue do lema 3.8 que $T^{(n)}$ é gerado, como ideal bilateral, pelos elementos da forma $\left[u, a_{1}, a_{2}\right]$ onde $u \in T^{(n-2)}$ e $a_{1}, a_{2} \in \mathbb{K}\langle X\rangle$. Sendo $F_{n-2}(n \geq 3)$ um conjunto de polinômios de $\mathbb{K}\langle X\rangle$ que gera, como ideal bilateral, $T^{(n-2)}$, o resultado é uma consequência imediata do lema 3.5 (veja a conclusão da seção 3.1 deste capítulo).

Nos exemplos abaixo considere que $\frac{1}{3} \in \mathbb{K}$.

Exemplo 3.10. Seja $F_{2}=\left\{\left[x_{i}, x_{j}\right] \mid x_{i}, x_{j} \in X\right\}$. Conforme veremos abaixo (corolário 3.14), $T^{(2)}$ é gerado como ideal bilateral pelo conjunto $F_{2}$. Desta forma, pelo teorema 3.9, $T^{(4)}$ é gerado como ideal bilateral pelos polinômios

(11) $\left[x_{i_{1}}, x_{i_{2}}, x_{i_{3}}, x_{i_{4}}\right]$;

(12) $\left[x_{i_{1}}, x_{i_{2}}\right]\left[x_{i_{3}}, x_{i_{4}}, x_{i_{5}}\right]$;

(13) $\left[x_{i_{1}}, x_{i_{2}}, x_{i_{3}}\right]\left[x_{i_{4}}, x_{i_{5}}, x_{i_{6}}\right]$;

(14) $\left[x_{i_{1}}, x_{i_{2}}, x_{i_{3}}\right]\left[x_{i_{4}}, x_{i_{5}}\right]+\left[x_{i_{1}}, x_{i_{2}}, x_{i_{4}}\right]\left[x_{i_{3}}, x_{i_{5}}\right]$;

(15) $\left[x_{i_{1}}, x_{i_{2}}\right]\left(\left[x_{i_{3}}, x_{i_{4}}\right]\left[x_{i_{5}}, x_{i_{6}}\right]+\left[x_{i_{3}}, x_{i_{5}}\right]\left[x_{i_{4}}, x_{i_{6}}\right]\right)$,

em que $x_{i_{j}} \in X$. Note que

$$
\left[x_{i_{1}}, x_{i_{2}}, x_{i_{3}}\right]\left[x_{i_{4}}, x_{i_{5}}, x_{i_{6}}\right]=\left[x_{i_{1}}, x_{i_{2}}\right] x_{i_{3}}\left[x_{i_{4}}, x_{i_{5}}, x_{i_{6}}\right]-x_{i_{3}}\left[x_{i_{1}}, x_{i_{2}}\right]\left[x_{i_{4}}, x_{i_{5}}, x_{i_{6}}\right]
$$




$$
=\underbrace{\left[x_{i_{1}}, x_{i_{2}}\right]\left[x_{i_{4}}, x_{i_{5}}, x_{i_{6}}\right]}_{(12)} x_{i_{3}}-\left[x_{i_{1}}, x_{i_{2}}\right] \underbrace{\left[x_{i_{4}}, x_{i_{5}}, x_{i_{6}}, x_{i_{3}}\right]}_{(11)}-x_{i_{3}} \underbrace{\left[x_{i_{1}}, x_{i_{2}}\right]\left[x_{i_{4}}, x_{i_{5}}, x_{i_{6}}\right.}_{(12)}],
$$

e, portanto, os polinômios do tipo (13) estão no ideal bilateral gerado pelos polinômios dos tipos (11) e (12). Temos ainda

$$
\begin{aligned}
& {\left[x_{i_{1}}, x_{i_{2}}, x_{i_{3}}\right]\left[x_{i_{4}}, x_{i_{5}}\right]+\left[x_{i_{1}}, x_{i_{2}}, x_{i_{4}}\right]\left[x_{i_{3}}, x_{i_{5}}\right] } \\
= & \underbrace{\left[x_{i_{4}}, x_{i_{5}}\right]\left[x_{i_{1}}, x_{i_{2}}, x_{i_{3}}\right]}_{(12)}+\left[\left[x_{i_{1}}, x_{i_{2}}, x_{i_{3}}\right],\left[x_{i_{4}}, x_{i_{5}}\right]\right] \\
+ & \underbrace{\left[x_{i_{3}}, x_{i_{5}}\right]\left[x_{i_{1}}, x_{i_{2}}, x_{i_{4}}\right]}_{(12)}+\left[\left[x_{i_{1}}, x_{i_{2}}, x_{i_{4}}\right],\left[x_{i_{3}}, x_{i_{5}}\right]\right] .
\end{aligned}
$$

Mas, $\left[\left[x_{i_{1}}, x_{i_{2}}, x_{i_{3}}\right],\left[x_{i_{4}}, x_{i_{5}}\right]\right]$ e $\left[\left[x_{i_{1}}, x_{i_{2}}, x_{i_{4}}\right],\left[x_{i_{3}}, x_{i_{5}}\right]\right]$ estão no ideal bilateral gerado pelos polinômios do tipo (11), basta notar por exemplo que

$$
\begin{gathered}
{\left[\left[x_{i_{1}}, x_{i_{2}}, x_{i_{3}}\right],\left[x_{i_{4}}, x_{i_{5}}\right]\right]=\left[x_{i_{1}}, x_{i_{2}}, x_{i_{3}}, x_{i_{4}}, x_{i_{5}}\right]-\left[x_{i_{1}}, x_{i_{2}}, x_{i_{3}}, x_{i_{5}}, x_{i_{4}}\right]} \\
=\left[x_{i_{1}}, x_{i_{2}}, x_{i_{3}}, x_{i_{4}}\right] x_{i_{5}}-x_{i_{5}}\left[x_{i_{1}}, x_{i_{2}}, x_{i_{3}}, x_{i_{4}}\right]-\left[x_{i_{1}}, x_{i_{2}}, x_{i_{3}}, x_{i_{5}}\right] x_{i_{4}}+x_{i_{4}}\left[x_{i_{1}}, x_{i_{2}}, x_{i_{3}}, x_{i_{5}}\right] .
\end{gathered}
$$

Portanto, os polinômios do tipo (14) estão no ideal bilateral gerado pelos polinômios dos tipos (11) e (12). Assim, $T^{(4)}$ é gerado como ideal bilateral pelos polinômios dos tipos (11), (12) e (15).

Exemplo 3.11. Seja $F_{4}$ o conjunto formado pelos polinômios (11), (12) e (15). Desta forma, pelo teorema 3.9, $T^{(6)}$ é gerado como ideal bilateral pelos polinômios

(16) $\left[x_{i_{1}}, x_{i_{2}}, x_{i_{3}}, x_{i_{4}}, x_{i_{5}}, x_{i_{6}}\right]$;

(17) $\left[x_{i_{1}}, x_{i_{2}}, x_{i_{3}}, x_{i_{4}}\right]\left[x_{i_{5}}, x_{i_{6}}, x_{i_{7}}\right]$;

(18) $\left[x_{i_{1}}, x_{i_{2}}, x_{i_{3}}, x_{i_{4}}, x_{i_{5}}\right]\left[x_{i_{6}}, x_{i_{7}}, x_{i_{8}}\right]$;

(19) $\left[x_{i_{1}}, x_{i_{2}}, x_{i_{3}}, x_{i_{4}}, x_{i_{5}}\right]\left[x_{i_{6}}, x_{i_{7}}\right]+\left[x_{i_{1}}, x_{i_{2}}, x_{i_{3}}, x_{i_{4}}, x_{i_{6}}\right]\left[x_{i_{5}}, x_{i_{7}}\right]$;

(20) $\left[x_{i_{1}}, x_{i_{2}}, x_{i_{3}}, x_{i_{4}}\right]\left(\left[x_{i_{5}}, x_{i_{6}}\right]\left[x_{i_{7}}, x_{i_{8}}\right]+\left[x_{i_{5}}, x_{i_{7}}\right]\left[x_{i_{6}}, x_{i_{8}}\right]\right)$;

(21) $\left[\left[x_{i_{1}}, x_{i_{2}}\right]\left[x_{i_{3}}, x_{i_{4}}, x_{i_{5}}\right], x_{i_{6}}, x_{i_{7}}\right]$;

(22) $\left[x_{i_{1}}, x_{i_{2}}\right]\left[x_{i_{3}}, x_{i_{4}}, x_{i_{5}}\right]\left[x_{i_{6}}, x_{i_{7}}, x_{i_{8}}\right]$; 
(23) $\left[\left[x_{i_{1}}, x_{i_{2}}\right]\left[x_{i_{3}}, x_{i_{4}}, x_{i_{5}}\right], x_{i_{6}}\right]\left[x_{i_{7}}, x_{i_{8}}, x_{i_{9}}\right]$;

(24) $\left[\left[x_{i_{1}}, x_{i_{2}}\right]\left[x_{i_{3}}, x_{i_{4}}, x_{i_{5}}\right], x_{i_{6}}\right]\left[x_{i_{7}}, x_{i_{8}}\right]+\left[\left[x_{i_{1}}, x_{i_{2}}\right]\left[x_{i_{3}}, x_{i_{4}}, x_{i_{5}}\right], x_{i_{7}}\right]\left[x_{i_{6}}, x_{i_{8}}\right]$;

(25) $\left[x_{i_{1}}, x_{i_{2}}\right]\left[x_{i_{3}}, x_{i_{4}}, x_{i_{5}}\right]\left(\left[x_{i_{6}}, x_{i_{7}}\right]\left[x_{i_{8}}, x_{i_{9}}\right]+\left[x_{i_{6}}, x_{i_{8}}\right]\left[x_{i_{7}}, x_{i_{9}}\right]\right)$;

(26) $\left[\left[x_{i_{1}}, x_{i_{2}}\right]\left(\left[x_{i_{3}}, x_{i_{4}}\right]\left[x_{i_{5}}, x_{i_{6}}\right]+\left[x_{i_{3}}, x_{i_{5}}\right]\left[x_{i_{4}}, x_{i_{6}}\right]\right), x_{i_{7}}, x_{i_{8}}\right]$;

(27) $\left[x_{i_{1}}, x_{i_{2}}\right]\left(\left[x_{i_{3}}, x_{i_{4}}\right]\left[x_{i_{5}}, x_{i_{6}}\right]+\left[x_{i_{3}}, x_{i_{5}}\right]\left[x_{i_{4}}, x_{i_{6}}\right]\right)\left[x_{i_{7}}, x_{i_{8}}, x_{i_{9}}\right]$;

(28) $\left[\left[x_{i_{1}}, x_{i_{2}}\right]\left(\left[x_{i_{3}}, x_{i_{4}}\right]\left[x_{i_{5}}, x_{i_{6}}\right]+\left[x_{i_{3}}, x_{i_{5}}\right]\left[x_{i_{4}}, x_{i_{6}}\right]\right), x_{i_{7}}\right]\left[x_{i_{8}}, x_{i_{9}}, x_{i_{10}}\right]$;

(29) $\left[\left[x_{i_{1}}, x_{i_{2}}\right]\left(\left[x_{i_{3}}, x_{i_{4}}\right]\left[x_{i_{5}}, x_{i_{6}}\right]+\left[x_{i_{3}}, x_{i_{5}}\right]\left[x_{i_{4}}, x_{i_{6}}\right]\right), x_{i_{7}}\right]\left[x_{i_{8}}, x_{i_{9}}\right]$ $+\left[\left[x_{i_{1}}, x_{i_{2}}\right]\left(\left[x_{i_{3}}, x_{i_{4}}\right]\left[x_{i_{5}}, x_{i_{6}}\right]+\left[x_{i_{3}}, x_{i_{5}}\right]\left[x_{i_{4}}, x_{i_{6}}\right]\right), x_{i_{8}}\right]\left[x_{i_{7}}, x_{i_{9}}\right] ;$

(30) $\left[x_{i_{1}}, x_{i_{2}}\right]\left(\left[x_{i_{3}}, x_{i_{4}}\right]\left[x_{i_{5}}, x_{i_{6}}\right]+\left[x_{i_{3}}, x_{i_{5}}\right]\left[x_{i_{4}}, x_{i_{6}}\right]\right)\left(\left[x_{i_{7}}, x_{i_{8}}\right]\left[x_{i_{9}}, x_{i_{10}}\right]+\left[x_{i_{7}}, x_{i_{9}}\right]\left[x_{i_{8}}, x_{i_{10}}\right]\right)$, em que $x_{i_{j}} \in X$.

Exemplo 3.12. Temos que $T^{(1)}$ é gerado, como ideal bilateral, por 1 , isto é, $F_{1}=$ $\{1\}$. Desta forma, pelo teorema 3.9, $T^{(3)}$ é gerado como ideal bilateral pelos polinômios $\left[x_{i_{1}}, x_{i_{2}}, x_{i_{3}}\right] e\left[x_{i_{1}}, x_{i_{2}}\right]\left[x_{i_{3}}, x_{i_{4}}\right]+\left[x_{i_{1}}, x_{i_{3}}\right]\left[x_{i_{2}}, x_{i_{4}}\right]$

\subsection{Os comutadores que geram $T^{(n)}$ como um ideal bilateral}

Seja $M_{m}^{(n)}(m, n \geq 2)$ o ideal bilateral de $\mathbb{K}\langle X\rangle$ gerado pelos elementos da forma

$$
\left[a_{1}, \ldots, a_{n-1}, a_{n}\right]
$$

em que $a_{1}, \ldots, a_{n}$ são monômios de $\mathbb{K}\langle X\rangle$ tais que $a_{1}, \ldots, a_{n-1}$ têm grau menor ou igual a $m$ e $a_{n}$ tem grau 1 . Note que $M_{r}^{(n)} \subset M_{s}^{(n)} \subset T^{(n)}$ para todo $n \geq 2$ e para todos $2 \leq r<s$.

Lema 3.13. Os polinômios do tipo

$(*) \quad\left[a_{1}, \ldots, a_{n-1}, a_{n}\right] \quad(n \geq 2)$ 
em que $a_{1}, \ldots, a_{n}$ são monômios de $\mathbb{K}\langle X\rangle$ tais que $a_{1}, \ldots, a_{n-1}$ têm grau menor ou igual a $m$ estão em $M_{m}^{(n)}$.

Demonstração. A prova será feita por indução sobre o grau de $a_{n}$. Se $\operatorname{deg} a_{n}=1$, os polinômios do tipo $(*)$, por definição, pertencem a $M_{m}^{(n)}$. Suponha que $\operatorname{deg} a_{n}=r>1$ e que os polinômios do tipo $(*)$ tais que $\operatorname{deg} a_{n}<r$ estejam em $M_{m}^{(n)}$. Então $a_{n}=x_{i_{1}} \ldots x_{i_{r}}$, onde $x_{i_{j}} \in X$. Temos

$$
\begin{gathered}
{\left[a_{1}, \ldots, a_{n-1}, x_{i_{1}} x_{i_{2}} \ldots x_{i_{r-1}} x_{i_{r}}\right]} \\
=x_{i_{1}} x_{i_{2}} \ldots x_{i_{r-1}}\left[a_{1}, \ldots, a_{n-1}, x_{i_{r}}\right]+\left[a_{1}, \ldots, a_{n-1}, x_{i_{1}} x_{i_{2}} \ldots x_{i_{r-1}}\right] x_{i_{r}},
\end{gathered}
$$

e uma vez que $\left[a_{1}, \ldots, a_{n-1}, x_{i_{r}}\right]$ e $\left[a_{1}, \ldots, a_{n-1}, x_{i_{1}} x_{i_{2}} \ldots x_{i_{r-1}}\right]$ estão em $M_{m}^{(n)}$ pela hipótese de indução, então $\left[a_{1}, \ldots, a_{n}\right] \in M_{m}^{(n)}$.

Corolário 3.14. $T^{(2)}$ é gerado como ideal bilateral pelos elementos da forma $\left[x_{i}, x_{j}\right]$ para todos $x_{i}, x_{j} \in X$.

Demonstração. Por definição, $T^{(2)}$ é gerado, como ideal bilateral em $\mathbb{K}\langle X\rangle$, pelos elementos da forma $[a, b]$ em que $a, b \in \mathbb{K}\langle X\rangle$. Pela anti-simetria, segue que o ideal bilateral gerado pelos elementos da forma $\left[a, x_{j}\right]$ coincide com o ideal bilateral gerado pelos elementos da forma $\left[x_{j}, a\right]$. Assim, é suficiente observar que de acordo com a demonstração do lema acima, $[a, b]$ está no ideal gerado pelos elementos da forma $\left[a, x_{j}\right]$.

Uma consequência do corolário acima é que $M_{m}^{(2)}=T^{(2)}$ para todo $m \geq 2$. Nesta seção mostraremos que para todo $n \geq 2, M_{2}^{(n)}=T^{(n)}$ (quando $\frac{1}{6} \in \mathbb{K}$ ) e $M_{3}^{(n)}=T^{(n)}$ (quando $\frac{1}{3} \in \mathbb{K}$ ). Ambos os resultados aparecem aqui como consequência do teorema 3.9. No caminho para se chegar a esses resultados apresentaremos, em um conjunto de lemas, alguns elementos de $\mathbb{K}\langle X\rangle$ que estão em $M_{2}^{(n)}$ (e, portanto em $M_{3}^{(n)}$ ). Também será necessário obtermos elementos específicos de $M_{3}^{(n)}$. Isso será feito promovendo pequenas variações nas demonstrações de alguns lemas. Na sequência desta seção, as observações têm essa finalidade. Assim, os lemas dizem respeito a $M_{2}^{(n)}$ e as observações a $M_{3}^{(n)}$.

Na demonstração dos próximos três lemas usaremos a equação 3.1 dada anteriormente, isto é,

$$
[u, a][v, b]+[u, b][v, a]=-[u, a b, v]+[[u, b],[v, a]]+[u, a, v] b+a[u, b, v]
$$


em que $a, b, u, v \in \mathbb{K}\langle X\rangle$.

Lema 3.15. Seja $n \geq 3$ e sejam $a_{1}, \ldots, a_{n-2}$ monômios de $\mathbb{K}\langle X\rangle$ de grau menor ou igual a 2. Então

$$
\left[a_{1}, \ldots, a_{n-2}, x_{i}\right]\left[x_{k}, x_{l}, x_{j}\right]+\left[a_{1}, \ldots, a_{n-2}, x_{j}\right]\left[x_{k}, x_{l}, x_{i}\right] \equiv 0 \quad\left(\bmod M_{2}^{(n)}\right)
$$

Demonstração. Na equação 3.1 faça $a=x_{i}, b=x_{j}, u=\left[a_{1}, \ldots, a_{n-2}\right]$ e $v=\left[x_{k}, x_{l}\right]$. Desta forma, o primeiro membro dessa equação é a expressão que queremos mostrar que está em $M_{2}^{(n)}$. Quanto aos termos que estão no segundo membro da equação, temos

$$
\begin{gathered}
{[u, a b, v]=\left[a_{1}, \ldots, a_{n-2}, x_{i} x_{j},\left[x_{k}, x_{l}\right]\right],} \\
{[[u, b],[v, a]]=\left[a_{1}, \ldots, a_{n-2}, x_{j},\left[x_{k}, x_{l}, x_{i}\right]\right],} \\
{[u, a, v] b=\left[a_{1}, \ldots, a_{n-2}, x_{i},\left[x_{k}, x_{l}\right]\right] x_{j},} \\
a[u, b, v]=x_{i}\left[a_{1}, \ldots, a_{n-2}, x_{j},\left[x_{k}, x_{l}\right]\right] .
\end{gathered}
$$

Portanto, como consequência do lema 3.13, $[u, a b, v],[[u, b],[v, a]],[u, a, v] b, a[u, b, v] \in$ $M_{2}^{(n)}$, o que conclui a demonstração.

Na demonstração do lema a seguir usamos a relação

$$
[u,[a, b]]=[u, a, b]-[u, b, a]
$$

onde $u, a, b \in \mathbb{K}\langle X\rangle$.

Lema 3.16. Seja $n \geq 4$ e sejam $a_{1}, \ldots, a_{n-3}, c$ monômios de $\mathbb{K}\langle X\rangle$ de grau menor ou igual a 2. Então

$$
\left[a_{1}, \ldots, a_{n-3}, x_{i}\right]\left[c, x_{k}, x_{j}\right]+\left[a_{1}, \ldots, a_{n-3}, x_{j}\right]\left[c, x_{k}, x_{i}\right] \equiv 0 \quad\left(\bmod M_{2}^{(n)}\right) .
$$

Demonstração. Na equação 3.1 faça $a=x_{i}, b=x_{j}, u=\left[a_{1}, \ldots, a_{n-3}\right]$ e $v=\left[c, x_{k}\right]$. Desta forma, o primeiro membro dessa equação é a expressão que queremos mostrar que está em $M_{2}^{(n)}$. Quanto aos termos que estão no segundo membro da equação, temos

$$
\begin{gathered}
{[u, a b, v]=\left[a_{1}, \ldots, a_{n-3}, x_{i} x_{j},\left[c, x_{k}\right]\right]} \\
=\left[a_{1}, \ldots, a_{n-3}, x_{i} x_{j}, c, x_{k}\right]-\left[a_{1}, \ldots, a_{n-3}, x_{i} x_{j}, x_{k}, c\right],
\end{gathered}
$$




$$
\begin{gathered}
{[[u, b],[v, a]]=\left[a_{1}, \ldots, a_{n-3}, x_{j},\left[c, x_{k}, x_{i}\right]\right]} \\
=\left[a_{1}, \ldots, a_{n-3}, x_{j},\left[c, x_{k}\right], x_{i}\right]-\left[a_{1}, \ldots, a_{n-3}, x_{j}, x_{i},\left[c, x_{k}\right]\right] \\
=\left[\left[a_{1}, \ldots, a_{n-3}, x_{j}, c, x_{k}\right], x_{i}\right]-\left[\left[a_{1}, \ldots, a_{n-3}, x_{j}, x_{k}, c\right], x_{i}\right] \\
-\left[\left[a_{1}, \ldots, a_{n-3}, x_{j}, x_{i}, c\right], x_{k}\right]+\left[\left[a_{1}, \ldots, a_{n-3}, x_{j}, x_{i}, x_{k}\right], c\right] \\
{[u, a, v] b=\left[a_{1}, \ldots, a_{n-3}, x_{i},\left[c, x_{k}\right]\right] x_{j}} \\
=\left[a_{1}, \ldots, a_{n-3}, x_{i}, c, x_{k}\right] x_{j}-\left[a_{1}, \ldots, a_{n-3}, x_{i}, x_{k}, c\right] x_{j}
\end{gathered}
$$

e

$$
\begin{gathered}
a[u, b, v]=x_{i}\left[a_{1}, \ldots, a_{n-3}, x_{j},\left[c, x_{k}\right]\right] \\
=x_{i}\left[a_{1}, \ldots, a_{n-3}, x_{j}, c, x_{k}\right]-x_{i}\left[a_{1}, \ldots, a_{n-3}, x_{j}, x_{k}, c\right] .
\end{gathered}
$$

Portanto, como consequência do lema 3.13, $[u, a b, v],[[u, b],[v, a]],[u, a, v] b, a[u, b, v] \in$ $M_{2}^{(n)}$, o que conclui a demonstração.

Observação 3.17. Se na equação 3.1 fizermos $a=x_{i} x_{m}, b=x_{j}, u=\left[a_{1}, \ldots, a_{n-3}\right]\left(a_{i}\right.$ monômio de $\mathbb{K}\langle X\rangle$ de grau menor ou igual a 2), e $v=\left[x_{l}, x_{k}\right]$, então o primeiro membro dessa equação será igual a

$$
\left[a_{1}, \ldots, a_{n-3}, x_{i} x_{m}\right]\left[x_{l}, x_{k}, x_{j}\right]+\left[a_{1}, \ldots, a_{n-3}, x_{j}\right]\left[x_{l}, x_{k}, x_{i} x_{m}\right]
$$

Essa expressão é um elemento de $M_{2}^{(n)}$ ? Temos que o termo [u, ab, v], do segundo membro dessa equação, com as substituições acima ficará igual a

$$
\begin{gathered}
{\left[a_{1}, \ldots, a_{n-3}, x_{i} x_{m} x_{j},\left[x_{l}, x_{k}\right]\right]} \\
=\left[a_{1}, \ldots, a_{n-3}, x_{i} x_{m} x_{j}, x_{l}, x_{k}\right]-\left[a_{1}, \ldots, a_{n-3}, x_{i} x_{m} x_{j}, x_{k}, x_{l}\right] .
\end{gathered}
$$

E o que se pode afirmar, com certeza, é que esse termo está em $M_{3}^{(n)}$. Quanto aos demais termos do segundo membro da equação 3.1, as substituições resultarão em elementos de $M_{2}^{(n)}$ (refaça a demonstração do lema 3.16 com essas substituições). Desta forma,

$$
\left[a_{1}, \ldots, a_{n-3}, x_{i} x_{m}\right]\left[x_{l}, x_{k}, x_{j}\right]+\left[a_{1}, \ldots, a_{n-3}, x_{j}\right]\left[x_{l}, x_{k}, x_{i} x_{m}\right] \equiv 0 \quad\left(\bmod M_{3}^{(n)}\right)
$$


Lema 3.18. Seja $n \geq 3$ e sejam $a_{1}, \ldots, a_{n-2}$, c monômios de $\mathbb{K}\langle X\rangle$ de grau menor ou igual a 2. Então

$$
\left[a_{1}, \ldots, a_{n-2}, x_{i}\right]\left[c, x_{j}\right]+\left[a_{1}, \ldots, a_{n-2}, x_{j}\right]\left[c, x_{i}\right] \equiv 0 \quad\left(\bmod M_{2}^{(n)}\right) .
$$

Demonstração. Na equação 3.1 faça $a=x_{i}, b=x_{j}, u=\left[a_{1}, \ldots, a_{n-2}\right]$ e $v=c$. Desta forma, o primeiro membro dessa equação é a expressão que queremos mostrar que está em $M_{2}^{(n)}$. Quanto aos termos que estão no segundo membro da equação, temos

$$
\begin{gathered}
{[u, a b, v]=\left[a_{1}, \ldots, a_{n-2}, x_{i} x_{j}, c\right]} \\
{[[u, b],[v, a]]=\left[a_{1}, \ldots, a_{n-2}, x_{j},\left[c, x_{i}\right]\right],} \\
{[u, a, v] b=\left[a_{1}, \ldots, a_{n-2}, x_{i}, c\right] x_{j}}
\end{gathered}
$$

e

$$
a[u, b, v]=x_{i}\left[a_{1}, \ldots, a_{n-2}, x_{j}, c\right]
$$

Portanto, como consequência do lema 3.13, $[u, a b, v],[[u, b],[v, a]],[u, a, v] b, a[u, b, v] \in$ $M_{2}^{(n)}$, o que conclui a demonstração.

Observação 3.19. Se na equação 3.1 fizermos $a=x_{i} x_{l}, b=x_{j}, u=\left[a_{1}, \ldots, a_{n-2}\right] e$ $v=c\left(a_{1}, \ldots, a_{n}\right.$ e c monômios de $\mathbb{K}\langle X\rangle$ de grau menor ou igual a 2), então o primeiro membro dessa equação será igual a

$$
\left[a_{1}, \ldots, a_{n-2}, x_{i} x_{l}\right]\left[c, x_{j}\right]+\left[a_{1}, \ldots, a_{n-2}, x_{j}\right]\left[c, x_{i} x_{l}\right]
$$

Essa expressão é um elemento de $M_{2}^{(n)}$ ? Temos que o termo [u, ab, v], do segundo membro dessa equação, com as substituições acima ficará igual a

$$
\left[a_{1}, \ldots, a_{n-2}, x_{i} x_{l} x_{j}, c\right]
$$

E o que se pode afirmar, com certeza, é que esse termo está em $M_{3}^{(n)}$. Quanto aos demais termos do segundo membro da equação 3.1, as substituições resultarão em elementos de $M_{2}^{(n)}$ (refaça a demonstração do lema 3.18 com essas substituições). Desta forma,

$$
\left[a_{1}, \ldots, a_{n-2}, x_{i} x_{l}\right]\left[c, x_{j}\right]+\left[a_{1}, \ldots, a_{n-2}, x_{j}\right]\left[c, x_{i} x_{l}\right] \equiv 0 \quad\left(\bmod M_{3}^{(n)}\right)
$$


Na demonstração dos dois lemas seguintes usaremos a equação 3.2 dada anteriormente, isto é,

$$
\begin{gathered}
{[u, a][b, v, w]+[u, b][a, v, w]} \\
=[u, a b, v, w]-([u, a, w][b, v]+[u, a, v][b, w])-([u, b, w][a, v]+[u, b, v][a, w]) \\
+[[u, b],[a, v, w]]+[[u, b, w],[a, v]]+[[u, b, v],[a, w]]-a[u, b, v, w]-[u, a, v, w] b,
\end{gathered}
$$

em que $a, b, u, v, w \in \mathbb{K}\langle X\rangle$.

Lema 3.20. Seja $n \geq 3$ e sejam $a_{1}, \ldots, a_{n-2}$ monômios de $\mathbb{K}\langle X\rangle$ de grau menor ou igual a 2. Então

$$
\left[a_{1}, \ldots, a_{n-2}, x_{i}\right]\left[x_{j}, x_{k}, x_{l}\right]+\left[a_{1}, \ldots, a_{n-2}, x_{j}\right]\left[x_{i}, x_{k}, x_{l}\right] \equiv 0 \quad\left(\bmod M_{2}^{(n)}\right)
$$

Demonstração. Na equação 3.2 faça $a=x_{i}, b=x_{j}, u=\left[a_{1}, \ldots, a_{n-2}\right], v=x_{k}$ e $w=x_{l}$. Desta forma, o primeiro membro dessa equação é a expressão que queremos mostrar que está em $M_{2}^{(n)}$. Quanto aos termos que estão no segundo membro da equação, temos

$$
\begin{gathered}
{[u, a b, v, w]=\left[a_{1}, \ldots, a_{n-2}, x_{i} x_{j}, x_{k}, x_{l}\right]=\left[\left[a_{1}, \ldots, a_{n-2}, x_{i} x_{j}, x_{k}\right], x_{l}\right] \in M_{2}^{(n)},} \\
{[[u, b],[a, v, w]]=\left[a_{1}, \ldots, a_{n-2}, x_{j},\left[x_{i}, x_{k}, x_{l}\right]\right] \in M_{2}^{(n)} \quad(\text { pelo lema } 3.13),} \\
{[[u, b, w],[a, v]]=\left[\left[a_{1}, \ldots, a_{n-2}, x_{j}, x_{l}\right],\left[x_{i}, x_{k}\right]\right] \in M_{2}^{(n)},} \\
{[[u, b, v],[a, w]]=\left[\left[a_{1}, \ldots, a_{n-2}, x_{j}, x_{k}\right],\left[x_{i}, x_{l}\right]\right] \in M_{2}^{(n)},} \\
{[u, a, w][b, v]+[u, a, v][b, w]} \\
=\left[a_{1}, \ldots, a_{n-2}, x_{i}, x_{l}\right]\left[x_{j}, x_{k}\right]+\left[a_{1}, \ldots, a_{n-2}, x_{i}, x_{k}\right]\left[x_{j}, x_{l}\right] \in M_{2}^{(n)}, \\
{[u, b, w][a, v]+[u, b, v][a, w]} \\
=\left[a_{1}, \ldots, a_{n-2}, x_{j}, x_{l}\right]\left[x_{i}, x_{k}\right]+\left[a_{1}, \ldots, a_{n-2}, x_{j}, x_{k}\right]\left[x_{i}, x_{l}\right] \in M_{2}^{(n)}, \\
a[u, b, v, w]=x_{i}\left[\left[a_{1}, \ldots, a_{n-2}, x_{j}, x_{k}\right], x_{l}\right] \in M_{2}^{(n)}
\end{gathered}
$$

e

$$
[u, a, v, w] b=\left[\left[a_{1}, \ldots, a_{n-2}, x_{i}, x_{k}\right], x_{l}\right] x_{j} \in M_{2}^{(n)}
$$

de onde segue o resultado. 
Lema 3.21. Seja $n \geq 4$ e sejam $a_{1}, \ldots, a_{n-3}$ monômios de $\mathbb{K}\langle X\rangle$ de grau menor ou igual a 2. Então

$$
\left[a_{1}, \ldots, a_{n-3}, x_{i}\right]\left[x_{j}, x_{k}, x_{l}\right]+\left[a_{1}, \ldots, a_{n-3}, x_{j}\right]\left[x_{i}, x_{k}, x_{l}\right] \equiv 0 \quad\left(\bmod M_{2}^{(n)}\right)
$$

Demonstração. Na equação 3.2 faça $a=x_{i}, b=x_{j}, u=\left[a_{1}, \ldots, a_{n-3}\right], v=x_{k}$ e $w=x_{l}$. Desta forma, o primeiro membro dessa equação é a expressão que queremos mostrar que está em $M_{2}^{(n)}$. Quanto aos termos que estão no segundo membro da equação, temos

$$
\begin{gathered}
{[u, a b, v, w]=\left[a_{1}, \ldots, a_{n-3}, x_{i} x_{j}, x_{k}, x_{l}\right] \in M_{2}^{(n)},} \\
{[[u, b],[a, v, w]]=\left[a_{1}, \ldots, a_{n-3}, x_{j},\left[x_{i}, x_{k}, x_{l}\right]\right]} \\
=\left[a_{1}, \ldots, a_{n-3}, x_{j},\left[x_{i}, x_{k}\right], x_{l}\right]-\left[a_{1}, \ldots, a_{n-3}, x_{j}, x_{l},\left[x_{i}, x_{k}\right]\right] \\
=\left[a_{1}, \ldots, a_{n-3}, x_{j}, x_{i} x_{k}, x_{l}\right]-\left[a_{1}, \ldots, a_{n-3}, x_{j}, x_{k} x_{i}, x_{l}\right] \\
-\left[a_{1}, \ldots, a_{n-3}, x_{j}, x_{l},\left[x_{i}, x_{k}\right]\right] \in M_{2}^{(n)}(\text { pelo lema 3.13), }
\end{gathered}
$$$$
[[u, b, w],[a, v]]=\left[a_{1}, \ldots, a_{n-3}, x_{j}, x_{l},\left[x_{i}, x_{k}\right]\right] \in M_{2}^{(n)}(\text { pelo lema 3.13) }
$$$$
[[u, b, v],[a, w]]=\left[a_{1}, \ldots, a_{n-3}, x_{j}, x_{k},\left[x_{i}, x_{l}\right]\right] \in M_{2}^{(n)}(\text { pelo lema 3.13) }
$$

$$
[u, a, w][b, v]+[u, a, v][b, w]
$$

$=\left[a_{1}, \ldots, a_{n-3}, x_{i}, x_{l}\right]\left[x_{j}, x_{k}\right]+\left[a_{1}, \ldots, a_{n-3}, x_{i}, x_{k}\right]\left[x_{j}, x_{l}\right] \in M_{2}^{(n)}$ (pelo lema 3.18)

$$
[u, b, w][a, v]+[u, b, v][a, w]
$$

$=\left[a_{1}, \ldots, a_{n-3}, x_{j}, x_{l}\right]\left[x_{i}, x_{k}\right]+\left[a_{1}, \ldots, a_{n-3}, x_{j}, x_{k}\right]\left[x_{i}, x_{l}\right] \in M_{2}^{(n)}$ (pelo lema 3.18)

$$
a[u, b, v, w]=x_{i}\left[a_{1}, \ldots, a_{n-3}, x_{j}, x_{k}, x_{l}\right] \in M_{2}^{(n)}
$$

e

$$
[u, a, v, w] b=\left[a_{1}, \ldots, a_{n-3}, x_{i}, x_{k}, x_{l}\right] x_{j} \in M_{2}^{(n)}
$$

de onde segue o resultado. 
Observação 3.22. Se na equação 3.2 fizermos $a=x_{i}, b=x_{j} x_{m}, u=\left[a_{1}, \ldots, a_{n-3}\right]$ ( $a_{i}$ monômio de $\mathbb{K}\langle X\rangle$ de grau menor ou igual a 2), $v=x_{k}$ e $w=x_{l}$, então o primeiro membro dessa equação será igual a

$$
\left[a_{1}, \ldots, a_{n-3}, x_{i}\right]\left[x_{j} x_{m}, x_{k}, x_{l}\right]+\left[a_{1}, \ldots, a_{n-3}, x_{j} x_{m}\right]\left[x_{i}, x_{k}, x_{l}\right]
$$

Essa expressão é um elemento de $M_{2}^{(n)}$ ? Temos que o termo [u,ab,v,w], do segundo membro dessa equação, com as substituições acima ficará igual a

$$
\left[a_{1}, \ldots, a_{n-3}, x_{i} x_{j} x_{m}, x_{k}, x_{l}\right]
$$

E o que se pode afirmar, com certeza, é que esse termo está em $M_{3}^{(n)}$. Quanto aos demais termos do segundo membro da equação 3.2, as substituições resultarão em elementos de $M_{2}^{(n)}$ (refaça a demonstração do lema 3.21 com essas substituições). Desta forma,

$$
\left[a_{1}, \ldots, a_{n-3}, x_{i}\right]\left[x_{j} x_{m}, x_{k}, x_{l}\right]+\left[a_{1}, \ldots, a_{n-3}, x_{j} x_{m}\right]\left[x_{i}, x_{k}, x_{l}\right] \equiv 0 \quad\left(\bmod M_{3}^{(n)}\right) .
$$

Observação 3.23. Se na equação 3.2 fizermos $a=x_{i}, \quad b=x_{j}, u=\left[a_{1}, \ldots, a_{n-3}\right]\left(a_{i}\right.$ monômio de $\mathbb{K}\langle X\rangle$ de grau menor ou igual a 2), $v=x_{k}$ e $w=x_{l} x_{m}$, então o primeiro membro dessa equação será igual a

$$
\left[a_{1}, \ldots, a_{n-3}, x_{i}\right]\left[x_{j}, x_{k}, x_{l} x_{m}\right]+\left[a_{1}, \ldots, a_{n-3}, x_{j}\right]\left[x_{i}, x_{k}, x_{l} x_{m}\right]
$$

Essa expressão é um elemento de $M_{2}^{(n)}$ ? Temos que os termos $[u, a, w][b, v]+[u, a, v][b, w]$ $e[u, b, w][a, v]+[u, b, v][a, w]$, do segundo membro dessa equação, com as substituições acima ficarão iguais a

$$
\left[a_{1}, \ldots, a_{n-3}, x_{i}, x_{l} x_{m}\right]\left[x_{j}, x_{k}\right]+\left[a_{1}, \ldots, a_{n-3}, x_{i}, x_{k}\right]\left[x_{j}, x_{l} x_{m}\right]
$$

$e$

$$
\left[a_{1}, \ldots, a_{n-3}, x_{j}, x_{l} x_{m}\right]\left[x_{i}, x_{k}\right]+\left[a_{1}, \ldots, a_{n-3}, x_{j}, x_{k}\right]\left[x_{i}, x_{l} x_{m}\right]
$$

respectivamente. Segue da observação 3.19 que esses termos estão em $M_{3}^{(n)}$. Quanto aos demais termos do segundo membro da equação 3.2, as substituições resultarão em elementos de $M_{2}^{(n)}$ (refaça a demonstração do lema 3.21 com essas substituições). Desta forma,

$$
\left[a_{1}, \ldots, a_{n-3}, x_{i}\right]\left[x_{j}, x_{k}, x_{l} x_{m}\right]+\left[a_{1}, \ldots, a_{n-3}, x_{j}\right]\left[x_{i}, x_{k}, x_{l} x_{m}\right] \equiv 0 \quad\left(\bmod M_{3}^{(n)}\right) .
$$


Corolário 3.24. Seja $n \geq 3$ e sejam $a_{1}, \ldots, a_{n-2}$ monômios de $\mathbb{K}\langle X\rangle$ de grau menor ou igual a 2. Então

$$
\left[a_{1}, \ldots, a_{n-2}, x_{i_{\sigma(1)}}\right]\left[x_{i_{\sigma(2)}}, x_{i_{\sigma(3)}}, x_{i_{\sigma(4)}}\right] \equiv(-1)^{\sigma}\left[a_{1}, \ldots, a_{n-2}, x_{i_{1}}\right]\left[x_{i_{2}}, x_{i_{3}}, x_{i_{4}}\right] \quad\left(\bmod M_{2}^{(n)}\right)
$$

em que $\sigma \in S_{4}$.

Demonstração. É uma consequência dos lemas 3.15 e 3.20 .

Corolário 3.25. Seja $n \geq 4$ e sejam $a_{1}, \ldots, a_{n-3}$ monômios de $\mathbb{K}\langle X\rangle$ de grau menor ou igual a 2. Então

$$
\left[a_{1}, \ldots, a_{n-3}, x_{i_{\sigma(1)}}\right]\left[x_{i_{\sigma(2)}}, x_{i_{\sigma(3)}}, x_{i_{\sigma(4)}}\right] \equiv(-1)^{\sigma}\left[a_{1}, \ldots, a_{n-3}, x_{i_{1}}\right]\left[x_{i_{2}}, x_{i_{3}}, x_{i_{4}}\right] \quad\left(\bmod M_{2}^{(n)}\right)
$$

em que $\sigma \in S_{4}$.

Demonstração. É uma consequência dos lemas 3.16 e 3.21 .

Lema 3.26. Seja $n \geq 3$ e sejam $a_{1}, \ldots, a_{n-2}$ monômios de $\mathbb{K}\langle X\rangle$ de grau menor ou igual a 2. Se $\frac{1}{3} \in \mathbb{K}$, então

$$
\left[a_{1}, \ldots, a_{n-2}, x_{i}\right]\left[x_{j}, x_{k}, x_{l}\right] \equiv 0 \quad\left(\bmod M_{2}^{(n)}\right)
$$

Demonstração. Segue do corolário 3.24 que

$$
\left[a_{1}, \ldots, a_{n-2}, x_{i}\right]\left[x_{k}, x_{l}, x_{j}\right] \equiv\left[a_{1}, \ldots, a_{n-2}, x_{i}\right]\left[x_{j}, x_{k}, x_{l}\right] \quad\left(\bmod M_{2}^{(n)}\right)
$$

e

$$
\left[a_{1}, \ldots, a_{n-2}, x_{i}\right]\left[x_{l}, x_{j}, x_{k}\right] \equiv\left[a_{1}, \ldots, a_{n-2}, x_{i}\right]\left[x_{j}, x_{k}, x_{l}\right] \quad\left(\bmod M_{2}^{(n)}\right)
$$

Então

$$
\begin{gathered}
{\left[a_{1}, \ldots, a_{n-2}, x_{i}\right]\left[x_{j}, x_{k}, x_{l}\right]} \\
\equiv-\left[a_{1}, \ldots, a_{n-2}, x_{i}\right]\left[x_{k}, x_{l}, x_{j}\right]-\left[a_{1}, \ldots, a_{n-2}, x_{i}\right]\left[x_{l}, x_{j}, x_{k}\right] \\
\equiv-2\left[a_{1}, \ldots, a_{n-2}, x_{i}\right]\left[x_{j}, x_{k}, x_{l}\right] \quad\left(\bmod M_{2}^{(n)}\right)
\end{gathered}
$$

e, portanto

$$
3\left[a_{1}, \ldots, a_{n-2}, x_{i}\right]\left[x_{j}, x_{k}, x_{l}\right] \equiv 0 \quad\left(\bmod M_{2}^{(n)}\right) .
$$


Lema 3.27. Seja $n \geq 4$ e sejam $a_{1}, \ldots, a_{n-3}$ monômios de $\mathbb{K}\langle X\rangle$ de grau menor ou igual a 2. Se $\frac{1}{3} \in \mathbb{K}$, então

$$
\left[a_{1}, \ldots, a_{n-3}, x_{i}\right]\left[x_{j}, x_{k}, x_{l}\right] \equiv 0 \quad\left(\bmod M_{2}^{(n)}\right)
$$

Demonstração. É análoga ao do lema 3.26: usamos a identidade de Jacobi e o corolário 3.25 .

Lema 3.28. Seja $n \geq 4$ e sejam $a_{1}, \ldots, a_{n-3}$ monômios de $\mathbb{K}\langle X\rangle$ de grau menor ou igual a 2. Se $\frac{1}{6} \in \mathbb{K}$, então

$$
\left[a_{1}, \ldots, a_{n-3}, x_{i}\right]\left[x_{j} x_{k}, x_{l}, x_{m}\right] \equiv 0 \quad\left(\bmod M_{2}^{(n)}\right)
$$

Demonstração. Temos

$$
\begin{gathered}
{\left[a_{1}, \ldots, a_{n-3}, x_{i}\right]\left[x_{j}, x_{k}, x_{l} x_{m}\right]} \\
=\left[a_{1}, \ldots, a_{n-3}, x_{i}\right] x_{l}\left[x_{j}, x_{k}, x_{m}\right]+\left[a_{1}, \ldots, a_{n-3}, x_{i}\right]\left[x_{j}, x_{k}, x_{l}\right] x_{m} \\
=\left[a_{1}, \ldots, a_{n-3}, x_{i}, x_{l}\right]\left[x_{j}, x_{k}, x_{m}\right]+x_{l}\left(\left[a_{1}, \ldots, a_{n-3}, x_{i}\right]\left[x_{j}, x_{k}, x_{m}\right]\right) \\
+\left(\left[a_{1}, \ldots, a_{n-3}, x_{i}\right]\left[x_{j}, x_{k}, x_{l}\right]\right) x_{m},
\end{gathered}
$$

e uma vez que

$$
\begin{gathered}
{\left[a_{1}, \ldots, a_{n-3}, x_{i}, x_{l}\right]\left[x_{j}, x_{k}, x_{m}\right] \in M_{2}^{(n)} \quad(\text { pelo lema } 3.26)} \\
{\left[a_{1}, \ldots, a_{n-3}, x_{i}\right]\left[x_{j}, x_{k}, x_{m}\right] \in M_{2}^{(n)}(\text { pelo lema } 3.27)}
\end{gathered}
$$

e

$$
\left[a_{1}, \ldots, a_{n-3}, x_{i}\right]\left[x_{j}, x_{k}, x_{l}\right] \in M_{2}^{(n)} \quad \text { (pelo lema 3.27) }
$$

então

$$
\left[a_{1}, \ldots, a_{n-3}, x_{i}\right]\left[x_{j}, x_{k}, x_{l} x_{m}\right] \in M_{2}^{(n)}
$$

Mas

$$
\begin{gathered}
{\left[a_{1}, \ldots, a_{n-3}, x_{i}\right]\left[x_{j}, x_{k}, x_{l} x_{m}\right]} \\
=\left[a_{1}, \ldots, a_{n-3}, x_{i}\right]\left[x_{l} x_{m}, x_{k}, x_{j}\right]-\left[a_{1}, \ldots, a_{n-3}, x_{i}\right]\left[x_{l} x_{m}, x_{j}, x_{k}\right]
\end{gathered}
$$

e, portanto,

$$
\left[a_{1}, \ldots, a_{n-3}, x_{i}\right]\left[x_{l} x_{m}, x_{k}, x_{j}\right] \equiv\left[a_{1}, \ldots, a_{n-3}, x_{i}\right]\left[x_{l} x_{m}, x_{j}, x_{k}\right] \quad\left(\bmod M_{2}^{(n)}\right) .
$$


Usando esse resultado junto com o lema 3.16, temos

$$
\begin{gathered}
{\left[a_{1}, \ldots, a_{n-3}, x_{i}\right]\left[x_{j} x_{k}, x_{l}, x_{m}\right]} \\
\equiv-\left[a_{1}, \ldots, a_{n-3}, x_{m}\right]\left[x_{j} x_{k}, x_{l}, x_{i}\right] \equiv-\left[a_{1}, \ldots, a_{n-3}, x_{m}\right]\left[x_{j} x_{k}, x_{i}, x_{l}\right] \\
\equiv\left[a_{1}, \ldots, a_{n-3}, x_{l}\right]\left[x_{j} x_{k}, x_{i}, x_{m}\right] \equiv\left[a_{1}, \ldots, a_{n-3}, x_{l}\right]\left[x_{j} x_{k}, x_{m}, x_{i}\right] \\
\equiv-\left[a_{1}, \ldots, a_{n-3}, x_{i}\right]\left[x_{j} x_{k}, x_{m}, x_{l}\right] \equiv-\left[a_{1}, \ldots, a_{n-3}, x_{i}\right]\left[x_{j} x_{k}, x_{l}, x_{m}\right] \quad\left(\bmod M_{2}^{(n)}\right) .
\end{gathered}
$$

Logo, $2\left[a_{1}, \ldots, a_{n-3}, x_{i}\right]\left[x_{j} x_{k}, x_{l}, x_{m}\right] \equiv 0\left(\bmod M_{2}^{(n)}\right)$.

Observação 3.29. Segue dos lemas 3.27 e 3.28 que se $\frac{1}{6} \in \mathbb{K}$, então

$$
\left[a_{1}, \ldots, a_{n-3}, x_{i}\right]\left[c, x_{j}, x_{k}\right] \equiv 0 \quad\left(\bmod M_{2}^{(n)}\right)
$$

em que $a_{1}, \ldots, a_{n-3}$, c são monômios de $\mathbb{K}\langle X\rangle$ de grau menor ou igual a 2 .

Observação 3.30. Seja $n \geq 4$ e sejam $a_{1}, \ldots, a_{n-3}$ monômios de $\mathbb{K}\langle X\rangle$ de grau menor ou igual a 2. De acordo com a demonstração do lema 3.28, para que

$$
\left[a_{1}, \ldots, a_{n-3}, x_{i}\right]\left[x_{l} x_{m}, x_{k}, x_{j}\right] \equiv\left[a_{1}, \ldots, a_{n-3}, x_{i}\right]\left[x_{l} x_{m}, x_{j}, x_{k}\right] \quad\left(\bmod M_{2}^{(n)}\right)
$$

é suficiente que $\frac{1}{3} \in \mathbb{K}$.

Por outro lado, usando as observações 3.17, 3.22 e 3.23, temos

$$
\begin{gathered}
{\left[a_{1}, \ldots, a_{n-3}, x_{i}\right]\left[x_{l} x_{m}, x_{k}, x_{j}\right] \stackrel{(3.22)}{\equiv}-\left[a_{1}, \ldots, a_{n-3}, x_{l} x_{m}\right]\left[x_{i}, x_{k}, x_{j}\right]} \\
\stackrel{(3.17)}{\equiv}\left[a_{1}, \ldots, a_{n-3}, x_{j}\right]\left[x_{i}, x_{k}, x_{l} x_{m}\right] \equiv-\left[a_{1}, \ldots, a_{n-3}, x_{j}\right]\left[x_{k}, x_{i}, x_{l} x_{m}\right] \\
\stackrel{(3.23)}{\equiv}\left[a_{1}, \ldots, a_{n-3}, x_{k}\right]\left[x_{j}, x_{i}, x_{l} x_{m}\right] \stackrel{(3.17)}{\equiv}-\left[a_{1}, \ldots, a_{n-3}, x_{l} x_{m}\right]\left[x_{j}, x_{i}, x_{k}\right] \\
\equiv\left[a_{1}, \ldots, a_{n-3}, x_{l} x_{m}\right]\left[x_{i}, x_{j}, x_{k}\right] \stackrel{(3.22)}{\equiv}-\left[a_{1}, \ldots, a_{n-3}, x_{i}\right]\left[x_{l} x_{m}, x_{j}, x_{k}\right] \quad\left(\bmod M_{3}^{(n)}\right) .
\end{gathered}
$$

Desta forma, se $\frac{1}{3} \in \mathbb{K}$, então

$$
\left[a_{1}, \ldots, a_{n-3}, x_{i}\right]\left[x_{l} x_{m}, x_{k}, x_{j}\right] \equiv 0 \quad\left(\bmod M_{3}^{(n)}\right)
$$

Lema 3.31. Seja $n \geq 4$ e sejam $a_{1}, \ldots, a_{n-3}$ monômios de $\mathbb{K}\langle X\rangle$ de grau menor ou igual a 2. Se $\frac{1}{6} \in \mathbb{K}$, ent $\tilde{a} O$

$$
\left[a_{1}, \ldots, a_{n-3}, x_{i}\right]\left(\left[x_{j}, x_{k}\right]\left[x_{l}, x_{m}\right]+\left[x_{j}, x_{l}\right]\left[x_{k}, x_{m}\right]\right) \equiv 0 \quad\left(\bmod M_{2}^{(n)}\right) .
$$


Demonstração. Temos

$$
\begin{gathered}
{\left[a_{1}, \ldots, a_{n-3}, x_{i}\right]\left[x_{j} x_{m}, x_{k}, x_{l}\right]} \\
=\left[a_{1}, \ldots, a_{n-3}, x_{i}\right] x_{j}\left[x_{m}, x_{k}, x_{l}\right]+\left[a_{1}, \ldots, a_{n-3}, x_{i}\right]\left[x_{j}, x_{k}, x_{l}\right] x_{m} \\
-\left[a_{1}, \ldots, a_{n-3}, x_{i}\right]\left(\left[x_{j}, x_{k}\right]\left[x_{l}, x_{m}\right]+\left[x_{j}, x_{l}\right]\left[x_{k}, x_{m}\right]\right) \\
=\left[a_{1}, \ldots, a_{n-3}, x_{i}, x_{j}\right]\left[x_{m}, x_{k}, x_{l}\right]+x_{j}\left[a_{1}, \ldots, a_{n-3}, x_{i}\right]\left[x_{m}, x_{k}, x_{l}\right] \\
+\left[a_{1}, \ldots, a_{n-3}, x_{i}\right]\left[x_{j}, x_{k}, x_{l}\right] x_{m}-\left[a_{1}, \ldots, a_{n-3}, x_{i}\right]\left(\left[x_{j}, x_{k}\right]\left[x_{l}, x_{m}\right]+\left[x_{j}, x_{l}\right]\left[x_{k}, x_{m}\right]\right) .
\end{gathered}
$$

Portanto, o resultado é consequência do lema 3.26 e da observação 3.29.

Observação 3.32. Seja $n \geq 4$ e sejam $a_{1}, \ldots, a_{n-3}$ monômios de $\mathbb{K}\langle X\rangle$ de grau menor ou igual a 2. Na demonstração do lema 3.31, para verificar que

$$
\left[a_{1}, \ldots, a_{n-3}, x_{i}\right]\left(\left[x_{j}, x_{k}\right]\left[x_{l}, x_{m}\right]+\left[x_{j}, x_{l}\right]\left[x_{k}, x_{m}\right]\right)
$$

está em $M_{2}^{(n)}$, escrevemos esta expressão como combinação linear de outras 4 expressões, entre elas $\left[a_{1}, \ldots, a_{n-3}, x_{i}\right]\left[x_{j} x_{m}, x_{k}, x_{l}\right]$. Note que apenas nessa expressão é que utilizamos a hipótese de que $\frac{1}{6} \in \mathbb{K}$ para garantir que ela está em $M_{2}^{(n)}$. Nas demais, é suficiente que $\frac{1}{3} \in \mathbb{K}$ para que elas estejam em $M_{2}^{(n)}$ (ver lemas 3.26 e 3.27). Segue então da observação 3.30 que se $\frac{1}{3} \in \mathbb{K}$, então

$$
\left[a_{1}, \ldots, a_{n-3}, x_{i}\right]\left(\left[x_{j}, x_{k}\right]\left[x_{l}, x_{m}\right]+\left[x_{j}, x_{l}\right]\left[x_{k}, x_{m}\right]\right) \equiv 0 \quad\left(\bmod M_{3}^{(n)}\right)
$$

Conforme observamos anteriormente, $T^{(2)}=M_{m}^{(2)}$ para todo $m \geq 2$ (corolário 3.14). Além disso, com a hipótese de que $\frac{1}{3} \in \mathbb{K}$, temos que $T^{(3)}=M_{m}^{(3)}$ para todo $m \geq 2$. De fato, $T^{(3)}$ é gerado como ideal bilateral de $\mathbb{K}\langle X\rangle$ pelos elementos da forma
(i) $\left[x_{i_{1}}, x_{i_{2}}, x_{i_{3}}\right]$
(ii) $\left[x_{i_{1}}, x_{i_{2}}\right]\left[x_{i_{3}}, x_{i_{4}}\right]+\left[x_{i_{1}}, x_{i_{3}}\right]\left[x_{i_{2}}, x_{i_{4}}\right]$

(veja o exemplo 3.12). Claramente, os elementos da forma (i) estão em $M_{m}^{(3)}$ e, pelo lema 3.18 , os elementos da forma (ii) estão em $M_{2}^{(3)} \subset M_{m}^{(3)}$ para todo $m \geq 2$.

Lema 3.33. Se $\frac{1}{6} \in \mathbb{K}$, então $T^{(n)}=M_{2}^{(n)}(n \geq 2)$. 
Demonstração. Temos que $M_{2}^{(n)} \subset T^{(n)}$ para todo $n \geq 2$. A prova de que $T^{(n)} \subset M_{2}^{(n)}$ para todo $n \geq 2$ será feita por indução sobre $n$. A base da indução segue do comentário acima. Seja $n$ algum inteiro maior que 3 e suponha que $T^{(k)} \subset M_{2}^{(k)}$ para todo $k<n$. Desta forma, $T^{(n-2)}=M_{2}^{(n-2)}$. Uma vez que $M_{2}^{(n-2)}$ é gerado como ideal bilateral de $\mathbb{K}\langle X\rangle$ pelos elementos da forma $\left[a_{1}, a_{2}, \ldots, a_{n-3}, x_{i}\right]$ em que $a_{1}, a_{2}, \ldots, a_{n-3}$ são monômios de $\mathbb{K}\langle X\rangle$ de grau menor ou igual a 2 , segue do teorema 3.9 que os polinômios dos tipos

$$
\begin{aligned}
& {\left[\left[a_{1}, \ldots, a_{n-3}, x_{i}\right], x_{j}, x_{l}\right] ;} \\
& {\left[a_{1}, \ldots, a_{n-3}, x_{i}\right]\left[x_{j}, x_{k}, x_{l}\right] ;} \\
& {\left[\left[a_{1}, \ldots, a_{n-3}, x_{i}\right], x_{j}\right]\left[x_{k}, x_{l}, x_{m}\right] ;} \\
& {\left[\left[a_{1}, \ldots, a_{n-3}, x_{i}\right], x_{j}\right]\left[x_{k}, x_{l}\right]+\left[\left[a_{1}, \ldots, a_{n-3}, x_{i}\right], x_{k}\right]\left[x_{j}, x_{l}\right] ;} \\
& {\left[a_{1}, \ldots, a_{n-3}, x_{i}\right]\left(\left[x_{j}, x_{l}\right]\left[x_{m}, x_{k}\right]+\left[x_{j}, x_{m}\right]\left[x_{l}, x_{k}\right]\right) .}
\end{aligned}
$$

geram, como ideal bilateral, $T^{(n)}$. Então, basta mostrar que esses polinômios estão $M_{2}^{(n)}$. Os polinômios do tipo (36) claramente estão em $M_{2}^{(n)}$. Os polinômios dos tipos (37), (38) e (39) estão em $M_{2}^{(n)}$, respectivamente, pelos lemas 3.27, 3.26 e 3.18. Já os polinômios do tipo (40) estão em $M_{2}^{(n)}$ pelo lema 3.31 .

Se na demonstração do lema 3.33 substituirmos a hipótese de que $\frac{1}{6} \in \mathbb{K}$ por $\frac{1}{3} \in \mathbb{K}$, ainda assim os polinômios dos tipos (36)-(39) estarão em $M_{2}^{(n)}$. No entanto, não podemos garantir o mesmo para os polinômios do tipo (40). Mas, de acordo com a observação 3.32, com essa nova hipótese os polinômios dos tipos (40) estarão em $M_{3}^{(n)}$. Assim, temos uma segunda versão para o lema 3.33 .

Lema 3.34. Se $\frac{1}{3} \in \mathbb{K}$, então $T^{(n)}=M_{3}^{(n)}(n \geq 2)$..

\subsection{Resultado principal}

O próximo teorema é o resultado principal deste capítulo e equivale ao teorema 1.2 apresentado no capítulo 1 (introdução). 
Teorema 3.35. Seja $\mathbb{K}$ um anel associativo, unitário e comutativo. Seja A uma $\mathbb{K}$ álgebra unitária associativa e seja $\mathcal{E}=\left\{e_{1}, e_{2}, \ldots\right\}$ um conjunto gerador para $A$. Denote por $\mathcal{E}^{k}, k \geq 1$, o conjunto dos elementos de $A$ da forma $e_{i_{1}} e_{i_{2}} \ldots e_{i_{k}}$ em que $e_{i_{j}} \in \mathcal{E}$.

1) Se $\frac{1}{6} \in \mathbb{K}$, então $T^{(n)}(A)$ é gerado como ideal bilateral pelo conjunto

$$
\left\{\left[u_{1}, \ldots, u_{n}\right] \mid u_{i} \in \mathcal{E} \cup \mathcal{E}^{2}\right\}
$$

2) Se $\frac{1}{3} \in \mathbb{K}$, então $T^{(n)}(A)$ é gerado como ideal bilateral pelo conjunto

$$
\left\{\left[u_{1}, \ldots, u_{n}\right] \mid u_{i} \in \mathcal{E} \cup \mathcal{E}^{2} \cup \mathcal{E}^{3}\right\}
$$

Demonstração. Seja $X=\left\{x_{1}, x_{2}, \ldots\right\}$ e considere a aplicação $\varphi_{0}: X \longrightarrow \mathcal{E}$ definida por $\varphi_{0}\left(x_{i}\right)=e_{i}$ para todo $i=1,2, \ldots$ Seja $\varphi: \mathbb{K}\langle X\rangle \longrightarrow A$ o homomorfismo de álgebras associativas unitárias que extende $\varphi_{0}$, isto é, $\varphi\left(x_{i}\right)=\varphi_{0}\left(x_{i}\right)=e_{i}$ para todo $i=1,2, \ldots$ Claramente, esse homomorfismo é sobrejetivo. Denote por $R^{(n)}$ e por $S^{(n)}$, respectivamente, os ideais em $A$ gerados pelos conjuntos $\left\{\left[u_{1}, \ldots, u_{n}\right] \mid u_{i} \in \mathcal{E} \cup \mathcal{E}^{2}\right\}$ e $\left\{\left[u_{1}, \ldots, u_{n}\right] \mid u_{i} \in \mathcal{E} \cup \mathcal{E}^{2} \cup \mathcal{E}^{3}\right\}$. Segue dos lemas 3.33 e 3.34 que

1) $R^{(n)}=\varphi\left(M_{2}^{(n)}\right)=\varphi\left(T^{(n)}\right)=T^{(n)}(A)$ quando $\frac{1}{6} \in \mathbb{K}$.

2) $S^{(n)}=\varphi\left(M_{3}^{(n)}\right)=\varphi\left(T^{(n)}\right)=T^{(n)}(A)$ quando $\frac{1}{3} \in \mathbb{K}$.

Lembramos que uma $\mathbb{K}$-álgebra associativa unitária $B$ é dita Lie nilpotente se existir um inteiro positivo $n \geq 1$ tal que $\left[b_{1}, \ldots, b_{n+1}\right]=0$ para todo $b_{1}, \ldots, b_{n+1} \in B$. O menor inteiro $n$ com essa propriedade é a classe de Lie-nilpotência de B. Portanto, B é Lienilpotente de classe $\leq n$ se, e somente se, $T^{(n+1)}(B)=\{0\}$. Além disso, dizemos que $B$ é localmente Lie-nilpotente se toda subálgebra de $B$ finitamente gerada (como álgebra) é Lie-nilpotente. O corolário abaixo é uma consequência imediata do teorema 3.35 e corresponde ao corolário 1.3 dado no capítulo 1 (introdução).

Corolário 3.36. Seja A uma $\mathbb{K}$-álgebra associativa unitária e seja $\mathcal{E}=\left\{e_{1}, e_{2}, \ldots\right\}$ um conjunto gerador de A. Para que a $\mathbb{K}$-álgebra A seja Lie-nilpotente é suficiente que exista um inteiro $n \geq 1$ tal que 
- $\left[u_{1}, \ldots, u_{n+1}\right]=0$ para todos $u_{1}, \ldots, u_{n+1} \in \mathcal{E} \cup \mathcal{E}^{2}$ quando $\frac{1}{6} \in \mathbb{K} ;$ ou

- $\left[u_{1}, \ldots, u_{n+1}\right]=0$ para todos $u_{1}, \ldots, u_{n+1} \in \mathcal{E} \cup \mathcal{E}^{2} \cup \mathcal{E}^{3}$ quando $\frac{1}{3} \in \mathbb{K}$.

$\left(\mathcal{E}^{k}, k \geq 1\right.$, é o conjunto dos elementos de $A$ da forma $e_{i_{1}} e_{i_{2}} \ldots e_{i_{k}}$ em que $\left.e_{i_{j}} \in \mathcal{E}\right)$

Os resultados abaixo são obtidos tomando como hipótese o fato de que $\frac{1}{3} \in \mathbb{K}$. No entanto, como pode ser facilmente verificado, temos os mesmos resultados com a hipótese de que $\frac{1}{6} \in \mathbb{K}$.

Corolário 3.37. Suponha que $\frac{1}{3} \in \mathbb{K}$ e seja $B$ uma $\mathbb{K}$-álgebra associativa unitária finitamente gerada. Então $T^{(n)}(B)(n \geq 2)$ é finitamente gerado como ideal bilateral.

Demonstração. Seja $\mathcal{E}=\left\{e_{1}, \ldots, e_{r}\right\}$ um conjunto de geradores para $B$ e seja $\mathcal{E}^{k}(k \geq 1)$ o conjunto dos elementos de $B$ da forma $e_{i_{1}} \ldots e_{i_{k}}$ em que $e_{i_{j}} \in \mathcal{E}$. Segue do teorema 3.35 que $T^{(n)}(B)$ é gerado, como ideal bilateral, pelos elementos $\left[b_{1}, \ldots, b_{n}\right]$ em que $b_{1}, \ldots, b_{n} \in \mathcal{E} \cup$ $\mathcal{E}^{2} \cup \mathcal{E}^{3}$. Desta forma, existem $r+r^{2}+r^{3}$ possibilidades para cada $b_{i}, i=1, \ldots, n$. Portanto, o ideal bilateral $T^{(n)}(B)$ possui um conjunto gerador com $\left(r+r^{2}+r^{3}\right)^{n}$ elementos.

Corolário 3.38. Seja B uma $\mathbb{K}$-álgebra associativa unitária finitamente gerada tal que $\frac{1}{3} \in \mathbb{K}$. Suponha que cada subálgebra de Lie de $B^{(-)}$finitamente gerada seja nilpotente. Então $B$ é Lie-nilpotente.

Demonstração. Seja $\mathcal{E}=\left\{e_{1}, \ldots, e_{r}\right\}$ um conjunto de geradores para $B$ e seja $\mathcal{E}^{k}(k \geq 1)$ o conjunto dos elementos de $B$ da forma $e_{i_{1}} \ldots e_{i_{k}}$ em que $e_{i_{j}} \in \mathcal{E}$. Seja $G$ a subálgebra de Lie de $B^{(-)}$gerada por $\mathcal{E} \cup \mathcal{E}^{2} \cup \mathcal{E}^{3}$. Uma vez que o conjunto $\mathcal{E} \cup \mathcal{E}^{2} \cup \mathcal{E}^{3}$ tem $r+r^{2}+r^{3}$ elementos, então $G$ é finitamente gerada. Por hipótese, $G$ é nilpotente e, portanto, existe um inteiro $n \geq 2$ tal que $\left[g_{1}, \ldots, g_{n}\right]=0$ para todos $g_{1}, \ldots, g_{n} \in \mathcal{E} \cup \mathcal{E}^{2} \cup \mathcal{E}^{3}$. Assim, pelo corolário 3.36, $\left[b_{1}, \ldots, b_{n}\right]=0$ para todos $b_{1}, \ldots, b_{n} \in B$. Portanto, $B$ é Lie-nilpotente.

Vale lembrar que esses dois últimos corolários são equivalentes, respectivamente, aos corolários 1.4 e 1.5 dados no capítulo 1 (introdução). 


\section{Capítulo 4}

\section{O centro da álgebra de Lie associada a série central inferior de $\mathbb{Z}\langle X\rangle$}

Seja $\mathbb{K}$ um anel associativo, comutativo e unitário e seja $G$ uma $\mathbb{K}$-álgebra de Lie. Defina a série de ideais de Lie em $G$ indutivamente:

$$
\begin{gathered}
L^{(1)}(G)=G ; \\
L^{(2)}(G)=\left[L^{(1)}(G), G\right] ; \\
L^{(3)}(G)=\left[L^{(2)}(G), G\right] ; \\
\vdots \\
L^{(i+1)}(G)=\left[L^{(i)}(G), G\right] .
\end{gathered}
$$

Essa série é denominada de série central inferior de $G$. Temos que

$$
G=L^{(1)}(G) \supset L^{(2)}(G) \supset L^{(3)}(G) \supset \ldots
$$

Defina os sucessivos quocientes da série central por $B_{i}(G):=L^{(i)}(G) / L^{(i+1)}(G)$ e considere a soma direta

$$
B(G)=\bigoplus_{i \geq 1} B_{i}(G)
$$

Dados $a \in L^{(i)}(G)$ e $b \in L^{(j)}(G)$, defina em $B(G)$ o produto [, ] tal que

$$
\left[a+L^{(i+1)}(G), b+L^{(j+1)}(G)\right]:=[a, b]+L^{(i+j+1)}(G) .
$$


Assim , $\left[B_{i}(G), B_{j}(G)\right] \subset B_{i+j}(G)$. Temos que o $\mathbb{K}$-módulo $B(G)$ munido desse produto é uma álgebra de Lie graduada, denominada álgebra de Lie associada a série central inferior $(*)$. Além disso, $B(G)$ é gerada como álgebra de Lie por $B_{1}(G)$. Para maiores detalhes, veja a seção 2.6 do capítulo 2 .

Neste capítulo, considere que $G$ é a álgebra de Lie associada a álgebra associativa $\mathbb{Z}\langle X\rangle$, isto é, $G=\mathbb{Z}\langle X\rangle^{(-)}$e escreva $L^{(i)}(G)=L^{(i)}, B_{i}(G)=B_{i}$ e $B(G)=B$. Note que $L^{(i)}$ é o $\mathbb{Z}$-módulo gerado pelo conjunto

$$
\left\{\left[f_{1}, \ldots, f_{i}\right] \mid f_{1}, \ldots, f_{i} \in \mathbb{Z}\langle X\rangle\right\}
$$

Além disso, considere que

(1) $T^{(m)}$ é ideal bilateral em $\mathbb{Z}\langle X\rangle$ gerado como ideal pelos elementos $\left[f_{1}, \ldots, f_{m}\right]$ em que $f_{1}, \ldots, f_{m} \in \mathbb{Z}\langle X\rangle, m>1$. Note que $T^{(m)}$ é o $\mathbb{Z}$-módulo gerado pelos elementos $g_{1}\left[f_{1}, \ldots, f_{m}\right] g_{2}$ em que $f_{1}, \ldots, f_{m}, g_{1}, g_{2} \in \mathbb{Z}\langle X\rangle$;

(2) $P_{n}$ é o $\mathbb{Z}$-módulo dos polinômios multilineares nas variáveis $x_{1}, \ldots, x_{n}$;

(3) $T_{n}^{(m)}=T^{(m)} \cap P_{n}$;

(4) $L_{n}^{(m)}=L^{(m)} \cap P_{n}$;

(5) $H_{m_{1}, \ldots, m_{k}}$ é o $\mathbb{Z}$-módulo gerado pelos polinômios de $\mathbb{Z}\langle X\rangle$ nas variáveis $x_{1}, \ldots, x_{k}$ com multigrau $\left(m_{1}, \ldots, m_{k}\right)$.

Denote por $\mathcal{J}$ a imagem de $T^{(3)}$ em $B_{1}$ e defina o $\mathbb{Z}$-módulo $\bar{B}_{1}:=B_{1} / \mathcal{J}$. É conhecido que $\mathcal{J}$ está contido no centro de $B$ (veja, por exemplo, [17] e [8]). Desta forma,

$$
\bar{B}:=B / \mathcal{J}=\bar{B}_{1} \oplus \bigoplus_{i \geq 2} B_{i}
$$

é uma álgebra de Lie graduada.

Lembramos aqui a definição de isolador dada no capítulo 2. Seja $R$ um domínio e $M$ um $R$-módulo, definimos o isolador de um submódulo $N$ de $M$, denotado por $I(N)$, como o submódulo

$$
I(N)=\{a \in M \mid \exists r \in R, r \neq 0, \text { em que, } r a \in N\}
$$


Claramente, $N \subset I(N)$. É fácil ver que $M / N$ tem torção se, e somente se, o isolador de $N$ contém propriamente $N$ (isto é, $I(N)$ é maior que $N$ ). Bhupatiraju, P.I. Etingof et al em [8] mostraram que $\bar{B}_{1}$ tem muita torção e, portanto, o isolador de $\mathcal{J}$ é maior que $\mathcal{J}$. O resultado principal deste capítulo 4 consiste em mostrar que o isolador de $\mathcal{J}$ está contido no centro de $B$ (teorema 4.41).

\subsection{Uma base para o $\mathbb{Z}$-módulo $T_{n}^{(3)}$}

C. Bekh-Ochir e D. M. Riley ([7]) quando $\mathbb{K}$ é um corpo de característica zero apresentaram uma certa base linear para o espaço dos polinômios multilineares de grau $n$ em $\mathbb{K}\langle X\rangle$. Para tanto, eles usaram a bem conhecida base de Specht (veja [34]) desse espaço. Em seguida, eles mostraram que um subconjunto dessa base é uma base linear para o ideal em $\mathbb{K}\langle X\rangle$ gerado pelos comutadores $\left[a_{1}, a_{2}, a_{3}\right]$ em que $a_{1}, a_{2}$ e $a_{3}$ são polinômios multilineares de grau $n$. Nesta seção, mostramos que esses resultados continuam válidos em $\mathbb{Z}\langle X\rangle$.

Lembramos aqui a definição de produto regular de grau $n, n \geq 1$, dada no capítulo 1 .

(1) Sejam $i_{1}<\ldots<i_{k}$ inteiros tais $\left\{i_{1}, \ldots, i_{k}\right\} \subseteq\{1, \ldots, n\}$. Então o monômio $W\left(x_{i_{1}}, \ldots, x_{i_{k}}\right)=x_{i_{1}} \ldots x_{i_{k}}$ é denominado regular.

(2) Seja $\left\{j_{1}, \ldots, j_{l}\right\} \subseteq\{1, \ldots, n\}$ tal que $j_{1}$ é o elemento minimal desse conjunto. Então o comutador multilinear $\left[x_{j_{1}}, x_{j_{2}}, \ldots, x_{j_{l}}\right]$ (de grau $\geq 2$ ) é denominado regular.

(3) Um produto $C W \in P_{n}$, em que $C=C_{1} \ldots C_{s}$ (s $\left.\geq 0\right)$ é um produto de comutadores regulares e $W$ é um monômio regular, é também denominado regular se

(a) o grau de $C_{i}$ não cresce da esquerda para direita; e

(b) o índice da indeterminada inicial de comutadores $C_{i}$ de mesmo grau cresce da esquerda para direita.

De acordo com o que foi visto na seção 2.4 do capítulo 2, o conjunto de todos os produtos regulares de grau $n$ formam uma base para $P_{n}$, que denominamos de base de Specht para $P_{n}$. Denote por $\mathcal{A}_{n}$ o conjunto de todos os elementos da base de Specht de $P_{n}$ da forma $C_{1} \ldots C_{s} W$ em que deg $C_{1} \geq 3$. Claramente $\mathcal{A}_{n} \subset T_{n}^{(3)}$. 
Para todo inteiro $k \geq 1$, defina

$$
\begin{gathered}
S_{2 k}^{\prime}=:\left\{\sigma \in S_{2 k} \mid \sigma(1)<\sigma(2), \sigma(3)<\sigma(4), \ldots, \sigma(2 k-1)<\sigma(2 k)\right. \\
\text { e } \sigma(1)<\sigma(3)<\ldots<\sigma(2 k-1)\} .
\end{gathered}
$$

Note que $\sigma=1 \in S_{2 k}^{\prime}$. Para $k \geq 1, \sigma \in S_{2 k}^{\prime}$ e $i_{1}<\ldots<i_{2 k}$, defina

$$
u_{\sigma}\left(x_{i_{1}}, \ldots, x_{i_{2 k}}\right)=\left[x_{i_{\sigma(1)}}, x_{i_{\sigma(2)}}\right] \cdots\left[x_{i_{\sigma(2 k-1)}}, x_{i_{\sigma(2 k)}}\right] .
$$

Sejam

$$
\begin{gathered}
\mathcal{R}_{n}=\left\{u_{\sigma}\left(x_{i_{1}}, \ldots, x_{i_{2 k}}\right) x_{j_{1}} \ldots x_{j_{n-2 k}} \mid 4 \leq 2 k \leq n, 1 \neq \sigma \in S_{2 k}^{\prime},\right. \\
\left.i_{1}<\ldots<i_{2 k}, j_{1}<\ldots<j_{n-2 k} \text { e }\left\{i_{1}, \ldots, i_{2 k}, j_{1}, \ldots, j_{n-2 k}\right\}=\{1, \ldots, n\}\right\} \text { e } \\
\mathcal{R}_{n}^{\prime}=\left\{u_{1}\left(x_{i_{1}}, \ldots, x_{i_{2 k}}\right) x_{j_{1}} \ldots x_{j_{n-2 k}} \mid 0 \leq 2 k \leq n\right. \\
\left.i_{1}<\ldots<i_{2 k}, j_{1}<\ldots<j_{n-2 k}, \text { e }\left\{i_{1}, \ldots, i_{2 k}, j_{1}, \ldots, j_{n-2 k}\right\}=\{1, \ldots, n\}\right\} .
\end{gathered}
$$

Note que $\mathcal{A}_{n} \cup \mathcal{R}_{n} \cup \mathcal{R}_{n}^{\prime}$ é a base de Specht para $P_{n}$.

\section{Exemplo 4.1.}

$$
\begin{gathered}
\mathcal{A}_{4}=\left\{\left[x_{1}, x_{2}, x_{3}\right] x_{4},\left[x_{1}, x_{3}, x_{2}\right] x_{4},\left[x_{1}, x_{2}, x_{4}\right] x_{3},\left[x_{1}, x_{4}, x_{2}\right] x_{3},\right. \\
{\left[x_{1}, x_{3}, x_{4}\right] x_{2},\left[x_{1}, x_{4}, x_{3}\right] x_{2},\left[x_{2}, x_{3}, x_{4}\right] x_{1},} \\
{\left[x_{2}, x_{4}, x_{3}\right] x_{1},\left[x_{1}, x_{2}, x_{3}, x_{4}\right],\left[x_{1}, x_{2}, x_{4}, x_{3}\right],} \\
\left.\left[x_{1}, x_{3}, x_{2}, x_{4}\right],\left[x_{1}, x_{3}, x_{4}, x_{2}\right],\left[x_{1}, x_{4}, x_{2}, x_{3}\right],\left[x_{1}, x_{4}, x_{3}, x_{2}\right]\right\} ; \\
\mathcal{R}_{4}=\left\{\left[x_{1}, x_{3}\right]\left[x_{2}, x_{4}\right],\left[x_{1}, x_{4}\right]\left[x_{2}, x_{3}\right]\right\} \\
\mathcal{R}_{4}^{\prime}=\left\{x_{1} x_{2} x_{3} x_{4},\left[x_{1}, x_{2}\right] x_{3} x_{4},\left[x_{1}, x_{3}\right] x_{2} x_{4},\left[x_{1}, x_{4}\right] x_{2} x_{3},\right. \\
\left.\left[x_{2}, x_{3}\right] x_{1} x_{4},\left[x_{2}, x_{4}\right] x_{1} x_{3},\left[x_{3}, x_{4}\right] x_{1} x_{2},\left[x_{1}, x_{2}\right]\left[x_{3}, x_{4}\right]\right\} .
\end{gathered}
$$

Para cada $1 \neq \sigma \in S_{2 k}^{\prime}$ e $4 \leq 2 k \leq n$, defina

$$
z_{\sigma}\left(x_{1}, \ldots, x_{2 k}\right)=u_{1}\left(x_{1}, \ldots, x_{2 k}\right)-(-1)^{\sigma} u_{\sigma}\left(x_{1}, \ldots, x_{2 k}\right)
$$

Seja

$$
\begin{gathered}
\mathcal{B}_{n}=\left\{z_{\sigma}\left(x_{i_{1}}, \ldots, x_{i_{2 k}}\right) W\left(x_{j_{1}}, \ldots, x_{j_{n-2 k}}\right) \mid 4 \leq 2 k \leq n, 1 \neq \sigma \in S_{2 k}^{\prime},\right. \\
\left.i_{1}<\ldots<i_{2 k}, j_{1}<\ldots<j_{n-2 k} \text { e }\left\{i_{1}, \ldots, i_{2 k}, j_{1}, \ldots, j_{n-2 k}\right\}=\{1, \ldots, n\}\right\} .
\end{gathered}
$$


Exemplo 4.2. $\mathcal{B}_{4}=\left\{\left[x_{1}, x_{2}\right]\left[x_{3}, x_{4}\right]+\left[x_{1}, x_{3}\right]\left[x_{2}, x_{4}\right],\left[x_{1}, x_{2}\right]\left[x_{3}, x_{4}\right]-\left[x_{1}, x_{4}\right]\left[x_{2}, x_{3}\right]\right\}$.

Note que, pelo corolário $2.52, \mathcal{B}_{n} \subset T_{n}^{(3)}$.

Proposição 4.3. O conjunto $\mathcal{A}_{n} \cup \mathcal{B}_{n} \cup \mathcal{R}_{n}^{\prime}$ é uma base para $P_{n}$.

Demonstração. Dado $u_{\sigma} W \in \mathcal{R}_{n}$ temos que $u_{\sigma} W=(-1)^{\sigma} u_{1} W-(-1)^{\sigma} z_{\sigma} W$. Desta forma, $\mathcal{A}_{n} \cup \mathcal{B}_{n} \cup \mathcal{R}_{n}^{\prime}$ gera $P_{n}$. Seja

$$
\sum_{i} \alpha_{i} a_{i}+\sum_{j} \beta_{j} b_{j}+\sum_{k} \gamma_{k} c_{k}=0
$$

em que $a_{i} \in \mathcal{A}_{n}, b_{j} \in \mathcal{B}_{n}, c_{k} \in \mathcal{R}_{n}^{\prime}$ e $\alpha_{i}, \beta_{j}, \gamma_{k} \in \mathbb{Z}$. Para cada $j, b_{j}$ pode ser escrito como $b_{j}=d_{j} \pm e_{j}$ em que $d_{j} \in \mathcal{R}_{n}^{\prime}$ e $e_{j} \in \mathcal{R}_{n}$. Desta forma

$$
\sum_{i} \alpha_{i} a_{i} \pm \sum_{j} \beta_{j} e_{j}+\left(\sum_{k} \gamma_{k} c_{k}+\sum_{j} \beta_{j} d_{j}\right)=0
$$

Portanto, $\alpha_{i}=\beta_{j}=\gamma_{k}=0$, para todo $i, j$ e $k$.

Proposição 4.4. A imagem do conjunto $\mathcal{R}_{n}^{\prime}$ pelo homomorfismo canônico $P_{n} \longrightarrow P_{n} / T_{n}^{(3)}$ é uma base para o $\mathbb{Z}$-módulo quociente $P_{n} / T_{n}^{(3)}$.

Demonstração. Seja $f \in P_{n}$. Então, pela proposição 4.3

$$
f=\sum_{i} \alpha_{i} a_{i}+\sum_{j} \beta_{j} b_{j}+\sum_{k} \gamma_{k} c_{k}
$$

em que $a_{i} \in \mathcal{A}_{n}, b_{j} \in \mathcal{B}_{n}, c_{k} \in \mathcal{R}_{n}^{\prime}$ e $\alpha_{i}, \beta_{j}, \gamma_{k} \in \mathbb{Z}$. Logo $f+T_{n}^{(3)}=\sum_{k} \gamma_{k} c_{k}+T_{n}^{(3)}$. O resultado segue então da observação 2.58 .

Proposição 4.5. $\mathcal{A}_{n} \cup \mathcal{B}_{n}$ é uma base de $T_{n}^{(3)}$.

Demonstração. Como vimos, $\mathcal{A}_{n} \cup \mathcal{B}_{n} \subset T_{n}^{(3)}$. Seja $f \in T_{n}^{(3)} \subset P_{n}^{3}$. Então

$$
f=\sum_{i} \alpha_{i} a_{i}+\sum_{j} \beta_{j} b_{j}+\sum_{k} \gamma_{k} c_{k}
$$

em que $a_{i} \in \mathcal{A}_{n}, b_{j} \in \mathcal{B}_{n}, c_{k} \in \mathcal{R}_{n}^{\prime}$ e $\alpha_{i}, \beta_{j}, \gamma_{k} \in \mathbb{Z}$. Assim $\sum_{k} \gamma_{k} c_{k} \in T_{n}^{(3)}$. Mas, pela proposição $4.4, \gamma_{k}=0$ para todo inteiro $k$. Logo $\mathcal{A}_{n} \cup \mathcal{B}_{n}$ gera $T_{n}^{(3)}$ e, uma vez que esse conjunto está contido na base de $P_{n}$, é linearmente independente.

Denote por $V_{n}$ o $\mathbb{Z}$-módulo gerado pelo conjunto $\mathcal{R}_{n}^{\prime}$. Temos então, como consequência das proposições 4.3 e 4.5 , que $P_{n}=T_{n}^{(3)} \bigoplus V_{n}$. 


\subsection{O quociente $P_{n} / L_{n}^{(3)}$}

Nesta seção mostramos que dois dos resultados principais que aparecem em [7] para $\mathbb{K}\langle X\rangle$, quando $\mathbb{K}$ é um corpo de característica zero, são válidos em $\mathbb{Z}\langle X\rangle$. Um deles trata da decomposição de $\mathbb{Z}$-módulos $T_{n}^{(3)}=L_{n}^{(3)} \oplus T_{n-1}^{(3)} x_{n}$ e o outro diz respeito a uma base para o $\mathbb{Z}$-módulo $L_{n}^{(3)}$.

Nas demonstrações do lemas desta seção faremos uso de alguns resultados que estão elencados na afirmação abaixo. A demonstração é imediata.

Afirmação 4.6. Sejam $a, b, c, d \in \mathbb{Z}\langle X\rangle$. Então

1) $[a, b c]=b[a, c]+[a, b] c$;

2) $[a b, c, d]=a[b, c, d]+[a, c, d] b+[a, c][b, d]+[a, d][b, c]$;

3) $[a, b, c] d \equiv d[a, b, c]\left(\bmod L^{(3)}\right) e[a, b][c, d] \equiv[c, d][a, b]\left(\bmod L^{(3)}\right)$.

Lema 4.7. Sejam $a, b, c, d \in \mathbb{Z}\langle X\rangle$. Então

(i) $[a, b, c] d \equiv-[a, b, d] c\left(\bmod L^{(3)}\right)$;

(ii) $[a, b, c] d \equiv[c, d, a] b\left(\bmod L^{(3)}\right)$.

Demonstração.

(i) $[a, b, c] d \equiv[a, b, c] d-[a, b, c d] \equiv[a, b, c] d-(c[a, b, d]+[a, b, c] d) \equiv-[a, b, d] c\left(\bmod L^{(3)}\right)$.

(ii)

$$
\begin{gathered}
{[a, b, c] d \equiv[a, b, c] d-[a d, b, c] \equiv-[d, b, c] a-[a, b][d, c]-[a, c][d, b]} \\
\equiv[d, b, a] c-[d, c][a, b]-[a, c][d, b] \equiv-[b, d, a] c-[c, d][b, a]-[c, a][b, d] \\
\equiv-(c[b, d, a]+[c, d][b, a]+[c, a][b, d]+[c, d, a] b)+[c, d, a] b \\
\equiv-[c b, d, a]+[c, d, a] b \equiv[c, d, a] b \quad\left(\bmod L^{(3)}\right) .
\end{gathered}
$$

Para efeito de simplificação, ao longo desta seção, $a \equiv b$ significa $a \equiv b\left(\bmod L^{(3)}\right)$.

Lema 4.8. Sejam $a, b, c, d_{1}, \ldots, d_{k} \in \mathbb{Z}\langle X\rangle$ em que $k \geq 1$. Então

$$
\left[c, a, d_{1}, d_{2}, \ldots, d_{k}\right] b \equiv(-1)^{k+1}\left[d_{k}, b, d_{k-1}, d_{k-2}, \ldots, d_{2}, d_{1}, c\right] a
$$


Demonstração. A prova será feita por indução sobre $k$.

Para $k=1$, temos pelo lema 4.7 que $\left[c, a, d_{1}\right] b \equiv\left[d_{1}, b, c\right] a$. Suponha que o lema seja verdadeiro para algum $k \geq 1$. Assim

$$
\begin{gathered}
{\left[c, a, d_{1}, d_{2}, \ldots, d_{k}, d_{k+1}\right] b \equiv[[c, a], d_{1}, \underbrace{d_{2}, \ldots, d_{k+1}}_{k}] b} \\
\equiv(-1)^{k+1}\left[d_{k+1}, b, d_{k}, d_{k-1}, \ldots, d_{2},[c, a]\right] d_{1} \\
\text { Pela hipótese de indução }) \\
\left.\equiv(-1)^{k+1}\left(\left[d_{k+1}, b, d_{k}, d_{k-1}, \ldots, d_{2}, c, a\right] d_{1}-\left[\left[d_{k+1}, b, d_{k}, d_{k-1}, \ldots, d_{2}\right], a, c\right]\right] d_{1}\right) \\
\equiv(-1)^{k+1}\left(-\left[d_{k+1}, b, d_{k}, d_{k-1}, \ldots, d_{2}, c, d_{1}\right] a-\left[\left[c, d_{1}\right],\left[d_{k+1}, b, d_{k}, \ldots, d_{2}\right]\right] a\right) \\
(\text { Pelo lema } 4.7) \\
\equiv(-1)^{k+1}\left(-\left[d_{k+1}, b, d_{k}, d_{k-1}, \ldots, d_{2}, c, d_{1}\right]+\left[d_{k+1}, b, d_{k}, \ldots, d_{2},\left[c, d_{1}\right]\right]\right) a \\
\equiv(-1)^{k+1}\left(-\left[d_{k+1}, b, d_{k}, d_{k-1}, \ldots, d_{2}, d_{1}, c\right]\right) a \equiv(-1)^{k+2}\left[d_{k+1}, b, d_{k}, d_{k-1}, \ldots, d_{2}, d_{1}, c\right] a .
\end{gathered}
$$

O lema abaixo é bem conhecido e pode ser encontrado, por exemplo, em [17].

Lema 4.9. Sejam $a, b, c, d, e \in \mathbb{Z}\langle X\rangle$. Então $[[a, b, c] d, e] \equiv 0$.

Demonstração. Temos $[[a, b, c] d, e]=[a, b, c][d, e]+[a, b, c, e] d$.

Pelo lema 4.7, temos

$$
\begin{gathered}
{[a, b, c][d, e] \equiv-[a, b,[d, e]] c} \\
{[a, b, c, e] d \equiv[e, d,[a, b]] c \equiv[a, b,[d, e]] c .}
\end{gathered}
$$

Logo, $[[a, b, c] d, e] \equiv 0$.

Corolário 4.10. Sejam $u, v, w \in \mathbb{Z}\langle X\rangle$. Se $w \equiv 0$, então $[w v, u] \equiv 0$.

Demonstração. Temos que $w \in L^{(3)}$ e, portanto, $w=\sum_{i} \alpha_{i}\left[a_{i}, b_{i}, c_{i}\right]$, em que $\alpha_{i} \in \mathbb{Z}$ e $a_{i}, b_{i}, c_{i} \in \mathbb{Z}\langle X\rangle$. Dessa forma,

$$
[w v, u]=\left[\sum_{i} \alpha_{i}\left(\left[a_{i}, b_{i}, c_{i}\right] v\right), u\right]=\sum_{i} \alpha_{i}\left[\left[a_{i}, b_{i}, c_{i}\right] v, u\right] .
$$

O resultado segue então do lema 4.9 . 
Lema 4.11. Se $f \in \mathcal{A}_{n}$, então $f=f_{1}+f_{2}$, em que $f_{1} \in L_{n}^{(3)}$ e $f_{2} \in T_{n-1}^{(3)} x_{n}$.

Demonstração. Temos que $f$ é um elemento da base de Specht da forma $C_{1} C_{2} \ldots C_{s} W$ tal que $\operatorname{deg} C_{1} \geq 3$. Considere os seguintes casos:

$\left.1^{\mathrm{o}}\right) x_{n}$ está no comutador $C_{1}$.

Suponha que $C_{1}=\left[x_{i_{1}}, \ldots, x_{i_{t}}, x_{n}\right]$ em que $t \geq 2$. Pelo lema 4.7 , temos

$$
\left[x_{i_{1}}, \ldots, x_{i_{t}}, x_{n}\right] C_{2} \ldots C_{s} W \equiv-\left[x_{i_{1}}, \ldots, x_{i_{t}}, C_{2} \ldots C_{s} W\right] x_{n}
$$

Portanto, $f=f_{1}+f_{2}$, em que $f_{1} \in L_{n}^{(3)}$ e $f_{2} \in T_{n-1}^{(3)} x_{n}$.

Suponha que $C_{1}=\left[x_{i_{1}}, \ldots, x_{i_{t}}, x_{n}, x_{j_{1}}, \ldots, x_{j_{r}}\right]$ em que $t \geq 1$ e $r \geq 1$. Pelo lema 4.8 , temos

$$
\begin{gathered}
{\left[x_{i_{1}}, \ldots, x_{i_{t}}, x_{n}, x_{j_{1}}, \ldots, x_{j_{r}}\right] C_{2} \ldots C_{s} W} \\
\equiv(-1)^{r+1}\left[x_{j_{r}}, C_{2} \ldots C_{s} W, x_{j_{r-1}}, x_{j_{r-2}}, \ldots, x_{j_{2}}, x_{j_{1}},\left[x_{i_{1}}, \ldots, x_{i_{t}}\right]\right] x_{n} .
\end{gathered}
$$

Portanto, $f=f_{1}+f_{2}$, em que $f_{1} \in L_{n}^{(3)}$ e $f_{2} \in T_{n-1}^{(3)} x_{n}$. Note que se $f=C_{1}$, então $f \in L_{n}^{(3)}$.

$\left.2^{\circ}\right) x_{n}$ está em algum comutador $C_{l}$ de comprimento maior que 2 e com $l \neq 1$.

Suponha que $C_{l}=\left[x_{i_{1}}, \ldots, x_{i_{t}}, x_{n}, x_{j_{1}}, \ldots, x_{j_{r}}\right]$ em que $t \geq 1$ e $r \geq 0$, sendo que se $t=1$, então $r \geq 1$. Neste caso, escrevemos

$$
\begin{gathered}
f=C_{1} \ldots C_{l-1} C_{l} C_{l+1} \ldots C_{s} W \\
=C_{l} C_{1} \ldots C_{l-1} C_{l+1} \ldots C_{s} W-\left[C_{l}, C_{1} \ldots C_{l-1}\right] C_{l+1} \ldots C_{s} W \\
=\underbrace{\left[x_{i_{1}}, \ldots, x_{i_{t}}, x_{n}, x_{j_{1}}, \ldots, x_{j_{r}}\right] C_{1} \ldots C_{l-1} C_{l+1} \ldots C_{s} W}_{(1)} \\
-\underbrace{\left[x_{i_{1}}, \ldots, x_{i_{t}}, x_{n}, x_{j_{1}}, \ldots, x_{j_{r}}, C_{1} \ldots C_{l-1}\right] C_{l+1} \ldots C_{s} W}_{(2)} .
\end{gathered}
$$

Na expressão (1) aplicamos o caso anterior, enquanto que na expressão (2), usando o lema 4.8 , obtemos

$$
\begin{gathered}
{\left[x_{i_{1}}, \ldots, x_{i_{t}}, x_{n}, x_{j_{1}}, \ldots, x_{j_{r}}, C_{1} \ldots C_{l-1}\right] C_{l+1} \ldots C_{s} W} \\
\equiv(-1)^{r+2}\left[C_{1} \ldots C_{l-1}, C_{l+1} \ldots C_{s} W, x_{j_{r}}, x_{j_{r-1}}, x_{j_{r-2}}, \ldots, x_{j_{2}}, x_{j_{1}},\left[x_{i_{1}}, \ldots, x_{i_{t}}\right]\right] x_{n} .
\end{gathered}
$$


$\left.3^{\circ}\right) x_{n}$ está em algum comutador $C_{l}$ de comprimento 2 e com $l \neq 1$.

Assim

$$
\begin{gathered}
f=C_{1} \ldots C_{l-1}\left[x_{i}, x_{n}\right] C_{l+1} \ldots C_{s} W \\
=C_{1} \ldots C_{l-1} x_{i} x_{n} C_{l+1} \ldots C_{s} W-C_{1} \ldots C_{l-1} x_{n} x_{i} C_{l+1} \ldots C_{s} W \\
=\underbrace{\left(\left[C_{1} \ldots C_{l-1} x_{i} x_{n}, C_{l+1} \ldots C_{s} W\right]-\left[C_{1} \ldots C_{l-1} x_{n}, x_{i} C_{l+1} \ldots C_{s} W\right]\right)}_{f_{1}} \\
+\underbrace{\left(C_{l+1} \ldots C_{s} W C_{1} \ldots C_{l-1} x_{i}-x_{i} C_{l+1} \ldots C_{s} W C_{1} \ldots C_{l-1}\right) x_{n}}_{f_{2}} .
\end{gathered}
$$

Claramente $f_{2} \in T_{n-1}^{(3)} x_{n}$. Uma vez que $C_{1}$ tem comprimento maior que 3 , segue do lema 4.9 que $f_{1} \in L_{n}^{(3)}$.

O caso em que $x_{n}$ está em $W$ é trivial.

Lema 4.12. Sejam $a_{1}, \ldots, a_{2 k}, b \in \mathbb{Z}\langle X\rangle$, em que $k \geq 1$. Então

$$
\left[\left[a_{1}, a_{2}\right]\left[a_{3}, a_{4}\right] \ldots\left[a_{2 k-1}, a_{2 k}\right], b\right] \equiv 0 .
$$

Demonstração. A prova será feita por indução sobre $k$.

Para $k=1$, temos $\left[\left[a_{1}, a_{2}\right], b\right] \equiv 0$. Suponha que o lema seja verdadeiro para algum $k \geq 1$. Temos

$$
\begin{gathered}
0 \equiv\left[\left[\left[a_{1}, a_{2}\right] \ldots\left[a_{2 k-1}, a_{2 k}\right] a_{2 k+1}, a_{2 k+2}\right], b\right] \\
\equiv\left[\left[a_{1}, a_{2}\right] \ldots\left[a_{2 k-1}, a_{2 k}\right]\left[a_{2 k+1}, a_{2 k+2}\right], b\right]+\left[\left[\left[a_{1}, a_{2}\right] \ldots\left[a_{2 k-1}, a_{2 k}\right], a_{2 k+2}\right] a_{2 k+1}, b\right]
\end{gathered}
$$

Pela hipótese de indução temos $\left[\left[a_{1}, a_{2}\right] \ldots\left[a_{2 k-1}, a_{2 k}\right], a_{2 k+2}\right] \equiv 0$. Segue do corolário 4.10 que $\left[\left[\left[a_{1}, a_{2}\right] \ldots\left[a_{2 k-1}, a_{2 k}\right], a_{2 k+2}\right] a_{2 k+1}, b\right] \equiv 0$. Logo,

$$
\left[\left[a_{1}, a_{2}\right] \ldots\left[a_{2 k-1}, a_{2 k}\right]\left[a_{2 k+1}, a_{2 k+2}\right], b\right] \equiv 0 \text {. }
$$

Corolário 4.13. Sejam $a_{1}, \ldots, a_{2 k}, b, c \in \mathbb{Z}\langle X\rangle$, em que $k \geq 1$.

Se $u=\left[a_{1}, a_{2}\right]\left[a_{3}, a_{4}\right] \ldots\left[a_{2 k-1}, a_{2 k}\right]$, então

(i) $[u, b] c+[u, c] b \equiv 0$.

(ii) $[u b, c]+[u c, b] \equiv 0$.

(iii) $[b u c, a] \equiv[u b c, a]$. 
Demonstração.

(i) $0 \equiv[u, b c] \equiv[u, b] c+[u, c] b$.

(ii) $[u b, c]+[u c, b] \equiv[u, c] b+[b, c] u+[u, b] c+[c, b] u \equiv 0$.

(iii) Uma vez que $[u, b] \equiv 0$, então, pelo corolário $4.10,[[u, b] c, a] \equiv 0$.

Lema 4.14. Sejam $a_{1}, \ldots, a_{2 k}, b, c \in \mathbb{Z}\langle X\rangle$, em que $k \geq 1$. Então

$$
\left[\left[a_{1}, a_{2}\right]\left[a_{3}, a_{4}\right] \ldots\left[a_{2 k-1}, a_{2 k}\right] b, c\right] \equiv-\left[\left[b, a_{2}\right]\left[a_{3}, a_{4}\right] \ldots\left[a_{2 k-1}, a_{2 k}\right] a_{1}, c\right]
$$

Demonstração. Defina $u:=\left[a_{3}, a_{4}\right] \ldots\left[a_{2 k-1}, a_{2 k}\right]$. Temos

$$
\begin{gathered}
{\left[\left[b a_{1}, a_{2}\right] u, c\right]=\left[b\left[a_{1}, a_{2}\right] u, c\right]+\left[\left[b, a_{2}\right] a_{1} u, c\right]} \\
=\left[b,\left[a_{1}, a_{2}\right] u, c\right]+\left[\left[a_{1}, a_{2}\right] u b, c\right]+\left[\left[b, a_{2}\right] u a_{1}, c\right]+\left[\left[b, a_{2}\right]\left[a_{1}, u\right], c\right] .
\end{gathered}
$$

Segue então do lema 4.12 que $\left[\left[a_{1}, a_{2}\right] u b, c\right]+\left[\left[b, a_{2}\right] u a_{1}, c\right] \equiv 0$.

Corolário 4.15. Sejam $a_{1}, \ldots, a_{2 m}, b, c \in \mathbb{Z}\langle X\rangle$, em que $m \geq 1$. Então

$$
\left[\left[a_{1}, a_{2}\right] \ldots\left[a_{2 k-1}, a_{2 k}\right] \ldots\left[a_{2 m-1}, a_{2 m}\right] b, c\right] \equiv-\left[\left[a_{1}, a_{2}\right] \ldots\left[b, a_{2 k}\right] \ldots\left[a_{2 m-1}, a_{2 m}\right] a_{2 k-1}, c\right]
$$

Demonstração. Sejam $u=\left[a_{1}, a_{2}\right] \ldots\left[a_{2 k-3}, a_{2 k-2}\right] \quad$ e $v=\left[a_{2 k+1}, a_{2 k+2}\right] \ldots\left[a_{2 m-1}, a_{2 m}\right]$.

Segue do corolário 4.13-(iii) e do lema 4.14 que

$$
\left[u\left[a_{2 k-1}, a_{2 k}\right] v b, c\right] \equiv\left[\left[a_{2 k-1}, a_{2 k}\right] v u b, c\right] \equiv-\left[\left[b, a_{2 k}\right] v u a_{2 k-1}, c\right] \equiv-\left[u\left[b, a_{2 k}\right] v a_{2 k-1}, c\right] .
$$

Corolário 4.16. Sejam $a_{1}, \ldots, a_{2 k+1}, b \in \mathbb{Z}\langle X\rangle$, em que $k \geq 1$, e $\sigma \in S_{2 k+1}$. Então

$$
\left[\left[a_{\sigma(1)}, a_{\sigma(2)}\right] \ldots\left[a_{\sigma(2 k-1)}, a_{\sigma(2 k)}\right] a_{\sigma(2 k+1)}, b\right] \equiv(-1)^{\sigma}\left[\left[a_{1}, a_{2}\right] \ldots\left[a_{2 k-1}, a_{2 k}\right] a_{2 k+1}, b\right]
$$

Demonstração. É uma consequência imediata do corolário 4.15.

Observação 4.17. Considere as permutações $\theta, \sigma \in S_{2 n}$ tais que

$$
\theta=\left(\begin{array}{ccccc}
1 & 2 & \ldots & 2 n-1 & 2 n \\
2 & 3 & \ldots & 2 n & 1
\end{array}\right)
$$

e 


$$
\sigma=\left(\begin{array}{cccccccc}
1 & 2 & \ldots & 2 k-1 & 2 k & \ldots & 2 n-1 & 2 n \\
i_{1} & i_{2} & \ldots & i_{2 k-1} & i_{2 k} & \ldots & i_{2 n-1} & i_{2 n}
\end{array}\right)
$$

com $i_{2 k}=2 n$. Seja $\phi=\sigma \circ \underbrace{\theta \circ \ldots \circ \theta}_{2 k \text { vezes }}$. Então

$\phi=\left(\begin{array}{cccccccccc}1 & 2 & \ldots & 2 n-2 k-1 & 2 n-2 k & 2 n-2 k+1 & 2 n-2 k+2 & \ldots & 2 n-1 & 2 n \\ i_{2 k+1} & i_{2 k+2} & \ldots & i_{2 n-1} & i_{2 n} & i_{1} & i_{2} & \ldots & i_{2 k-1} & \underbrace{i_{2 k}}_{2 n}\end{array}\right)$

$e(-1)^{\phi}=(-1)^{\sigma} \cdot\left((-1)^{\theta}\right)^{2 k}=(-1)^{\sigma}$.

Seja $\pi$ uma permutação de $S_{2 n-1}$ definida por

$\pi=\left(\begin{array}{ccccccccc}1 & 2 & \ldots & 2 n-2 k-1 & 2 n-2 k & 2 n-2 k+1 & 2 n-2 k+2 & \ldots & 2 n-1 \\ i_{2 k+1} & i_{2 k+2} & \ldots & i_{2 n-1} & i_{2 n} & i_{1} & i_{2} & \ldots & i_{2 k-1}\end{array}\right)$.

Uma vez que $\phi(2 n)=2 n$, então

$$
(-1)^{\pi}=(-1)^{\phi}=(-1)^{\sigma} .
$$

Lema 4.18. Seja $m \geq 2$ e sejam $a_{1}, \ldots, a_{2 m} \in \mathbb{Z}\langle X\rangle$. Seja $\left\{i_{1}, i_{2}, \ldots, i_{2 m}\right\}$ uma permutação do conjunto $\{1,2, \ldots, 2 m\}$ tal que $\epsilon$ é o sinal dessa permutação. Além disso, suponha que $i_{2 k}=2 m$ para algum $0 \leq k \leq m$. Então

$$
\begin{gathered}
{\left[a_{1}, a_{2}\right] \ldots\left[a_{2 m-3}, a_{2 m-2}\right]\left[a_{2 m-1}, a_{2 m}\right]} \\
-\epsilon\left[a_{i_{1}}, a_{i_{2}}\right]\left[a_{i_{3}}, a_{i_{4}}\right] \ldots\left[a_{i_{2 k-3}}, a_{i_{2 k-2}}\right]\left[a_{i_{2 k-1}}, a_{i_{2 k}}\right]\left[a_{i_{2 k+1}}, a_{i_{2 k+2}}\right] \ldots\left[a_{i_{2 m-1}}, a_{i_{2 m}}\right] \\
\equiv\left(\left[\left[a_{1}, a_{2}\right] \ldots\left[a_{2 m-3}, a_{2 m-2}\right], a_{2 m-1}\right]\right. \\
\left.-\epsilon\left[\left[a_{i_{2 k+1}}, a_{i_{2 k+2}}\right] \ldots\left[a_{i_{2 m-1}}, a_{i_{2 m}}\right]\left[a_{i_{1}}, a_{i_{2}}\right] \ldots\left[a_{i_{2 k-3}}, a_{i_{2 k-2}}\right], a_{i_{2 k-1}}\right]\right) a_{2 m} .
\end{gathered}
$$

Demonstração. Seja $c=g-\epsilon f$, em que

$$
g=\left[\left[a_{1}, a_{2}\right] \ldots\left[a_{2 m-3}, a_{2 m-2}\right] a_{2 m-1}, a_{2 m}\right]
$$

$\mathrm{e}$

$$
f=\left[\left[a_{i_{2 k+1}}, a_{i_{2 k+2}}\right]\left[a_{i_{2 k+3}}, a_{i_{2 k+4}}\right] \ldots\left[a_{i_{2 m-1}}, a_{i_{2 m}}\right]\left[a_{i_{1}}, a_{i_{2}}\right] \ldots\left[a_{i_{2 k-3}}, a_{i_{2 k-2}}\right] a_{i_{2 k-1}}, a_{2 m}\right] .
$$


Segue do corolário 4.16 que $g \equiv(-1)^{\pi} f$, em que $\pi$ é a permutação de $S_{2 m-1}$ definida por $\pi=\left(\begin{array}{ccccccccc}1 & 2 & \ldots & 2 m-2 k-1 & 2 m-2 k & 2 m-2 k+1 & 2 m-2 k+2 & \ldots & 2 m-1 \\ i_{2 k+1} & i_{2 k+2} & \ldots & i_{2 m-1} & i_{2 m} & i_{1} & i_{2} & \ldots & i_{2 k-1}\end{array}\right)$.

De acordo com a observação $4.17,(-1)^{\pi}=\epsilon$ e, portanto,

$$
c \equiv 0
$$

Por outro lado,

$$
\begin{gathered}
c=\left[a_{1}, a_{2}\right] \ldots\left[a_{2 m-3}, a_{2 m-2}\right]\left[a_{2 m-1}, a_{2 m}\right]+\left[\left[a_{1}, a_{2}\right] \ldots\left[a_{2 m-3}, a_{2 m-2}\right], a_{2 m}\right] a_{2 m-1} \\
-\epsilon\left[a_{i_{2 k+1}}, a_{i_{2 k+2}}\right]\left[a_{i_{2 k+3}}, a_{i_{2 k+4}}\right] \ldots\left[a_{i_{2 m-1}}, a_{i_{2 m}}\right]\left[a_{i_{1}}, a_{i_{2}}\right] \ldots\left[a_{i_{2 k-3}}, a_{i_{2 k-2}}\right]\left[a_{i_{2 k-1}}, a_{2 m}\right] \\
-\epsilon\left[\left[a_{i_{2 k+1}}, a_{i_{2 k+2}}\right]\left[a_{i_{2 k+3}}, a_{i_{2 k+4}}\right] \ldots\left[a_{i_{2 m-1}}, a_{i_{2 m}}\right]\left[a_{i_{1}}, a_{i_{2}}\right] \ldots\left[a_{i_{2 k-3}}, a_{i_{2 k-2}}\right], a_{2 m}\right] a_{i_{2 k-1}} .
\end{gathered}
$$

Uma consequência do lema 4.12 é que

$$
\begin{aligned}
& {\left[a_{i_{2 k+1}}, a_{i_{2 k+2}}\right]\left[a_{i_{2 k+3}}, a_{i_{2 k+4}}\right] \ldots\left[a_{i_{2 m-1}}, a_{i_{2 m}}\right]\left[a_{i_{1}}, a_{i_{2}}\right] \ldots\left[a_{i_{2 k-3}}, a_{i_{2 k-2}}\right]\left[a_{i_{2 k-1}}, a_{2 m}\right]} \\
& \equiv\left[a_{i_{1}}, a_{i_{2}}\right]\left[a_{i_{3}}, a_{i_{4}}\right] \ldots\left[a_{i_{2 k-3}}, a_{i_{2 k-2}}\right]\left[a_{i_{2 k-1}}, a_{2 m}\right]\left[a_{i_{2 k+1}}, a_{i_{2 k+2}}\right] \ldots\left[a_{i_{2 m-1}}, a_{i_{2 m}}\right] .
\end{aligned}
$$

Além disso, segue do corolário 4.13-(i) que

$$
\left[\left[a_{1}, a_{2}\right] \ldots\left[a_{2 m-3}, a_{2 m-2}\right], a_{2 m}\right] a_{2 m-1} \equiv-\left[\left[a_{1}, a_{2}\right] \ldots\left[a_{2 m-3}, a_{2 m-2}\right], a_{2 m-1}\right] a_{2 m}
$$

e

$$
\begin{aligned}
& {\left[\left[a_{i_{2 k+1}}, a_{i_{2 k+2}}\right]\left[a_{i_{2 k+3}}, a_{i_{2 k+4}}\right] \ldots\left[a_{i_{2 m-1}}, a_{i_{2 m}}\right]\left[a_{i_{1}}, a_{i_{2}}\right] \ldots\left[a_{i_{2 k-3}}, a_{i_{2 k-2}}\right], a_{2 m}\right] a_{i_{2 k-1}} } \\
\equiv & -\left[\left[a_{i_{2 k+1}}, a_{i_{2 k+2}}\right]\left[a_{i_{2 k+3}}, a_{i_{2 k+4}}\right] \ldots\left[a_{i_{2 m-1}}, a_{i_{2 m}}\right]\left[a_{i_{1}}, a_{i_{2}}\right] \ldots\left[a_{i_{2 k-3}}, a_{i_{2 k-2}}\right], a_{2 k-1}\right] a_{2 m}
\end{aligned}
$$

Então

$$
\begin{gathered}
c \equiv\left[a_{1}, a_{2}\right] \ldots\left[a_{2 m-3}, a_{2 m-2}\right]\left[a_{2 m-1}, a_{2 m}\right] \\
-\epsilon\left[a_{i_{1}}, a_{i_{2}}\right]\left[a_{i_{3}}, a_{i_{4}}\right] \ldots\left[a_{i_{2 k-3}}, a_{i_{2 k-2}}\right]\left[a_{i_{2 k-1}}, a_{i_{2 k}}\right]\left[a_{i_{2 k+1}}, a_{i_{2 k+2}}\right] \ldots\left[a_{i_{2 m-1}}, a_{i_{2 m}}\right] \\
-\left(\left[\left[a_{1}, a_{2}\right] \ldots\left[a_{2 m-3}, a_{2 m-2}\right], a_{2 m-1}\right]\right. \\
\left.-\epsilon\left[\left[a_{i_{2 k+1}}, a_{i_{2 k+2}}\right] \ldots\left[a_{i_{2 m-1}}, a_{i_{2 m}}\right]\left[a_{i_{1}}, a_{i_{2}}\right] \ldots\left[a_{i_{2 k-3}}, a_{i_{2 k-2}}\right], a_{i_{2 k-1}}\right]\right) a_{2 m} .
\end{gathered}
$$

O resultado é uma consequência das equações 4.1 e 4.2 . 
Exemplo 4.19. Se $d=\left[x_{1}, x_{2}\right]\left[x_{3}, x_{4}\right]\left[x_{5}, x_{6}\right]\left[x_{7}, x_{8}\right]-\left[x_{1}, x_{8}\right]\left[x_{2}, x_{3}\right]\left[x_{4}, x_{7}\right]\left[x_{5}, x_{6}\right]$, ent $\tilde{a} o$

$$
d-\left(\left[\left[x_{1}, x_{2}\right]\left[x_{3}, x_{4}\right]\left[x_{5}, x_{6}\right], x_{7}\right]-\left[\left[x_{2}, x_{3}\right]\left[x_{4}, x_{7}\right]\left[x_{5}, x_{6}\right], x_{1}\right]\right) x_{8} \equiv 0 .
$$

Lema 4.20. Seja $m \geq 2$ e sejam $a_{1}, \ldots, a_{2 m}, b \in \mathbb{Z}\langle X\rangle$. Seja $\left\{i_{1}, i_{2}, \ldots, i_{2 m}\right\}$ uma permutação do conjunto $\{1,2, \ldots, 2 m\}$ tal que $\epsilon$ é o sinal dessa permutação. Se

$$
f=\left(\left[a_{1}, a_{2}\right] \ldots\left[a_{2 m-1}, a_{2 m}\right]-\epsilon\left[a_{i_{1}}, a_{i_{2}}\right] \ldots\left[a_{i_{2 m-1}}, a_{i_{2 m}}\right]\right) b
$$

então $f \equiv q a_{2 m}$, em que $q \in T^{(3)}$.

Demonstração. Suponha que $i_{2 k}=2 m$ para algum $0 \leq k \leq m$ e faça

$$
\begin{gathered}
c=\left[a_{1}, a_{2}\right] \ldots\left[a_{2 m-3}, a_{2 m-2}\right], \quad d=\left[a_{i_{1}}, a_{i_{2}}\right] \ldots\left[a_{i_{2 k-3}}, a_{i_{2 k-2}}\right] \\
\text { e } e=\left[a_{i_{2 k+1}}, a_{i_{2 k+2}}\right] \ldots\left[a_{i_{2 m-1}}, a_{i_{2 m}}\right] .
\end{gathered}
$$

Assim

$$
f=\left(c\left[a_{2 m-1}, a_{2 m}\right]-\epsilon d\left[a_{i_{2 k-1}}, a_{i_{2 k}}\right] e\right) b .
$$

Seja $g=c\left[a_{2 m-1}, a_{2 m} b\right]-\epsilon d\left[a_{i_{2 k-1}}, a_{2 m} b\right]$ e. Pelo lema 4.18

$$
g \equiv\left(\left[c, a_{2 m-1}\right]-\epsilon\left[e d, a_{i_{2 k-1}}\right]\right) a_{2 m} b
$$

Mas,

$$
\begin{gathered}
\left(\left[c, a_{2 m-1}\right]-\epsilon\left[e d, a_{i_{2 k-1}}\right]\right) a_{2 m} b \\
=b\left(\left[c, a_{2 m-1}\right]-\epsilon\left[e d, a_{i_{2 k-1}}\right]\right) a_{2 m}+\left[\left(\left[c, a_{2 m-1}\right]-\epsilon\left[e d, a_{i_{2 k-1}}\right]\right) a_{2 m}, b\right] .
\end{gathered}
$$

Segue do lema 4.12 e do corolário 4.10 que $\left[\left(\left[c, a_{2 m-1}\right]-\epsilon\left[e d, a_{i_{2 k-1}}\right]\right) a_{2 m}, b\right] \equiv 0$. Logo,

$$
g \equiv b\left(\left[c, a_{2 m-1}\right]-\epsilon\left[e d, a_{i_{2 k-1}}\right]\right) a_{2 m} .
$$

Por outro lado,

$$
\begin{gathered}
g=c a_{2 m}\left[a_{2 m-1}, b\right]+c\left[a_{2 m-1}, a_{2 m}\right] b-\epsilon d a_{2 m}\left[a_{i_{2 k-1}}, b\right] e-\epsilon d\left[a_{i_{2 k-1}}, a_{2 m}\right] b e \\
\equiv\left[a_{2 m-1}, b\right] c a_{2 m}+c\left[a_{2 m-1}, a_{2 m}\right] b-\epsilon\left[a_{i_{2 k-1}}, b\right] e d a_{2 m}-\epsilon d\left[a_{i_{2 k-1}}, a_{2 m}\right] e b-\epsilon d\left[a_{i_{2 k-1}}, a_{2 m}\right][b, e] .
\end{gathered}
$$

Assim

$$
g \equiv f+\left[a_{2 m-1}, b\right] c a_{2 m}-\epsilon\left[a_{i_{2 k-1}}, b\right] e d a_{2 m}-\epsilon d\left[a_{i_{2 k-1}}, a_{2 m}\right][b, e] .
$$


Segue então das equações 4.3 e 4.4 que

$$
f \equiv\left(b\left[c, a_{2 m-1}\right]-\epsilon b\left[e d, a_{i_{2 k-1}}\right]-\left[a_{2 m-1}, b\right] c+\epsilon\left[a_{i_{2 k-1}}, b\right] e d\right) a_{2 m}+\epsilon d\left[a_{i_{2 k-1}}, a_{2 m}\right][b, e] .
$$

Mas,

$$
\begin{gathered}
d\left[a_{i_{2 k-1}}, a_{2 m}\right][b, e] \equiv[b, e] d\left[a_{i_{2 k-1}}, a_{2 m}\right] \equiv[b, e] d a_{i_{2 k-1}} a_{2 m}-[b, e] d a_{2 m} a_{i_{2 k-1}} \\
\equiv[b, e] d a_{i_{2 k-1}} a_{2 m}-a_{i_{2 k-1}}[b, e] d a_{2 m}-\left[[b, e] d a_{2 m}, a_{i_{2 k-1}}\right] \\
\equiv\left[[b, e] d, a_{i_{2 k-1}}\right] a_{2 m}-\left[[b, e] d a_{2 m}, a_{i_{2 k-1}}\right]
\end{gathered}
$$

e uma vez que $[b, e] \equiv 0$, segue então do corolário 4.10 que $\left[[b, e] d a_{2 m}, a_{i_{2 k-1}}\right] \equiv 0$. Logo,

$$
f \equiv\left(b\left[c, a_{2 m-1}\right]-\epsilon b\left[e d, a_{i_{2 k-1}}\right]-\left[a_{2 m-1}, b\right] c+\epsilon\left[a_{i_{2 k-1}}, b\right] e d+\epsilon\left[[b, e] d, a_{i_{2 k-1}}\right]\right) a_{2 m} .
$$

Pelo lema 4.12 e pelo corolário 4.10 temos que

$$
\left[c, a_{2 m-1}\right],\left[e d, a_{i_{2 k-1}}\right],\left[[b, e] d, a_{i_{2 k-1}}\right] \in L^{(3)}
$$

e, portanto

$$
b\left[c, a_{2 m-1}\right]-\epsilon b\left[e d, a_{i_{2 k-1}}\right]+\epsilon\left[[b, e] d, a_{i_{2 k-1}}\right] \in T^{(3)} .
$$

Faça $h=\left[a_{2 m-1}, b\right] c-\epsilon\left[a_{i_{2 k-1}}, b\right] e d$. Então, pelo lema 4.12

$$
h \equiv c\left[a_{2 m-1}, b\right]-\epsilon d\left[a_{i_{2 k-1}}, b\right] e .
$$

Mas, pela proposição 2.52, $c\left[a_{2 m-1}, b\right]-\epsilon d\left[a_{i_{2 k-1}}, b\right] e \in T^{(3)}$. Portanto, $h \in T^{(3)}$. Segue então que $f \equiv q a_{2 m}$ em que $q \in T^{(3)}$.

Lema 4.21. Se $f \in \mathcal{B}_{n}$, então $f=f_{1}+f_{2}$, em que $f_{1} \in L_{n}^{(3)}$ e $f_{2} \in T_{n-1}^{(3)} x_{n}$

Demonstração. Conforme foi definido anteriormente, $\mathcal{B}_{n}, n \geq 4$, é o conjunto de todos os polinômios da forma

$$
\left(\left[x_{i_{1}}, x_{i_{2}}\right] \ldots\left[x_{i_{2 k-1}}, x_{i_{2 k}}\right]-(-1)^{\sigma}\left[x_{i_{\sigma(1)}}, x_{i_{\sigma(2)}}\right] \ldots\left[x_{i_{\sigma(2 k-1)}}, x_{i_{\sigma(2 k)}}\right]\right) x_{j_{1}} \ldots x_{j_{n-2 k}}
$$

em que

- $4 \leq 2 k \leq n$; 
- $1 \neq \sigma \in S_{n}$ é tal que $\sigma(1)<\sigma(2), \sigma(3)<\sigma(4), \ldots, \sigma(2 k-1)<\sigma(2 k)$ e $\sigma(1)<$ $\sigma(3)<\ldots<\sigma(2 k-1)$

- $i_{1}<\ldots<i_{2 k}, j_{1}<\ldots<j_{n-2 k} \quad \mathrm{e}$

- $\left\{i_{1}, \ldots, i_{2 k}, j_{1}, \ldots, j_{n-2 k}\right\}=\{1, \ldots, n\}$.

Note que $j_{n-2 k}=n$ ou $i_{2 k}=n$. Se $f \in \mathcal{B}_{n}$ é tal que $j_{n-2 k}=n$, então, pela proposição 2.52, $f=g x_{n}$, em que $g \in T_{n-1}^{(3)}$. Se $f \in \mathcal{B}_{n}$ é tal que $i_{2 k}=n$, então, pelo lema 4.20, $f=g+q x_{n}$, em que $g \in L_{n}^{(3)}$ e $q \in T_{n-1}^{(3)}$.

\section{Lema 4.22. O Conjunto}

$\mathcal{C}=\left\{\left[x_{n}, x_{\sigma(1)} \ldots x_{\sigma(n-1)}\right],\left[x_{n} x_{\sigma(1)} \ldots x_{\sigma(k)}, x_{\sigma(k+1)} \ldots x_{\sigma(n-1)}\right] \mid \sigma \in S_{n-1}, k \in\{1, \ldots, n-2\}\right\}$ é uma base para $L_{n}^{(2)}$.

Demonstração. De fato, note que se $f \in L_{n}^{(2)}$ então $f$ é uma combinação linear de elementos da forma $\left[x_{i_{1}} \ldots x_{i_{k}}, x_{i_{k+1}} \ldots x_{i_{n}}\right]$ em que $\left\{i_{1}, \ldots, i_{n}\right\}=\{1, \ldots, n\}$. Podemos supor que $x_{n}$ está na $1^{\circ}$ parte desses comutadores e usando a relação $\left[u x_{n} v, w\right]=\left[x_{n} v, w u\right]-\left[x_{n} v w, u\right]$ segue que os elementos de $\mathcal{C}$ geram $L_{n}^{(2)}$.

Seja $f \in \operatorname{span}(\mathcal{C})$. Então

$$
f=\sum_{\sigma \in S_{n-1}}\left(\alpha_{\sigma}^{(0)}\left[x_{n}, x_{\sigma(1)} \ldots x_{\sigma(n-1)}\right]+\sum_{k=1}^{n-2} \alpha_{\sigma}^{(k)}\left[x_{n} x_{\sigma(1)} \ldots x_{\sigma(k)}, x_{\sigma(k+1)} \ldots x_{\sigma(n-1)}\right]\right),
$$

em que $\alpha_{\sigma}^{(k)} \in \mathbb{Z}$. Reescrevendo esta expressão, obtemos

$$
\begin{gathered}
f=\sum_{\sigma \in S_{n-1}}\left(\left(\alpha_{\sigma}^{(0)}+\ldots+\alpha_{\sigma}^{(n-2)}\right) x_{n} x_{\sigma(1)} \ldots x_{\sigma(n-1)}\right. \\
-\alpha_{\sigma}^{(n-2)} x_{\sigma(n-1)} x_{n} x_{\sigma(1)} \ldots x_{\sigma(n-2)}-\alpha_{\sigma}^{(n-3)} x_{\sigma(n-2)} x_{\sigma(n-1)} x_{n} x_{\sigma(1)} \ldots x_{\sigma(n-3)} \\
\left.-\ldots-\alpha_{\sigma}^{(1)} x_{\sigma(2)} \ldots x_{\sigma(n-1)} x_{n} x_{\sigma(1)}-\alpha_{\sigma}^{(0)} x_{\sigma(1)} \ldots x_{\sigma(n-1)} x_{n}\right) .
\end{gathered}
$$

Dessa forma, se $f=0$ segue que $\alpha_{\sigma}^{(0)}=\alpha_{\sigma}^{(1)}=\ldots=\alpha_{\sigma}^{(n-2)}=0, \forall \sigma \in S_{n-1}$ e, portanto, os elementos de $\mathcal{C}$ são linearmente independentes.

Lema 4.23. $L_{n}^{(2)} \cap P_{n-1} x_{n}=\{0\}$. 
Demonstração. Seja $f \in P_{n-1} x_{n}$. Então $f=\sum_{\sigma \in S_{n-1}} \beta_{\sigma} x_{\sigma(1)} \ldots x_{\sigma(n-1)} x_{n}$, em que $\beta_{\sigma} \in \mathbb{Z}$. Suponha que $f \in L_{n}^{(2)}$ e escreva $f$ como na demonstração do lema 4.22, isto é,

$$
f=\sum_{\sigma \in S_{n-1}}\left(\alpha_{\sigma}^{(0)}\left[x_{n}, x_{\sigma(1)} \ldots x_{\sigma(n-1)}\right]+\sum_{k=1}^{n-2} \alpha_{\sigma}^{(k)}\left[x_{n} x_{\sigma(1)} \ldots x_{\sigma(k)}, x_{\sigma(k+1)} \ldots x_{\sigma(n-1)}\right]\right)
$$

em que $\alpha_{\sigma}^{(k)} \in \mathbb{Z}$. Então para cada $\sigma \in S_{n-1}$ temos

$$
\left\{\begin{array}{r}
\alpha_{\sigma}^{(n-2)}=\alpha_{\sigma}^{(n-1)}=\ldots=\alpha_{\sigma}^{(1)}=0 ; \\
\alpha_{\sigma}^{(0)}+\beta_{\sigma}=0 ; \\
\alpha_{\sigma}^{(0)}+\alpha_{\sigma}^{(1)}+\ldots+\alpha_{\sigma}^{(n-2)}=0
\end{array}\right.
$$

e , portanto, $f=0$.

Corolário 4.24. $L_{n}^{(3)} \cap T_{n-1}^{(3)} x_{n}=\{0\}$.

Demonstração. Uma vez que $L_{n}^{(3)} \subset L_{n}^{(2)}$ e $T_{n-1}^{(3)} x_{n} \subset P_{n-1} x_{n}$, o resultado segue do lema 4.23 .

Teorema 4.25. $T_{n}^{(3)}=L_{n}^{(3)} \oplus T_{n-1}^{(3)} x_{n}$.

Demonstração. Uma vez que $\mathcal{A}_{n} \cup \mathcal{B}_{n}$ é uma base para $T_{n}^{(3)}$ (proposição 4.5 ) e que todo elemento $f$ dessa base pode ser escrito na forma $f=f_{1}+f_{2}$, em que $f_{1} \in L_{n}^{(3)}$ e $f_{2} \in T_{n-1}^{(3)} x_{n}$ (lemas 4.11 e 4.21 ), temos que $T_{n}^{(3)}=L_{n}^{(3)}+T_{n-1}^{(3)} x_{n}$. O resultado segue então do corolário 4.24 .

Corolário 4.26. $P_{n} / L_{n}^{(3)}$ é um $\mathbb{Z}$-módulo livre.

Demonstração. Temos que $P_{n}=T_{n}^{(3)} \oplus V_{n}$ (conforme foi observado no final da seção 4.1). Disso e do teorema 4.25 segue que

$$
P_{n}=L_{n}^{(3)} \oplus T_{n-1}^{(3)} x_{n} \oplus V_{n}
$$

Portanto,

$$
P_{n} / L_{n}^{(3)} \cong T_{n-1}^{(3)} x_{n} \oplus V_{n}
$$

Uma vez que $P_{n}$ é um $\mathbb{Z}$-módulo livre, então (pela proposição 2.22 ) $T_{n-1}^{(3)} x_{n} \oplus V_{n}$ é $\mathbb{Z}$-módulo livre, de onde segue o resultado 
Lema 4.27. Sejam $V_{1}$ e $V_{2} \mathbb{Z}$-submódulos de um $\mathbb{Z}$-módulo livre $V$ de modo que $V=$ $V_{1} \oplus V_{2}$. Desta forma, cada elemento $d \in V$ se escreve de maneira única como uma soma $d^{(1)}+d^{(2)}$ em que $d^{(1)} \in V_{1}$ e $d^{(2)} \in V_{2}$. Sejam $\beta_{1}=\left\{a_{i} \mid i \in I\right\}$ e $\beta_{2}=\left\{b_{j} \mid j \in J\right\}$ subconjuntos disjuntos de $V$ tais que $\beta_{1}$ é uma base de $V_{1}$ e $\beta_{1} \cup \beta_{2}$ é uma base de $V$. Então $\left\{b_{j}^{(2)} \mid j \in J\right\}$ é uma base de $V_{2}$.

Demonstração. Seja $b \in V_{2}$. Uma vez que $V_{2} \subset V$, então $b=\sum_{k} \alpha_{k} a_{k}+\sum_{l} \lambda_{l} b_{l}$, em que $\alpha_{k}, \lambda_{l} \in \mathbb{Z}, a_{k} \in \beta_{1}$ e $b_{l} \in \beta_{2}$. Escrevendo $b_{l}=b_{l}^{(1)}+b_{l}^{(2)}$, temos

$$
b=\underbrace{\sum_{k} \alpha_{k} a_{k}+\sum_{l} \lambda_{l} b_{l}^{(1)}}_{c}+\sum_{l} \lambda_{l} b_{l}^{(2)} .
$$

Mas $c \in V_{1} \cap V_{2}=\{0\}$ e, portanto, $b=\sum_{l} \lambda_{l} b_{l}^{(2)}$.

Suponha que $\sum_{t} \lambda_{t} b_{t}^{(2)}=0$, em que $\lambda_{t} \in \mathbb{Z}$ e $b_{t} \in \beta_{2}$. Temos

$$
\sum_{t} \lambda_{t} b_{t}=\sum_{t} \lambda_{t} b_{t}^{(1)}+\sum_{t} \lambda_{t} b_{t}^{(2)}
$$

e, portanto,

$$
\sum_{t} \lambda_{t} b_{t}=\sum_{t} \lambda_{t} b_{t}^{(1)}
$$

Assim $\sum_{t} \lambda_{t} b_{t} \in V_{1}$ e como $\beta_{1}$ é uma base de $V_{1}$, temos

$$
\sum_{t} \lambda_{t} b_{t}=\sum_{r} \alpha_{r} a_{r}
$$

Uma vez que $\beta_{1} \cup \beta_{2}$ é base de $V$, então $\lambda_{t}=0$.

Dado que $T_{n}^{(3)}=L_{n}^{(3)} \oplus T_{n-1}^{(3)} x_{n}$, escreva $d \in T_{n}^{(3)}$ como $d^{(1)}+d^{(2)}$ em que $d^{(1)} \in L_{n}^{(3)}$ e $d^{(2)} \in T_{n-1}^{(3)} x_{n}$. Uma vez que o conjunto $\mathcal{A}_{n-1} x_{n} \cup \mathcal{B}_{n-1} x_{n} \subset \mathcal{A}_{n} \cup \mathcal{B}_{n}$ é uma base de $T_{n-1}^{(3)} x_{n}$, então segue do lema 4.27 que

$$
\mathcal{D}_{n}=\left\{d^{(1)} \mid d \in\left(\mathcal{A}_{n} \backslash \mathcal{A}_{n-1} x_{n}\right) \cup\left(\mathcal{B}_{n} \backslash \mathcal{B}_{n-1} x_{n}\right)\right\}
$$

é uma base de $L_{n}^{(3)}$. 


\subsection{O quociente $H_{m_{1}, m_{2}, \ldots, m_{k-1}, 1} / H_{m_{1}, m_{2}, \ldots, m_{k-1}, 1} \cap L^{(3)}$}

Sejam $m_{1}, \ldots, m_{k}$ inteiros não nulos e seja $H_{m_{1}, \ldots, m_{k}} \mathrm{o} \mathbb{Z}$-módulo gerado pelos polinômios de $\mathbb{Z}\langle X\rangle$ nas variáveis $x_{1}, \ldots, x_{k}$ com multigrau $\left(m_{1}, \ldots, m_{k}\right)$. Nesta seção, será considerado o caso em que $m_{k}=1$.

Seja $n=m_{1}+\ldots+m_{k-1}+1$ e considere a aplicação $\phi_{m_{1}, \ldots, m_{k-1}, 1}$ de $X$ em $\mathbb{Z}\langle X\rangle$ tal que

$$
\left\{\begin{array}{l}
x_{i} \mapsto x_{1}, \quad i=1, \ldots, m_{1} \\
x_{i} \mapsto x_{2}, \quad i=m_{1}+1, \ldots, m_{1}+m_{2} \\
x_{i} \mapsto x_{3}, \quad i=m_{1}+m_{2}+1, \ldots, m_{1}+m_{2}+m_{3} \\
\ldots \ldots \ldots \ldots \ldots \ldots \ldots \ldots \ldots \\
x_{i} \mapsto x_{k-1}, \quad i=m_{1}+m_{2}+\ldots+m_{k-2}+1, \ldots, m_{1}+m_{2}+\ldots+m_{k-2}+m_{k-1} \\
x_{n} \mapsto x_{k} .
\end{array}\right.
$$

Seja $\Phi_{m_{1}, \ldots, m_{k-1}, 1}$ o endomorfismo de $\mathbb{Z}\langle X\rangle$ que extende $\phi_{m_{1}, \ldots, m_{k-1}, 1}$. Denote $\Phi=$ $\Phi_{m_{1}, \ldots, m_{k-1}, 1}$.

Exemplo 4.28. Seja $f\left(x_{1}, x_{2}, x_{3}, x_{4}\right)=x_{1} x_{2} x_{1} x_{2} x_{3} x_{2} x_{3} x_{4} x_{3} \in H_{2,3,3,1}$. Então $f=$ $\Phi_{2,3,3,1}(g)$ para todo $g \in P_{9}$ da forma

$$
g\left(x_{1}, x_{2}, \ldots, x_{9}\right)=x_{i_{1}} x_{j_{1}} x_{i_{2}} x_{j_{2}} x_{k_{1}} x_{j_{3}} x_{k_{2}} x_{9} x_{k_{3}}
$$

em que $\left\{i_{1}, i_{2}\right\}=\{1,2\},\left\{j_{1}, j_{2}, j_{3}\right\}=\{3,4,5\}$ e $\left\{k_{1}, k_{2}, k_{3}\right\}=\{6,7,8\}$.

De um modo geral, dado um monômio $f \in H_{m_{1}, \ldots, m_{k-1}, 1}$, existem $m_{1} ! m_{2} ! \cdots m_{k-1}$ ! monômios $g \in P_{n}$ tais que $\Phi_{m_{1}, \ldots, m_{k-1}, 1}(g)=f$. Desta forma,

$$
\Phi_{m_{1}, \ldots, m_{k-1}, 1}\left(P_{n}\right)=H_{m_{1}, \ldots, m_{k-1}, 1}
$$

Temos também que

$$
\begin{aligned}
& \text { - } \Phi_{m_{1}, \ldots, m_{k-1}, 1}\left(L_{n}^{(3)}\right)=L^{(3)} \cap H_{m_{1}, \ldots, m_{k-1}, 1} ; \\
& \text { - } \Phi_{m_{1}, \ldots, m_{k-1}, 1}\left(T_{n}^{(3)}\right)=T^{(3)} \cap H_{m_{1}, \ldots, m_{k-1}, 1} ; \\
& \text { - } \Phi\left(T_{n-1}^{(3)} x_{n}\right)=\left(T^{(3)} \cap H_{m_{1}, \ldots, m_{k-1}}\right) x_{k} .
\end{aligned}
$$


Lema 4.29. Seja $f \in P_{m}$ e $M$ o $\mathbb{Z}$-módulo de $\mathbb{Z}\langle X\rangle$ gerado pelo conjunto

$$
\left\{g_{0} f\left(g_{1}, \ldots, g_{m}\right) g_{m+1} \mid g_{0}, g_{1}, \ldots, g_{m}, g_{m+1} \in \mathbb{Z}\langle X\rangle\right\}
$$

Seja $h \in \mathbb{Z}\langle X\rangle$. Se $h$ está em $M$, então as componentes multi-homogêneas de $h$ também estão em $M$

Demonstração. Escreva $f\left(x_{1}, \ldots, x_{m}\right)=\sum_{\sigma \in S_{m}} \alpha_{\sigma} x_{\sigma(1)} \ldots x_{\sigma(m)}$ em que $\alpha_{\sigma} \in \mathbb{Z}$ e sejam $g_{0}, g_{1}, \ldots, g_{m+1} \in \mathbb{Z}\langle X\rangle$. Escreva $g_{j}=\sum_{a_{j}} g_{j}^{\left(a_{j}\right)}, j=0,1, \ldots, m+1$, em que $g_{j}^{\left(a_{j}\right)}$ é a componente multi-homogênea de $g_{j}$ de multigrau $a_{j}=\left(a_{j_{1}}, \ldots, a_{j_{t}}\right)$. Temos

$$
\begin{gathered}
g_{0} f\left(g_{1}, \ldots, g_{m}\right) g_{m+1}=\sum_{\sigma \in S_{m}} \alpha_{\sigma} g_{0} g_{\sigma(1)} \ldots g_{\sigma(m)} g_{m+1} \\
=\sum_{\sigma \in S_{m}} \alpha_{\sigma}\left(\left(\sum_{a_{0}} g_{0}^{\left(a_{0}\right)}\right)\left(\sum_{a_{\sigma(1)}} g_{\sigma(1)}^{\left(a_{\sigma(1)}\right)}\right) \ldots\left(\sum_{a_{\sigma(m)}} g_{\sigma(m)}^{\left(a_{\sigma(m)}\right)}\right)\left(\sum_{a_{m+1}} g_{m+1}^{\left(a_{m+1}\right)}\right)\right) \\
=\sum_{\sigma \in S_{m}} \alpha_{\sigma}\left(\sum_{a_{0}, a_{1}, \ldots, a_{m}, a_{m+1}} g_{0}^{\left(a_{0}\right)} g_{\sigma(1)}^{\left(a_{\sigma(1)}\right)} \ldots g_{\sigma(m)}^{\left(a_{\sigma(m)}\right)} g_{m+1}^{\left(a_{m+1}\right)}\right) \\
=\sum_{a_{0}, a_{1}, \ldots, a_{m}, a_{m+1}} \sum_{\sigma \in S_{m}} \alpha_{\sigma} g_{0}^{\left(a_{0}\right)} g_{\sigma(1)}^{\left(a_{\sigma(1)}\right)} \ldots g_{\sigma(m)}^{\left(a_{\sigma(m)}\right)} g_{m+1}^{\left(a_{m+1}\right)} \\
=\sum_{a_{0}, a_{1}, \ldots, a_{m}, a_{m+1}} g_{0}^{\left(a_{0}\right)} f\left(g_{1}^{\left(a_{1}\right)}, \ldots, g_{m}^{\left(a_{m}\right)}\right) g_{m+1}^{\left(a_{m+1}\right)}
\end{gathered}
$$

Note que $g_{0}^{\left(a_{0}\right)} f\left(g_{1}^{\left(a_{1}\right)}, \ldots, g_{m}^{\left(a_{m}\right)}\right) g_{m+1}^{\left(a_{m+1}\right)}$ é um elemento de $M$ que é multi-homogêneo de multigrau $a_{0}+a_{1}+\ldots+a_{m+1}$.

Suponha que $h=g_{0} f\left(g_{1}, \ldots, g_{m}\right) g_{m+1}$ e seja $h^{(a)}$ a componente multi-homogênea de $h$ de multigrau $a$. Então

$$
h^{(a)}=\sum_{a_{0}+a_{1}+\ldots+a_{m+1}=a} g_{0}^{\left(a_{0}\right)} f\left(g_{1}^{\left(a_{1}\right)}, \ldots, g_{m}^{\left(a_{m}\right)}\right) g_{m+1}^{\left(a_{m+1}\right)}
$$

e, portanto, $h^{(a)} \in M$.

No caso em que $h$ é uma combinação linear de elementos da forma $g_{0} f\left(g_{1}, \ldots, g_{m}\right) g_{m+1}$, então uma componente multi-homogênea $h^{(a)}$ de multigrau $a$ de $h$ é a soma das componentes multi-homogêneas de multigrau $a$ das parcelas dessa combinação que possuem essa componente. Portanto, $h^{(a)} \in M$. 
Corolário 4.30. Seja $h \in \mathbb{Z}\langle X\rangle$ tal que $h^{(a)}$ é a componente multi-homogênea de $h$ de multigrau $a$.

i. Se $h \in L^{(3)}$ então $h^{(a)} \in L^{(3)}$;

ii. Se $h \in T^{(3)}$ ent $\tilde{a} o h^{(a)} \in T^{(3)}$.

Demonstração. Basta notar que $L^{(3)}$ é o $\mathbb{Z}$-módulo de $\mathbb{Z}\langle X\rangle$ gerado pelo conjunto

$$
\left\{f\left(g_{1}, g_{2}, g_{3}\right) \mid g_{1}, g_{2}, g_{3} \in \mathbb{Z}\langle X\rangle\right\}
$$

e $T^{(3)}$ é o $\mathbb{Z}$-módulo de $\mathbb{Z}\langle X\rangle$ gerando pelo conjunto

$$
\left\{g_{0} f\left(g_{1}, g_{2}, g_{3}\right) g_{4} \mid g_{0}, g_{1}, g_{2}, g_{3}, g_{4} \in \mathbb{Z}\langle X\rangle\right\}
$$

em que $f=\left[x_{1}, x_{2}, x_{3}\right]$.

Lema 4.31. $\left(H_{m_{1}, \ldots, m_{k-1}, 1} \cap L^{(3)}\right) \cap\left(\left(H_{m_{1}, \ldots, m_{k-1}} \cap T^{(3)}\right) x_{k}\right)=\{0\}$.

Demonstração. Seja $f=f\left(x_{1}, \ldots, x_{k-1}, x_{k}\right) \in H_{m_{1}, \ldots, m_{k-1}, 1}$. Seja

$$
\begin{gathered}
g\left(x_{1}, \ldots, x_{m_{1}}, x_{m_{1}+1}, \ldots, x_{m_{1}+m_{2}}, \ldots, x_{m_{1}+\ldots+m_{k-2}+1}, \ldots, x_{m_{1}+\ldots+m_{k-2}+m_{k-1}}, x_{n}\right) \\
=f\left(x_{1}+\ldots+x_{m_{1}}, x_{m_{1}+1}+\ldots+x_{m_{1}+m_{2}}, \ldots, x_{m_{1}+\ldots+m_{k-2}+1}+\ldots+x_{m_{1}+\ldots+m_{k-2}+m_{k-1}}, x_{n}\right)
\end{gathered}
$$

em que $n=m_{1}+\ldots+m_{k-1}+1$, a linearização completa de $f$. Seja

$$
h\left(x_{1}, \ldots, x_{m_{1}}, x_{m_{1}+1}, \ldots, x_{m_{1}+m_{2}}, \ldots, x_{m_{1}+\ldots+m_{k-2}+1}, \ldots, x_{m_{1}+\ldots+m_{k-2}+m_{k-1}}, x_{n}\right)
$$

a componente multilinear de $g$.

Suponha que

$$
f \in\left(H_{m_{1}, \ldots, m_{k-1}, 1} \cap L^{(3)}\right) \cap\left(\left(H_{m_{1}, \ldots, m_{k-1}} \cap T^{(3)}\right) x_{k}\right) .
$$

Então $g \in L^{(3)} \cap T^{(3)} x_{n}$. Uma vez que $h \in P_{n}$, segue do corolário 4.30 que $h \in L_{n}^{(3)} \cap T_{n-1}^{(3)} x_{n}$. Mas pelo corolário 4.24 temos que $h=0$.

Por outro lado,

$$
h(\underbrace{x_{1}, \ldots, x_{1}}_{m_{1}}, \underbrace{x_{2}, \ldots, x_{2}}_{m_{2}}, \ldots, \underbrace{x_{k-1}, \ldots, x_{k-1}}_{m_{k-1}}, x_{k})=m_{1} ! \cdots m_{k-1} ! f\left(x_{1}, \ldots, x_{k-1}, x_{k}\right)
$$

e, portanto, $m_{1} ! \cdots m_{k-1} ! f\left(x_{1}, \ldots, x_{k-1}, x_{k}\right)=0$. Mas $\mathbb{Z}\langle X\rangle$ é um $\mathbb{Z}$-módulo livre e, portanto, $f\left(x_{1}, \ldots, x_{k}, x_{k+1}\right)=0$. 
Corolário 4.32. $H_{m_{1}, \ldots, m_{k-1}, 1} \cap T^{(3)}=\left(H_{m_{1}, \ldots, m_{k-1}, 1} \cap L^{(3)}\right) \oplus\left(\left(H_{m_{1}, \ldots, m_{k-1}} \cap T^{3}\right) x_{k}\right)$

Demonstração. Seja $n=m_{1}+\cdots+m_{k-1}+1$. Uma vez que $T_{n}^{(3)}=L_{n}^{(3)} \oplus T_{n-1}^{(3)} x_{n}$, então aplicando $\Phi_{m_{1}, \ldots, m_{k-1}, 1}$ em ambos os lados dessa expressão e considerando o lema 4.31 temos o resultado.

Para a continuidade do texto, lembramos alguns resultados e definições dados anteriormente. No final da seção 4.1 vimos que

$$
P_{n}=T_{n}^{(3)} \oplus V_{n}
$$

em que $V_{n}$ é o $\mathbb{Z}$-módulo gerado pelo conjunto

$$
\begin{gathered}
\mathcal{R}_{n}^{\prime}=\left\{\left[x_{i_{1}}, x_{i_{2}}\right] \ldots\left[x_{i_{2 t-1}}, x_{i_{2 t}}\right] x_{j_{1}} \ldots x_{j_{n-2 t}} \mid 0 \leq 2 t \leq n\right. \\
\left.i_{1}<\ldots<i_{2 t}, j_{1}<\ldots<j_{n-2 t},\left\{i_{1} \ldots i_{2 t}, j_{1}, \ldots, j_{n-2 t}\right\}=\{1, \ldots, n\}\right\} .
\end{gathered}
$$

Além disso, no capítulo 1 (conceitos preliminares), definimos o conjunto $\mathcal{S}$ formado pelos elementos da forma

$$
\left[x_{i_{1}}, x_{i_{2}}\right] \cdots\left[x_{i_{2 t-1}}, x_{i_{2 t}}\right] x_{1}^{b_{1}} \ldots x_{k}^{b_{k}}
$$

em que

- $b_{j} \in\left\{m_{j}, m_{j}-1\right\}, j=1, \ldots, k$;

- $i_{1}<i_{2}<\ldots<i_{2 t}, \quad 0 \leq 2 t \leq k$, são os inteiros $j \in\{1,2, \ldots, k\}$ para os quais $b_{j}=m_{j}-1$,

e vimos que

$$
\left\{f+T^{(3)} \mid f \in \mathcal{S}\right\}
$$

é um conjunto linearmente independente no $\mathbb{Z}$-módulo $H_{m_{1}, \ldots, m_{k}} /\left(T^{(3)} \cap H_{m_{1}, \ldots, m_{k}}\right)$. Desta forma, denotando por $\tilde{\mathcal{S}}$ o $\mathbb{Z}$-módulo gerado pelo conjunto $\mathcal{S}$, temos que $T^{(3)} \cap \tilde{\mathcal{S}}=\{0\}$.

Lema 4.33. Se $f \in \mathcal{R}_{n}^{\prime}$ então $\Phi_{m_{1}, \ldots, m_{k-1}, 1}(f) \in\left(H_{m_{1}, \ldots, m_{k-1}, 1} \cap T^{(3)}\right) \cup \mathcal{S}$.

Demonstração. Seja $f \in \mathcal{R}_{n}^{\prime}$. Temos que $f=g h$ em que

1) $h=\left(x_{1}^{a_{1}} \ldots x_{m_{1}}^{a_{m_{1}}}\right)\left(x_{m_{1}+1}^{a_{m_{1}+1}} \ldots x_{m_{1}+m_{2}}^{a_{m_{1}+m_{2}}}\right) \ldots\left(x_{m_{1}+\ldots+m_{k-2}+1}^{a_{m_{1}+\ldots+m_{k-2}+1}} \ldots x_{m_{1}+\ldots+m_{k-1}}^{a_{m_{1}+\ldots+m_{k-1}}}\right) x_{n}^{a_{n}}, a_{j} \in$ $\{0,1\}$ para todo $j=1, \ldots, n$; 
2) g é um produto de comutadores da forma

$$
\left[x_{i_{1}}, x_{i_{2}}\right] \ldots\left[x_{i_{2 t-1}}, x_{i_{2 t}}\right]
$$

em que $\left\{x_{i_{1}}, x_{i_{2}}, \ldots, x_{i_{2 t}}\right\}$ é o subconjunto das variáveis $\left\{x_{1}, \ldots, x_{n}\right\}$ que tem expoente 0 em $h$ e dispostas de forma que $i_{1}<i_{2}<\ldots<i_{2 t}$.

Denote $\Phi=\Phi_{m_{1}, \ldots, m_{k-1}, 1}$. Temos

$$
\Phi(f)=x_{1}^{\ell_{1}} x_{2}^{\ell_{2}} \ldots x_{k-1}^{\ell_{k-1}} x_{k}^{a_{n}}\left[\Phi\left(x_{i_{1}}\right), \Phi\left(x_{i_{2}}\right)\right] \ldots\left[\Phi\left(x_{i_{2 t-1}}\right), \Phi\left(x_{i_{2 t}}\right)\right]
$$

em que $0 \leq \ell_{j} \leq m_{j}$ para todo $j=1, \ldots, k-1$.

Se $\ell_{j} \leq m_{j}-2$ para algum $j \in\{1, \ldots, k-1\}$, então $\Phi\left(x_{i_{r}}\right)=\Phi\left(x_{i_{s}}\right)$ para, pelo menos, um par $r, s \in\{1, \ldots, 2 t\}$, com $r \neq s$. Pelo corolário 2.54 temos que, nesse caso, $\Phi(f) \in T^{(3)}$. Se $\ell_{j} \in\left\{m_{j}-1, m_{j}\right\}$ para todo $j=1, \ldots, k-1$, então $\Phi(f) \in \mathcal{S}$.

Defina $V_{m_{1}, \ldots, m_{k-1}, 1}:=\Phi_{m_{1}, \ldots, m_{k-1}, 1}\left(V_{n}\right), n=m_{1}+\ldots+m_{k-1}+1$.

Corolário 4.34. $V_{m_{1}, \ldots, m_{k-1}, 1} \subset\left(H_{m_{1}, \ldots, m_{k-1}, 1} \cap T^{(3)}\right) \bigoplus \tilde{\mathcal{S}}$.

Demonstração. Seja $f \in V_{m_{1}, \ldots, m_{k-1}, 1}$. Então $f=\Phi_{m_{1}, \ldots, m_{k-1}, 1}(g)$ para algum $g \in V_{n}$. Logo,

$$
f=\sum_{i} \alpha_{i} \Phi_{m_{1}, \ldots, m_{k-1}, 1}\left(g_{i}\right)
$$

em que $\alpha_{i} \in \mathbb{Z}$ e $g_{i} \in \mathcal{R}_{n}^{\prime}$. Então o resultado segue da proposição 4.33 e do fato que $T^{(3)} \cap \tilde{\mathcal{S}}=\{0\}$.

O teorema abaixo equivale ao teorema 1.12 enunciado no capítulo 1 (introdução).

Teorema 4.35. $H_{m_{1}, \ldots, m_{k-1}, 1} / H_{m_{1}, \ldots, m_{k-1}, 1} \cap L^{(3)}$ é um $\mathbb{Z}$-módulo livre.

Demonstração. Como antes, tome $n=m_{1}+\ldots+m_{k-1}$. Tendo em vista que $P_{n}=T_{n}^{(3)} \oplus V_{n}$, então aplicando $\Phi_{m_{1}, \ldots, m_{k-1}, 1} \mathrm{em}$ ambos os lados dessa expressão, temos

$$
H_{m_{1}, \ldots, m_{k-1}, 1}=\left(H_{m_{1}, \ldots, m_{k-1}, 1} \cap T^{(3)}\right)+V_{m_{1}, \ldots, m_{k-1}, 1} .
$$

Segue então, do corolário 4.34, que

$$
H_{m_{1}, \ldots, m_{k-1}, 1}=\left(H_{m_{1}, \ldots, m_{k-1}, 1} \cap T^{(3)}\right) \oplus \tilde{\mathcal{S}}
$$


Mas, pelo corolário 4.32, temos

$$
H_{m_{1}, \ldots, m_{k-1}, 1} \cap T^{(3)}=\left(H_{m_{1}, \ldots, m_{k-1}, 1} \cap L^{(3)}\right) \oplus\left(\left(H_{m_{1}, \ldots, m_{k-1}} \cap T^{3}\right) x_{k}\right)
$$

e, portanto

$$
H_{m_{1}, \ldots, m_{k-1}, 1}=\left(H_{m_{1}, \ldots, m_{k-1}, 1} \cap L^{(3)}\right) \oplus\left(\left(H_{m_{1}, \ldots, m_{k-1}} \cap T^{3}\right) x_{k}\right) \oplus \tilde{\mathcal{S}}
$$

De onde segue que

$$
H_{m_{1}, \ldots, m_{k-1}, 1} / H_{m_{1}, \ldots, m_{k-1}, 1} \cap L^{(3)} \cong\left(\left(H_{m_{1}, \ldots, m_{k-1}} \cap T^{3}\right) x_{k}\right) \oplus \tilde{\mathcal{S}} .
$$

Uma vez que todo submódulo de $\mathbb{Z}\langle X\rangle$ é livre (proposição 2.22), segue o resultado.

Corolário 4.36. Seja $f=f\left(x_{1}, \ldots, x_{k}\right) \in H_{m_{1}, \ldots, m_{k-1}, 1}$ e $r$ um inteiro não nulo. Se $r f \in L^{(3)}$, então $f \in L^{(3)}$.

Demonstração. Segue do teorema 4.35 que o $\mathbb{Z}$-módulo $H_{m_{1}, \ldots, m_{k-1}, 1} / H_{m_{1}, \ldots, m_{k-1}, 1} \cap L^{(3)}$ é livre de torção. Portanto, se $r f+L^{(3)}=0$, então $f+L^{(3)}=0$, de onde segue o resultado.

\subsection{Os elementos do centro de $B$ contidos em $B_{1}$}

Lembramos que a série central inferior de $\mathbb{Z}\langle X\rangle$ vista como álgebra de Lie é a série dos ideais de Lie $L^{(i)} \subset \mathbb{Z}\langle X\rangle$ definidos recursivamente por $L^{(1)}=\mathbb{Z}\langle X\rangle$ e $L^{(i+1)}=\left[L^{(i)}, \mathbb{Z}\langle X\rangle\right]$ $(i \geq 1)$. Além disso, $B:=\bigoplus_{i \geq 1} B_{i}\left(B_{i}=L^{(i)} / L^{(i+1)}\right)$ é a correspondente álgebra de Lie graduada associada a série central inferior de $\mathbb{Z}\langle X\rangle^{(-)}$. Note que, visto como $\mathbb{Z}$-submódulo de $\mathbb{Z}\langle X\rangle, L^{(i)}(i \geq 2)$ é gerado pelo conjunto $\left\{\left[f_{1}, \ldots, f_{i}\right] \mid f_{i} \in \mathbb{Z}\langle X\rangle\right\}$. Lembramos ainda que $T^{(i)}$ é o ideal bilateral em $\mathbb{Z}\langle X\rangle$ gerado como ideal pelos elementos $\left[f_{1}, \ldots, f_{i}\right]$ $\left(f_{1}, \ldots, f_{i} \in \mathbb{Z}\langle X\rangle\right)$.

Uma observação para a sequência do texto é a de que o $\mathbb{Z}$-módulo $L^{(i)}, i \geq 1$, tem a seguinte propriedade:

$f\left(x_{1}, \ldots, x_{k}\right) \in L^{(i)}$ se, e somente se, $f\left(g_{1}, \ldots, g_{k}\right) \in L^{(i)}$ para todos $g_{1}, \ldots, g_{k} \in \mathbb{Z}\langle X\rangle$.

Um módulo com essa propriedade é dito um $T$-módulo. Outra observação é que

$$
T^{(i)}=L^{(i)} \cdot \mathbb{Z}\langle X\rangle \quad(i \geq 2)
$$


em que $L^{(i)} \cdot \mathbb{Z}\langle X\rangle$ é o $\mathbb{Z}$-módulo gerado pelos elementos da forma $g h$ em que $g \in L^{(i)}$ e $h \in \mathbb{Z}\langle X\rangle$. Basta ver que

$$
f_{0}\left[f_{1}, \ldots, f_{i}\right] f_{i+1}=\left[f_{1}, \ldots, f_{i}\right] f_{0} f_{i+1}-\left[f_{1}, \ldots, f_{i}, f_{0}\right] f_{i+1}
$$

em que $f_{0}, f_{1}, \ldots, f_{i+1} \in \mathbb{Z}\langle X\rangle$.

Na demonstração do lema abaixo será utilizado o corolário 2.64 (dado no capítulo 2) que afirma que dado $f \in \mathbb{Z}\langle X\rangle$, então

$$
f+L^{(2)} \in Z(B) \text { se, e somente se, }[f, g] \in L^{(3)} \text { para todo } g \in \mathbb{Z}\langle X\rangle .
$$

Lema 4.37. $\left(L^{(2)}+T^{(3)}\right) / L^{(2)} \subset Z(B)$.

Demonstração. Seja $f=g+h$, em que $g \in L^{(2)}$ e $h \in T^{(3)}$, e seja $a \in \mathbb{Z}\langle X\rangle$. Claramente $[g, a] \in L^{(3)}$. O lema 4.9 afirma que $\left[T^{(3)}, \mathbb{Z}\langle X\rangle\right] \subset L^{(3)}$ e, portanto, $[h, a] \in L^{(3)}$. Desta forma, $[f, a] \in L^{(3)}$. Assim, pelo corolário 2.64, temos que $f+L^{(2)} \in Z(B)$.

Observamos que a demonstração original do lema acima se deve a Feigin e Shoikhetem (veja [17]).

Lema 4.38. Seja $f=f\left(x_{1}, \ldots, x_{k}\right) \in \mathbb{Z}\langle X\rangle$. Então

$$
f+L^{(2)} \in Z(B) \text { se, e somente se, }\left[f\left(x_{1}, \ldots, x_{k}\right), x_{k+1}\right] \in L^{(3)} .
$$

Demonstração. Segue do corolário 2.64 e do fato de que $L^{(3)}$ é um $T$-módulo.

Lema 4.39. Seja $r$ um inteiro não nulo e $f \in \mathbb{Z}\langle X\rangle$. Se $r f+L^{(2)} \in Z(B)$, então $f+L^{(2)} \in Z(B)$.

Demonstração. Suponha que $f=f\left(x_{1}, \ldots, x_{k}\right)$. Uma vez que $r f+L^{(2)} \in Z(B)$, segue do lema 4.38 que $r\left[f, x_{k+1}\right] \in L^{(3)}$. Suponha que $f=f\left(x_{1}, \ldots, x_{k}\right) \in H_{m_{1}, \ldots, m_{k}}$. Então, $r\left[f, x_{k+1}\right] \in H_{m_{1}, \ldots, m_{k}, 1} \cap L^{(3)}$. Portanto, pelo corolário 4.36, $\left[f, x_{k+1}\right] \in L^{(3)}$. Novamente pelo lema 4.38, temos que $f+L^{(2)} \in Z(B)$.

No caso geral em que $f$ não é necessariamente multi-homogêneo, escreva

$$
f=\sum_{m=\left(m_{1}, \ldots, m_{k}\right)} f^{(m)}
$$


em que $f^{(m)}$ é a componente multi-homogênea de $f$ multigrau $m=\left(m_{1}, \ldots, m_{k}\right)$. Assim, $\sum_{m} r\left[f^{(m)}, x_{k+1}\right] \in L^{(3)}$. Mas, pelo corolário 4.30, temos que $r\left[f^{(m)}, x_{k+1}\right] \in L^{(3)} \mathrm{e}$, desta forma, o resultado segue do caso anterior.

Conforme foi definido no início deste capítulo, $\bar{B}_{1}:=B_{1} / \mathcal{J}$ em que $\mathcal{J}$ é a imagem de $T^{(3)}$ em $B_{1}$. Claramente, $\mathcal{J} \cong\left(L^{(2)}+T^{(3)}\right) / L^{(2)}$. O lema abaixo corresponde a proposição 3.10 de $[8]$.

Lema 4.40. A torção em $\bar{B}_{1}\left(\mathbb{Z}\left\langle x_{1}, \ldots, x_{n}\right\rangle\right)\left(m_{1}, \ldots, m_{n}\right)$, em que $\left(m_{1}, \ldots, m_{n}\right)$ denota a parte de multigrau $m_{1}, \ldots, m_{n}$, é isomorfa a $\left(\mathbb{Z} / m d c\left(m_{1}, \ldots, m_{n}\right)\right)^{2^{n-2}}$.

Desta forma, $\operatorname{Tor}\left(\bar{B}_{1}\right) \neq\{0\}$. Conforme definimos anteriormente, dado um $\mathbb{K}$-módulo $M$ ( $\mathbb{K}$ domínio) e um $\mathbb{K}$-submódulo $N$ de $M$, então o isolador de $N$ é definido da seguinte forma

$$
I(N)=\{m \in M \mid \exists k \in \mathbb{K}, k \neq 0, \text { tal que } k m \in N\} .
$$

Claramente, $N \subset M$. Segue diretamente da definição que $\operatorname{Tor}(M / N) \neq\{0\}$ se, e somente se, $N \varsubsetneqq I(N)$. Assim, $\mathcal{J} \varsubsetneqq I(\mathcal{J})$. Em outras palavras, o isolador de $\mathcal{J}$ é maior que $\mathcal{J}$.

O teorema abaixo é o resultado principal desta seção e equivale ao teorema 1.11 enunciado no capítulo 1 (introdução).

Teorema 4.41. $I(\mathcal{J}) \subset Z(B)$.

Demonstração. Seja $f \in \mathbb{Z}\langle X\rangle$ tal que $f+L^{(2)} \in I(\mathcal{J})$. Logo, existe um inteiro não nulo $r$ tal que $r f+L^{(2)} \in \mathcal{J} \subset Z(B)$. Pelo lema 4.39, temos que $f+L^{(2)} \in Z(B)$.

Desta forma, definimos a álgebra de Lie graduada

$$
\widetilde{B}:=B / I(\mathcal{J})=B_{1} / I(\mathcal{J}) \oplus B_{2}(A) \oplus B_{2}(A) \oplus \ldots
$$




\section{Referências Bibliográficas}

[1] N. Abughazalah, P. Etingof, On properties of the lower central series of associative algebras, arXiv:1508.00943

[2] B. Amberg, Ya. Sysak, Associative rings whose adjoint semigroup is locally nilpotent, Archiv der Mathematik (Basel) 76 (2001), 426-435.

[3] N. Arbesfeld, D. Jordan, New results on the lower central series quotients of a free associative algebra, Journal of Algebra 323 (2010), 1813-1825. arXiv:0902.4899

[4] M. Balagović, A. Balasubramanian, On the lower central series quotients of a graded associative algebra, Journal of Algebra 328 (2011), 287-300. arXiv:1004.3735

[5] Bakhturin, Yu. A. Identical relations in Lie algebras. VNU Science Press, Utrecht, (1987).

[6] A. Bapat, D. Jordan, Lower central series of free algebras in symmetric tensor categories, Journal of Algebra 373 (2013), 299-311. arXiv:1001.1375

[7] C. Bekh-Ochir and D. M. Riley, On the Grassmann T-space, Journal of Algebra and its Applications, 7 (2008), 319-336.

[8] S. Bhupatiraju, P. Etingof, D. Jordan, W. Kuszmaul, J. Li, Lower central series of a free associative algebra over the integers and finite fields, Journal of Algebra 372 (2012), 251-274. arXiv:1203.1893

[9] N. Bourbaki, Elements of Mathematics: Lie Groups and Lie Algebra, Chapters 1-3, Springer-Verlag, New York, (1989). 
[10] K. Cordwell, T. Fei, K. Zhou, On lower central series quotients of finitely generated algebras over $\mathbb{Z}$, Journal of Algebra 423 (2015), 559-572. arXiv:1309.1237

[11] E.A. Costa, A. Krasilnikov, Relations in universal Lie nilpotente associative algebras of class 4 (www.arxiv.org/1306.4294v2)

[12] G. Deryabina, A. Krasilnikov, The torsion subgroup of the additive group of a Lie nilpotent associative ring of class 3, Journal of Algebra 428 (2015), 230-255. (www.arxiv.org/1308.4172)

[13] G. Dobrovolska, J. Kim, and X. Ma, On the lower central series of an associative algebra: (with an appendix by Pavel Etingof), Journal of Algebra, 320(1) (2008), 213-237.

[14] G. Dobrovolska, P. Etingof, An upper bound for the lower central series quotients of a free associative algebra, International Mathematics Research Notices (2008), no. 12, Art. ID rnn039, 10 pp. arXiv:0801.1997

[15] V. Drensky, Free algebras and PI-algebras, Graduate Course in Algebra, Springer, Singapore (1999).

[16] P. Etingof, J. Kim, X. Ma, On universal Lie nilpotent associative algebras, Journal of Algebra 321 (2009), 697-703, arXiv:0805.1909.

[17] B. Feigin, B. Shoikhet, On $[A, A] /[A,[A, A]]$ and on a $W_{n}-$ action on the consecutive commutators of free associative algebra, Mathematical Research Letters 14 (2007), 781-795. arXiv:math/0610410.

[18] A. Garcia, Y. Lequain, Elementos de Álgebra, 5.e.d Rio de Janeiro: IMPA, (2008).

[19] A. Giambruno, P. Koshlukov, On the identities of the Grassmann algebra in characteristic $p>0$, Israel Journal of Mathematics 122 (2001), 305-316.

[20] A. Giambruno, M. Zaicev, Polynomial Identities and Asymptotic Methods, Mathematical Surveys and Monographs, 122, American Mathematical Society, Providence, RI, (2015). 
[21] A.S. Gordienko, Codimensions of commutators of length 4, Russian Mathematical Surveys 62 (2007), 187-188.

[22] A. V. Grishin, S. V. Pchelintsev, On centres of relatively free associative algebras with a Lie nilpotency identity, Mat. Sb., 206:11 (2015), 113-130

[23] C.K. Gupta, A.N. Krasil'nikov, A solution of a problem of Plotkin an Vovsi and an application to varieties of groups, Journal of the Australian Mathematical Society (Series A) 67 (1999), 329-355.

[24] N. Gupta, F. Levin, On the Lie ideals of a ring, Journal of Algebra 81 (1983), $225-231$.

[25] D. Jordan, H. Orem, An algebro-geometric construction of lower central series of associative algebras, International Mathematics Research Notices (2015) No. 15, 63306352. arXiv:1302.3992

[26] S. A. Jennings, On rings whose associated Lie rings are nilpotent, Bulletin of the American Mathematical Society 53 (1947), 593-597.

[27] G. Kerchev, On the filtration of a free algebra by its associative lower central series, Journal of Algebra 375 (2013), 322-327. arXiv:1101.5741

[28] E. I. Khukhro, Nilpotent groups and their automorphisms, De Gruyter, Berlin, (1993).

[29] A.N. Krasil'nikov, On the semigroup nilpotency and the Lie nilpotency of associative algebras, Mathematical Notes 62 (1997), 426-433.

[30] V.N. Latysev, On the choise of basis in a T-ideal, Sibirskii Matematicheskii Zhurnal (Siberian Mathematical Journal) 4 (1963), 1122-1127. (in Russian)

[31] V.N. Latysev, On finite generation of a T-ideal with the element $\left[x_{1}, x_{2}, x_{3}, x_{4}\right]$, Sibirskii Matematicheskii Zhurnal (Siberian Mathematical Journal) 6 (1965), 14321434. (in Russian) 
[32] A. P. Popov, Some finitely based varieties of rings, C. R. Academie Bulgare Sciences 32 (1979), 855-858.

[33] D.M. Riley, V. Tasić, Mal'cev nilpotent algebras, Archiv der Mathematik (Basel) 72 (1999), 22-27.

[34] W. Specht, Gesetze in Ringen, I, Math. Z. 52(5) (1950) 557-589.

[35] Ya. P. Sysak, The adjoint group of radical rings and related questions, Ischia Group Theory 2010: Proceedings of the Conference. Edited by Patrizia Longobardi, Mariagrazia Bianchi, Mercede Maj, 344-365.

[36] I.B. Volichenko, The T-ideal generated by the element $\left[x_{1}, x_{2}, x_{3}, x_{4}\right]$, Preprint 22 (1978), Institute of Mathematics of the Academy os Sciences of the Belorussian SSR. (in Russian). 